\title{
Thiophene-fused BODIPY Dimers and Tetramers from Oxidative Aromatic Couplings as Near-Infrared Dyes
}

Qingbao Gong, ${ }^{\text {a }}$ Qinghua $\mathrm{Wu},{ }^{\mathrm{a}, \mathrm{b}}$ Xing Guo, ${ }^{\mathrm{a}} \mathrm{Heng} \mathrm{Li},{ }^{\mathrm{a}}$ Wanwan $\mathrm{Li},{ }^{\mathrm{a}}$ Changjiang $\mathrm{Yu},{ }^{\mathrm{a}}$ Erhong $\mathrm{Hao}^{\mathrm{a}, *}$ and Lijuan $\mathrm{Jiao}^{\mathrm{a}, *}$

${ }^{a}$ Key Laboratory of Functional Molecular Solids, Ministry of Education; School of Chemistry and Materials Science, Anhui Normal University, Wuhu, 241002, China.

${ }^{b}$ School of Pharmaceutical Sciences, Anhui University of Chinese Medicine, Hefei, 230012, China.

*To whom correspondence should be addressed.

E-mail: haoehong@ahnu.edu.cn, iao421@ahnu.edu.cn

\section{Contents:}

1. Experimental details.................................................. 2

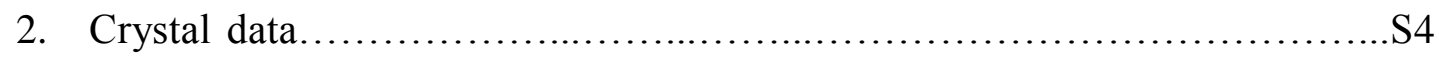

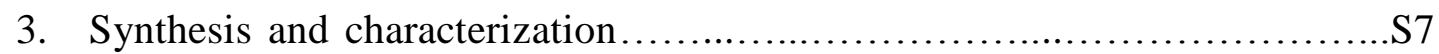

4. Photophysical data.................................................... 15

5. Electrochemical spectra............................................... 22

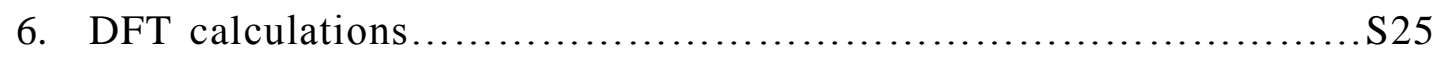

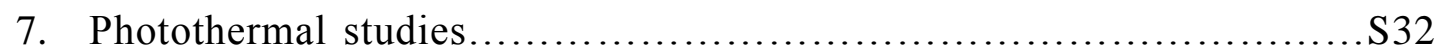

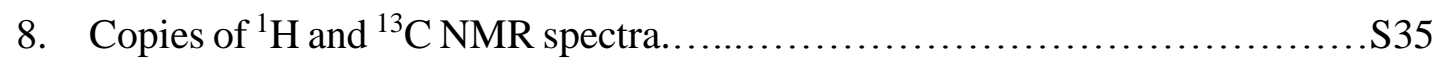

9. High resolution mass spectroscopes for all new compounds................S49 


\section{Experimental details}

General information. Reagents and solvents were used as received from commercial suppliers unless noted otherwise. All reactions were performed in oven-dried or flamedried glassware unless otherwise stated, and were monitored by TLC using $0.25 \mathrm{~mm}$ silica gel plates with UV indicator (60F-254). ${ }^{1} \mathrm{H}$ NMR and ${ }^{13} \mathrm{C}$ NMR spectra were recorded at $300 \mathrm{MHz}$ or $500 \mathrm{MHz}$ NMR spectrometer in $\mathrm{CDCl}_{3}$ at room temperature. Chemical shifts $(\delta)$ are given in ppm relative to $\mathrm{CDCl}_{3}\left(7.26 \mathrm{ppm}\right.$ for ${ }^{1} \mathrm{H}$ and $77 \mathrm{ppm}$ for $\left.{ }^{13} \mathrm{C}\right)$ to internal TMS ( $\left.\delta=0 \mathrm{ppm}\right)$ as internal standard. High-resolution mass spectra (HRMS) were obtained using ESI-TOF, APCI-TOF or MAIDI-TOF in positive mode.

Absorption and emission measurements. UV-visible absorption and fluorescence emission spectra were recorded on a commercial spectrophotometer (Shimadzu UV2450 and Edinburgh FS5 spectrometers, 190-900 nm scan range). Relative fluorescence quantum efficiencies of these BODIPYs were obtained by comparing the areas under the corrected emission spectrum of the test sample in various solvents with Cresyl violet perchlorate $(\Phi=0.54 \text { in methanol })^{1}$ or 1,7-diphenyl-3,5-di(4-methoxyphenyl)azadipyrromethene $(\Phi=0.36 \text { in chloroform })^{2}$ and Indocyanine Green $(\Phi=0.12$ in dimethyl sulfoxide). ${ }^{3}$ Non-degassed, spectroscopic grade solvents and a $10 \mathrm{~mm}$ quartz cuvette were used. Dilute solutions $(0.01<\mathrm{A}<0.05)$ were used to minimize the reabsorption effects. Quantum yields were determined using the following equation:

$$
\Phi_{x}=\Phi_{r} \times \frac{F_{x}}{F_{r}} \times \frac{1-10^{-A_{r}\left(\lambda_{\mathrm{ex}}\right)}}{1-10^{-A_{x}\left(\lambda_{\mathrm{ex}}\right)}} \times \frac{n_{x}^{2}}{n_{r}^{2}}
$$

Where the subscripts $x$ and $r$ refer respectively to sample $x$ and reference (standard) fluorophore $r$ with known quantum yield $\Phi_{r}$ in a specific solvent; $F$ stands for the spectrally corrected, integrated fluorescence spectra; $A\left(\lambda_{\text {ex }}\right)$ denotes the absorbance at the used excitation wavelength $\lambda_{\text {ex }} ; n$ represents the refractive index of the solvent (in principle at the average emission wavelength).

X-ray structure analysis. Crystal of FD4 suitable for X-ray analysis was obtained by slow evaporation of the dichloromethane solution. The vial containing this solution was 
placed, loosely capped, to promote the crystallization. A suitable crystal was chosen and mounted on a glass fiber using grease. The suitable crystals were selected and mounted on a suitable support. Data were collected using a Bruker APEX-II ${ }^{4}$ CCD diffractometer operating at $T=293(2) \mathrm{K}$. The determination of unit cell parameters and data collections were performed with Mo K $\alpha$ radiation $(\lambda)$ at $0.71073 \AA$. The total number of runs and images was based on the strategy calculation from the program APEX2 (Bruker). ${ }^{5}$ The structure was solved by the structure solution program Olex2 (Dolomanov et al., 2009) ${ }^{6}$ and the model was refined with version 2014/7 of ShelXL (Sheldrick, 2015) ${ }^{7}$ using full matrix least squares on $\mathbf{F}^{2}$ minimisation. CCDC-2100998 (DF4) contains the supplementary crystallographic data for this paper. These data can be obtained free of charge from The Cambridge Crystallographic Data Centre via www.ccdc.cam.ac.uk/data_request/cif.

Electrochemical measurements. Cyclic voltammograms of $1 \mathrm{mM}$ dyes M2, D4, FD2, T2 and T4 were measured in dichloromethane solution, containing $0.1 \mathrm{M} \mathrm{TBAPF}_{6}$ as the supporting electrolyte, glassy carbon electrode as a working electrode, Pt wire as a counter electrode, and saturated calomel electrode (SCE) as reference electrode at 100 $\mathrm{mV} \mathrm{s}^{-1}$ of scanning rate at room temperature. 


\section{Crystal data}

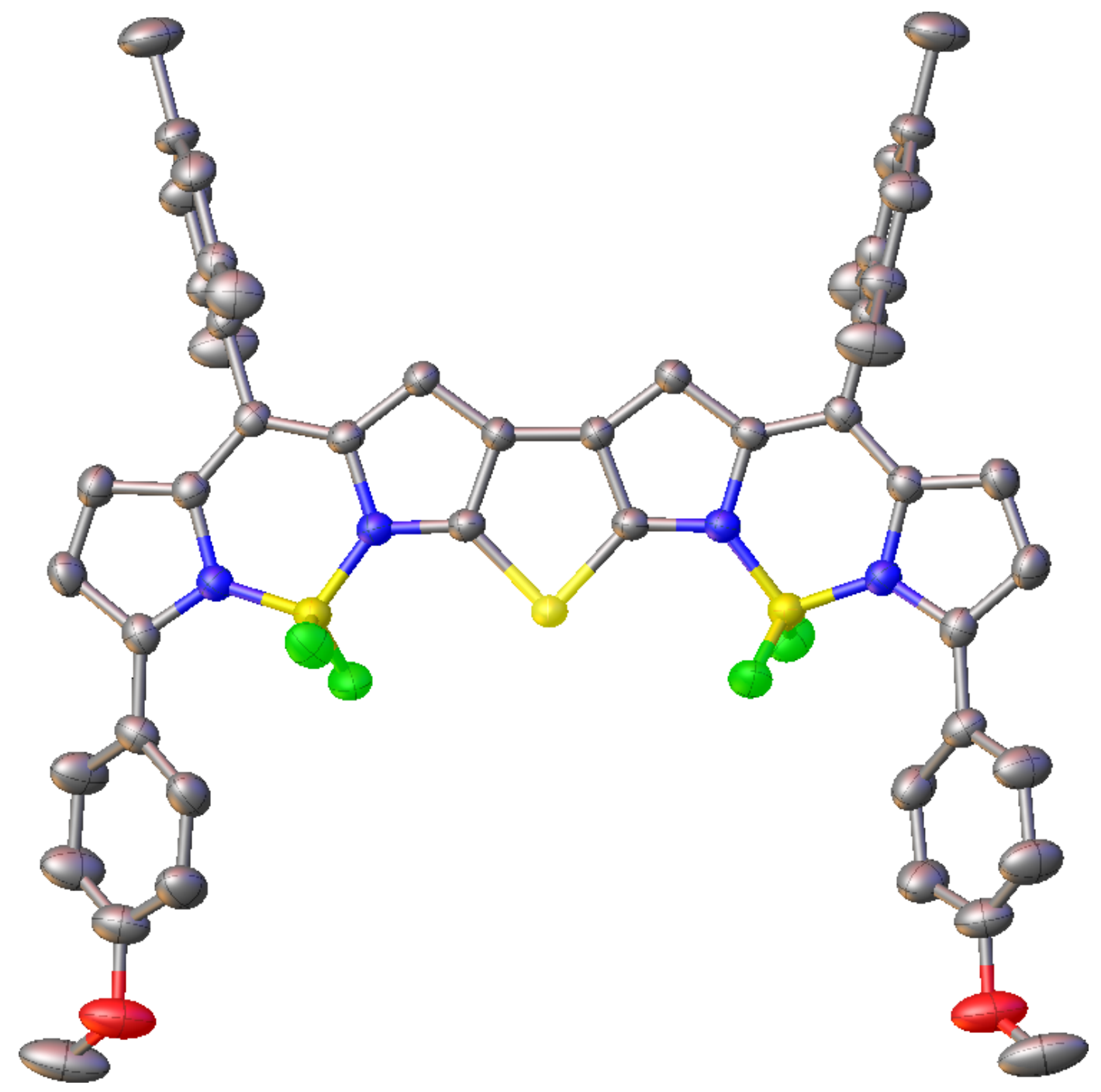

Figure S1. X-ray structures of FD4 with thermal ellipsoids shown at the 50\% level. Hydrogen atoms are omitted for clarity. 
Table S1. Selected geometrical parameters of compounds FD4 obtained from crystallography.

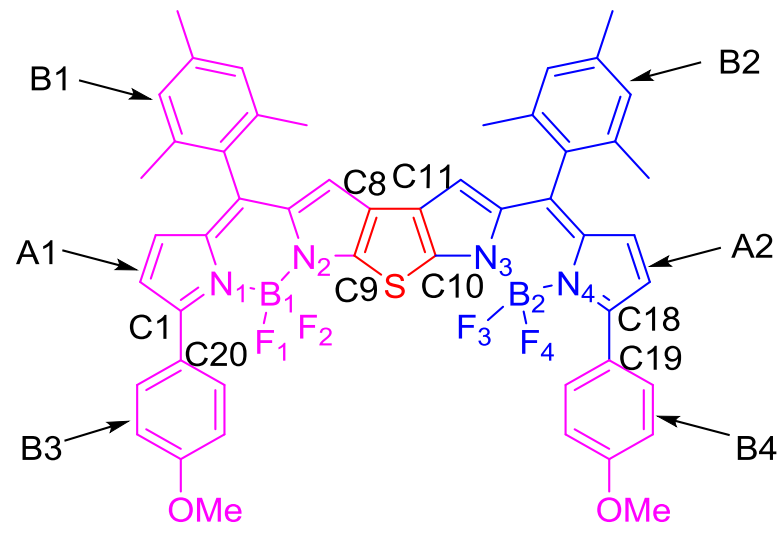

\begin{tabular}{|c|c|}
\hline & B2-3Cl \\
\hline \multirow[t]{4}{*}{ the $\mathrm{B}-\mathrm{N}$ bond distances $(\AA)$} & $1.555(4)$ \\
\hline & $1.549(4)$ \\
\hline & $1.549(4)$ \\
\hline & $1.555(4)$ \\
\hline \multirow[t]{4}{*}{ the $\mathrm{B}-\mathrm{F}$ bond distances $(\AA)$} & $1.394(4)$ \\
\hline & $1.380(4)$ \\
\hline & $1.380(4)$ \\
\hline & $1.394(4)$ \\
\hline the C9-S bond distances $(\AA)$ & $1.743(3)$ \\
\hline the C10-S bond distances $(\AA)$ & $1.743(3)$ \\
\hline the $\mathrm{C} 8-\mathrm{C} 11$ bond distances $(\AA)$ & $1.437(6)$ \\
\hline the $\mathrm{C} 1-\mathrm{C} 20$ bond distances $(\AA)$ & $1.462(4)$ \\
\hline the $\mathrm{C} 18-\mathrm{C} 19$ bond distances $(\AA)$ & $1.462(4)$ \\
\hline $\begin{array}{l}\text { dihedral angles between meso-aryl B1 and dipyrrin } \\
\text { core A1 (deg) }\end{array}$ & $75.84(10)$ \\
\hline $\begin{array}{l}\text { dihedral angles between meso-aryl B2 and dipyrrin } \\
\text { core A2 (deg) }\end{array}$ & $75.84(10)$ \\
\hline $\begin{array}{l}\text { dihedral angles between meso-aryl B3 and dipyrrin } \\
\text { core A1 (deg) }\end{array}$ & $35.56(11)$ \\
\hline $\begin{array}{l}\text { dihedral angles between meso-aryl B4 and dipyrrin } \\
\text { core A2 (deg) }\end{array}$ & $35.56(11)$ \\
\hline $\begin{array}{l}\text { dihedral angles between dipyrrin core A1 and } \\
\text { dipyrrin core A2 (deg) }\end{array}$ & $0.08(10)$ \\
\hline
\end{tabular}


Table S2. Crystal data and structure refinement for FD4.

Empirical formula

Formula weight

Temperature/K

Crystal system

Space group

$\mathrm{a} / \AA$

$\mathrm{b} / \AA$

$\mathrm{c} / \AA$

$\alpha /{ }^{\circ}$

$\beta /{ }^{\circ}$

$\gamma /{ }^{\circ}$

Volume $/ \AA^{3}$

Z

$\rho_{\text {calc }} \mathrm{g} / \mathrm{cm}^{3}$

$\mu / \mathrm{mm}^{-1}$

$\mathrm{F}(000)$

Crystal size $/ \mathrm{mm}^{3}$

Radiation

$2 \Theta$ range for data collection $/{ }^{\circ}$

Index ranges

Reflections collected

Independent reflections

Data/restraints/parameters

Goodness-of-fit on $\mathrm{F}^{2}$

Final $\mathrm{R}$ indexes $[\mathrm{I}>=2 \sigma(\mathrm{I})]$

Final R indexes [all data]

Largest diff. peak/hole / e $\AA^{-3}$
$\mathrm{C}_{50} \mathrm{H}_{42} \mathrm{~B}_{2} \mathrm{~F}_{4} \mathrm{~N}_{4} \mathrm{O}_{2} \mathrm{~S}$

860.55

273.15

monoclinic

$\mathrm{C} 2 / \mathrm{c}$

$13.729(2)$

22.899(2)

14.2211(14)

90

102.799(7)

90

4359.5(9)

4

1.311

0.137

1792.0

$0.3 \times 0.3 \times 0.06$

$\operatorname{MoK} \alpha(\lambda=0.71073)$

6.086 to 55.282

$-17 \leq \mathrm{h} \leq 17,-29 \leq \mathrm{k} \leq 29,-18 \leq 1 \leq 18$

63326

$5017\left[\mathrm{R}_{\text {int }}=0.0938, \mathrm{R}_{\text {sigma }}=0.0399\right]$

$5017 / 0 / 289$

1.096

$\mathrm{R}_{1}=0.0842, \mathrm{wR}_{2}=0.2137$

$\mathrm{R}_{1}=0.1174, \mathrm{wR}_{2}=0.2487$

$0.78 /-0.34$ 


\section{Syntheis and characterization}

BODIPYs M1 and M2 was synthesized according to the literature. ${ }^{8}$

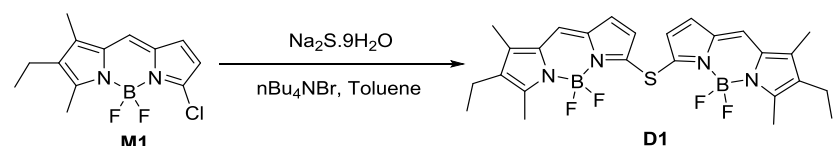

Synthesis of BODIPY D1: To a solution of M1 (56 mg, $0.2 \mathrm{mmol})$ in toluene (2 mL) was added water $(0.05 \mathrm{~mL}), \mathrm{nBu} 4 \mathrm{NBr}(12 \mathrm{mg}, 0.04 \mathrm{mmol})$ and $\mathrm{Na}_{2} \mathrm{~S} \cdot 9 \mathrm{H}_{2} \mathrm{O}(26 \mathrm{mg}$, $0.11 \mathrm{mmol}$ ). The reaction mixture was stirred at $80{ }^{\circ} \mathrm{C}$ in oil bath under $\operatorname{Ar}$ for $3 \mathrm{~h}$, then the reaction mixture was poured into water and was extracted with dichloromethane (30 $\mathrm{mL} \times 3$ ). Organic layers were combined, dried over anhydrous $\mathrm{Na}_{2} \mathrm{SO}_{4}$, and organic solvent was removed under vacuum. The crude product was purified by column chromatography on silica gel (eluent: hexane/dichloromethane $=1: 1$ ) to afford the desired BODIPY D1 (19 mg, 36\%) as dark violet solid. ${ }^{1} \mathrm{H}$ NMR $\left(500 \mathrm{MHz}, \mathrm{CDCl}_{3}\right) \delta$ : $6.97(\mathrm{~s}, 2 \mathrm{H}), 6.77(\mathrm{~d}, J=3.7 \mathrm{~Hz}, 2 \mathrm{H}), 6.34(\mathrm{~d}, J=3.8 \mathrm{~Hz}, 2 \mathrm{H}), 2.57$ (s, 6H), 2.39 (q, $J$ $=7.4 \mathrm{~Hz}, 4 \mathrm{H}), 2.16(\mathrm{~s}, 6 \mathrm{H}), 1.06(\mathrm{t}, J=7.5 \mathrm{~Hz}, 6 \mathrm{H}),{ }^{13} \mathrm{C} \mathrm{NMR}\left(126 \mathrm{MHz}, \mathrm{CDCl}_{3}\right) \delta$ : $162.6,144.2,139.9,136.0,134.7,134.3,125.5,121.4,120.8,17.2,14.2,13.2,9.4$. HRMS(APCI) calcd. for $\mathrm{C}_{26} \mathrm{H}_{28} \mathrm{~B}_{2} \mathrm{~F}_{4} \mathrm{~N}_{4} \mathrm{~S}[\mathrm{M}+\mathrm{H}]^{+}:$527.2235, found 527.2228.

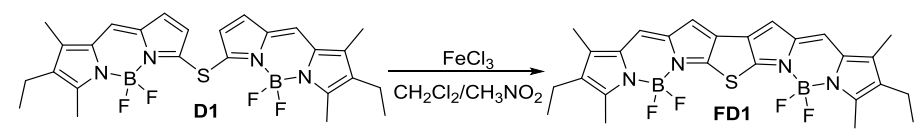

Synthesis of BODIPY FD1: To a solution of D1 (53 mg, $0.1 \mathrm{mmol})$ in $10 \mathrm{~mL}$ of dry dichloromethane was dropwisely added $\mathrm{FeCl}_{3}(162 \mathrm{mg}, 1.0 \mathrm{mmol})$ in $1 \mathrm{~mL}$ of $\mathrm{CH}_{3} \mathrm{NO}_{2}$. The reaction mixture was stirred at room temperature, was monitored by TLC, and was quenched by adding saturated aqueous solution of $\mathrm{NaHCO}_{3}$. The reaction mixture was diluted with dichloromethane, washed twice with water, dried over anhydrous $\mathrm{Na}_{2} \mathrm{SO}_{4}$, and evaporated under vacuum. The crude product was purified by column chromatography on silica gel (eluent: hexane/dichloromethane $=1: 1$ ) to afford the desired BODIPY FD1 (27 mg, 52\%) as dark violet solid. ${ }^{1} \mathrm{H}$ NMR (300 MHz, $\mathrm{CDCl}_{3}$ ) $\delta: 7.03(\mathrm{~s}, 2 \mathrm{H}), 6.86(\mathrm{~s}, 2 \mathrm{H}), 2.58(\mathrm{~s}, 6 \mathrm{H}), 2.41(\mathrm{q}, J=6.5 \mathrm{~Hz}, 4 \mathrm{H}), 2.18(\mathrm{~s}, 6 \mathrm{H}), 1.09$ (t, 
$J=6.3 \mathrm{~Hz}, 6 \mathrm{H}) .{ }^{13} \mathrm{C}$ NMR was not available due to its poor solubility. HRMS (MALDITOF) calcd. for $\mathrm{C}_{26} \mathrm{H}_{26} \mathrm{~B}_{2} \mathrm{~F}_{4} \mathrm{~N}_{4} \mathrm{~S}[\mathrm{M}]^{+}$: 524.2004, found 524.2009.

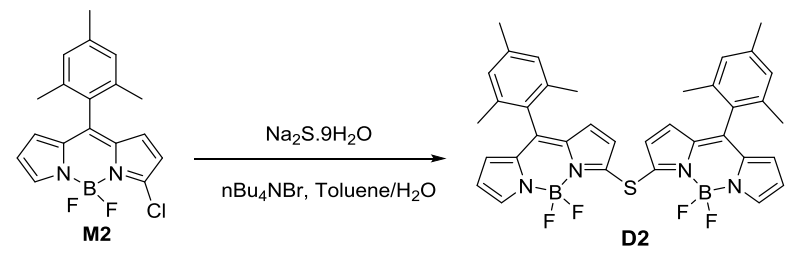

Synthesis of BODIPY D2: To a solution of M2 $(69 \mathrm{mg}, 0.2 \mathrm{mmol})$ in toluene (2 mL) was added water $(0.05 \mathrm{~mL}), \mathrm{nBu} 4 \mathrm{NBr}(12 \mathrm{mg}, 0.04 \mathrm{mmol})$ and $\mathrm{Na}_{2} \mathrm{~S} \cdot 9 \mathrm{H}_{2} \mathrm{O}(26 \mathrm{mg}$, $0.11 \mathrm{mmol}$ ) using the same procedure described for D1. The crude product was purified by column chromatography on silica gel (eluent: hexane/dichloromethane $=1: 1$ ) to afford the desired BODIPY D2 (55 mg, 84\%) as dark violet solid. ${ }^{1} \mathrm{H}$ NMR (500 MHz, $\left.\mathrm{CDCl}_{3}\right) \delta: 7.91(\mathrm{~s}, 2 \mathrm{H}), 6.95(\mathrm{~s}, 4 \mathrm{H}), 6.64(\mathrm{~d}, J=4.1 \mathrm{~Hz}, 2 \mathrm{H}), 6.61(\mathrm{~d}, J=4.3 \mathrm{~Hz}, 2 \mathrm{H})$, 6.51-6.44 (m, 4H), 2.35 (s, 6H), $2.11(\mathrm{~s}, 12 \mathrm{H}) .{ }^{13} \mathrm{C} \mathrm{NMR}\left(126 \mathrm{MHz}, \mathrm{CDCl}_{3}\right) \delta: 149.9$, 144.7, 144.3, 138.9, 136.9, 136.5, 135.5, 129.5, 129.4, 129.4, 128.2, 122.7, 118.8, 21.1, 20.0. HRMS (ESI) calcd. for $\mathrm{C}_{36} \mathrm{H}_{32} \mathrm{~B}_{2} \mathrm{~F}_{4} \mathrm{~N}_{4} \mathrm{~S}[\mathrm{M}-\mathrm{F}]^{+}$: 631.2480, found 631.2484.

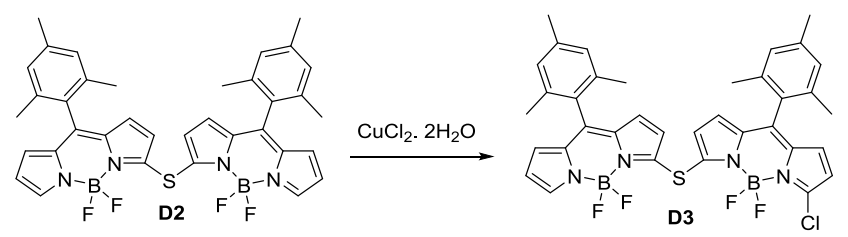

Syntheses of BODIPY D3: A mixture of BODIPY D2 (65 mg, $0.1 \mathrm{mmol}$ ) and $\mathrm{CuCl}_{2} .2 \mathrm{H}_{2} \mathrm{O}(102 \mathrm{mg}, 0.6 \mathrm{mmol})$ in $\mathrm{CH}_{3} \mathrm{CN}(20 \mathrm{~mL})$ was stirred under air at $80^{\circ} \mathrm{C}$ in oil bath for $2 \mathrm{~h}$. After cooling down to room temperature, the reaction mixture was poured in dichloromethane $(30 \mathrm{~mL})$, washed with water $(3 \times 50 \mathrm{~mL})$, dried over anhydrous $\mathrm{Na}_{2} \mathrm{SO}_{4}$, filtered and evaporated under vacuum. The crude product was purified through column chromatography on silica gel (eluent: hexane/dichloromethane $=1: 1)$ to afford the desired BODIPY D3 (34 mg, 53\%) as dark violet solid. ${ }^{1} \mathrm{H}$ NMR $\left(500 \mathrm{MHz}, \mathrm{CDCl}_{3}\right) \delta: 7.92(\mathrm{~s}, 1 \mathrm{H}), 6.94(\mathrm{~d}, J=3.7 \mathrm{~Hz}, 4 \mathrm{H}), 6.65(\mathrm{~d}, J=4.1 \mathrm{~Hz}, 1 \mathrm{H})$, $6.61(\mathrm{~d}, J=4.3 \mathrm{~Hz}, 1 \mathrm{H}), 6.57(\mathrm{dd}, J=7.9,4.4 \mathrm{~Hz}, 2 \mathrm{H}), 6.49(\mathrm{dd}, J=5.8,3.1 \mathrm{~Hz}, 2 \mathrm{H})$, $6.46(\mathrm{~d}, J=4.4 \mathrm{~Hz}, 1 \mathrm{H}), 6.35(\mathrm{~d}, J=4.3 \mathrm{~Hz}, 1 \mathrm{H}), 2.35$ (s, 3H), 2.34 (s, 3H), 2.11 (s, 
6H), $2.10(\mathrm{~d}, 6 \mathrm{H}) .{ }^{13} \mathrm{C} \mathrm{NMR}\left(126 \mathrm{MHz}, \mathrm{CDCl}_{3}\right) \delta: 151.7,149.4,145.4,145.1,144.4$, 143.0, 139.5, 139.4, 137.3, 137.1, 136.9, 136.0, 134.7, 130.1, 129.8, 129.7, 129.1, 128.7, 128.6, 123.5, 123.3, 119.4, 119.0, 21.5, 20.4, 20.4. HRMS (ESI) calcd. for $\mathrm{C}_{36} \mathrm{H}_{31} \mathrm{~B}_{2} \mathrm{ClF}_{3} \mathrm{~N}_{4} \mathrm{~S}[\mathrm{M}-\mathrm{F}]^{+}:$665.2096, found 665.2097.

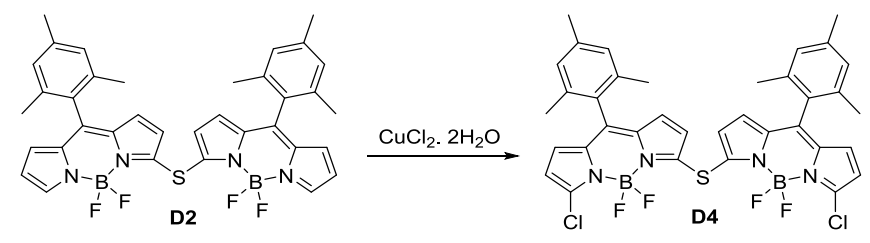

BODIPY D4 was obtained from BODIPY D2 using the same procedure described for D3, but the $\mathrm{CuCl}_{2} .2 \mathrm{H}_{2} \mathrm{O}$ (136 mg, $\left.0.8 \mathrm{mmol}\right)$ was used, and this reaction was completed after $5 \mathrm{~h}$, the crude product was purified through column chromatography on silica gel (eluent: hexane/dichloromethane $=1: 1)$ to afford a dark violet solid D4 $(33 \mathrm{mg}, 46 \%)$. ${ }^{1} \mathrm{H}$ NMR (500 MHz, CDCl 3 ) $\delta: 6.94(\mathrm{~s}, 4 \mathrm{H}), 6.58(\mathrm{~d}, J=4.1 \mathrm{~Hz}, 4 \mathrm{H}), 6.48$ (d, $J=4.3$ $\mathrm{Hz}, 2 \mathrm{H}), 6.36(\mathrm{~d}, J=4.3 \mathrm{~Hz}, 2 \mathrm{H}), 2.35$ (s, 6H), 2.10 (s, 12H). ${ }^{13} \mathrm{C}$ NMR $(126 \mathrm{MHz}$, $\left.\mathrm{CDCl}_{3}\right) \delta: 150.2,144.5,142.9,139.1,136.6,134.4,129.6,129.3,128.6,128.3,123.3$, 118.8, 21.1, 20.0. HRMS (ESI) calcd. for $\mathrm{C}_{36} \mathrm{H}_{30} \mathrm{~B}_{2} \mathrm{Cl}_{2} \mathrm{~F}_{3} \mathrm{~N}_{4} \mathrm{~S}$ [M-F] $]^{+}$: 699.1701, found 699.1707.

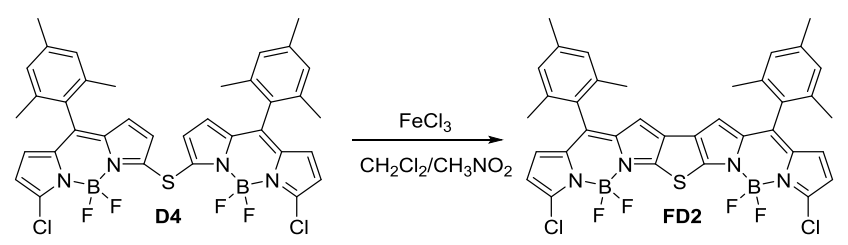

Synthesis of BODIPY FD2: BODIPY FD2 as a dark violet solid was prepared in 78\% yield (56 mg) from BODIPY D4 (72 mg, $0.1 \mathrm{mmol})$ and $\mathrm{FeCl}_{3}(162 \mathrm{mg}, 1 \mathrm{mmol})$ using the same procedure described for FD2. The crude product was purified through column chromatography on silica gel (eluent: hexane/dichloromethane $=1: 1) .{ }^{1} \mathrm{H}$ NMR (500 $\left.\mathrm{MHz}, \mathrm{CDCl}_{3}\right) \delta: 6.94(\mathrm{~s}, 4 \mathrm{H}), 6.56(\mathrm{~d}, J=4.2 \mathrm{~Hz}, 2 \mathrm{H}), 6.48(\mathrm{~s}, 2 \mathrm{H}), 6.34(\mathrm{~d}, J=4.2 \mathrm{~Hz}$, 2H), 2.34 (s, 6H), 2.10 (s, 12H). $\left.{ }^{13} \mathrm{C} \mathrm{NMR} \mathrm{(126} \mathrm{MHz,} \mathrm{CDCl}_{3}\right) \delta: 162.4,143.4,143.3$, 139.7, 139.2, 136.7, 134.5, 129.5, 128.4, 128.3, 126.7, 118.3, 118.0, 21.1, 19.9. HRMS (ESI) calcd. for $\mathrm{C}_{36} \mathrm{H}_{28} \mathrm{~B}_{2} \mathrm{Cl}_{2} \mathrm{~F}_{4} \mathrm{~N}_{4} \mathrm{SNa}[\mathrm{M}+\mathrm{Na}]^{+}$: 739.1426, found739.1427. 

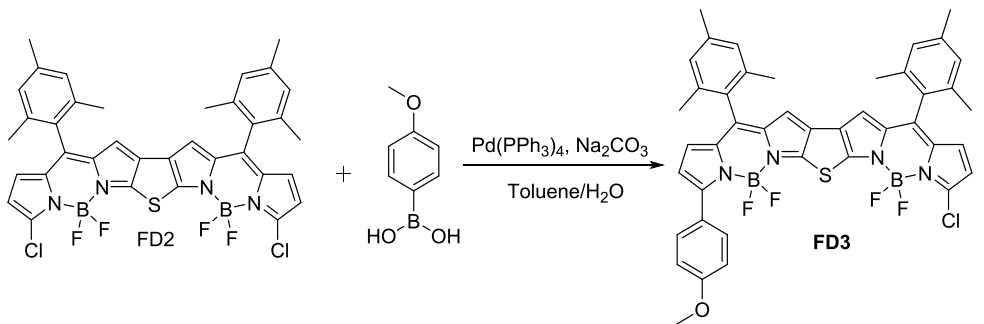

Synthesis of BODIPY FD3: To a dry round-bottomed flask loaded with compound FD2 (0.1 mmol, $72 \mathrm{mg}$ ), (4-methoxyphenyl) boronic acid (0.1 mmol, $15 \mathrm{mg}), \mathrm{Na}_{2} \mathrm{CO}_{3}(3 \mathrm{ml}$, $1 \mathrm{M})$, and $\mathrm{Pd}\left(\mathrm{PPh}_{3}\right)_{4}(0.01 \mathrm{mmol}, 11 \mathrm{mg})$ was added toluene $(5 \mathrm{~mL})$. The freeze-pumpthaw cycle was carried out three times. After that, the mixture was heated to $80{ }^{\circ} \mathrm{C}$ in oil bath under argon and stirred for $10 \mathrm{~h}$. After cooling to room temperature, the reaction mixture was extracted with $\mathrm{CH}_{2} \mathrm{Cl}_{2}$ and dried over anhydrous $\mathrm{Na}_{2} \mathrm{SO}_{4}$. The organic layers were combined and evaporated under vacuum. The crude product was purified through column chromatography ( to afford FD3 as a blackish solid in $20 \%$ yield $(16 \mathrm{mg}) .{ }^{1} \mathrm{H}$ NMR $\left(500 \mathrm{MHz}, \mathrm{CDCl}_{3}\right) \delta$ : $8.00(\mathrm{~d}, J=8.8 \mathrm{~Hz}, 2 \mathrm{H}), 7.04(\mathrm{~d}, J=8.9 \mathrm{~Hz}, 2 \mathrm{H}), 6.94(\mathrm{~d}, J=9.7 \mathrm{~Hz}, 4 \mathrm{H}), 6.66(\mathrm{dd}, J$ $=10.5,4.4 \mathrm{~Hz}, 2 \mathrm{H}), 6.49(\mathrm{~d}, J=4.1 \mathrm{~Hz}, 1 \mathrm{H}), 6.42(\mathrm{~d}, J=13.5 \mathrm{~Hz}, 2 \mathrm{H}), 6.30(\mathrm{~d}, J=4.2$ $\mathrm{Hz}, 1 \mathrm{H}), 3.91(\mathrm{~s}, 3 \mathrm{H}), 2.35(\mathrm{~d}, J=7.0 \mathrm{~Hz}, 6 \mathrm{H}), 2.15(\mathrm{~s}, 6 \mathrm{H}), 2.10(\mathrm{~s}, 6 \mathrm{H}) .{ }^{13} \mathrm{C} \mathrm{NMR}$ $\left(126 \mathrm{MHz}, \mathrm{CDCl}_{3}\right) \delta: 164.0,161.6,160.6,157.7,142.5,142.0,141.8,139.8,139.0$, 138.8, 138.6, 138.1, 136.8, 136.7, 134.2, 131.4, 131.2, 129.5, 128.6, 128.4, 128.2, 128.2, $124.7,124.0,121.3,117.5,117.3,115.9,114.1,55.4,21.1,20.0,19.9$. HRMS (APCI) calcd. for $\mathrm{C}_{43} \mathrm{H}_{35} \mathrm{~B}_{2} \mathrm{ClF}_{3} \mathrm{~N}_{4} \mathrm{OS}[\mathrm{M}-\mathrm{F}]^{+}$: 769.2358, found 769.2367.
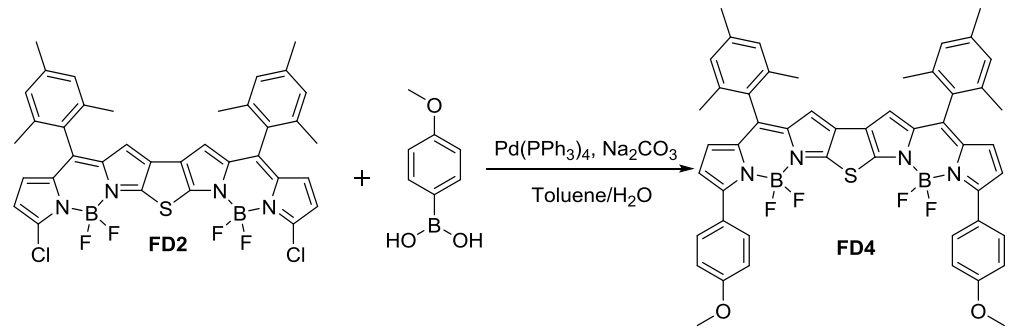

Synthesis of BODIPY FD4: BODIPY FD4 as a blackish solid was prepared in $18 \%$ yield (16 mg) from compound FD2 (72 mg, $0.1 \mathrm{mmol})$, (4-methoxyphenyl) boronic acid $(0.2 \mathrm{mmol}, 30 \mathrm{mg}), \mathrm{Na}_{2} \mathrm{CO}_{3}(5 \mathrm{mml}, 1 \mathrm{M})$, and $\mathrm{Pd}\left(\mathrm{PPh}_{3}\right)_{4}(0.01 \mathrm{mmol}, 11 \mathrm{mg})$ 
using the same procedure described for FD3. The crude product was purified through column chromatography on silica gel (eluent: petroleum ether/dichloromethane $=2: 1$ ) . ${ }^{1} \mathrm{H} \mathrm{NMR}\left(500 \mathrm{MHz}, \mathrm{CDCl}_{3}\right) \delta: 8.01(\mathrm{~d}, J=8.9 \mathrm{~Hz}, 4 \mathrm{H}), 7.00(\mathrm{~d}, J=8.9 \mathrm{~Hz}, 4 \mathrm{H}), 6.95$ (s, 4H), $6.63(\mathrm{~s}, 4 \mathrm{H}), 6.46(\mathrm{~d}, J=4.2 \mathrm{~Hz}, 2 \mathrm{H}), 6.40(\mathrm{~d}, J=4.2 \mathrm{~Hz}, 2 \mathrm{H}), 3.87(\mathrm{~s}, 6 \mathrm{H})$, 2.35 (s, 6H), 2.14 (s, 12H). ${ }^{13} \mathrm{C}$ NMR (126 MHz, $\left.\mathrm{CDCl}_{3}\right) \delta: 161.3,159.4,159.3,141.7$, 138.6, 137.7, 136.9, 131.2, 130.3, 129.7, 128.1, 126.1, 124.4, 120.5, 115.5, 114.0, 55.4, 21.1, 20.0. HRMS (ESI) calcd. for $\mathrm{C}_{50} \mathrm{H}_{42} \mathrm{~B}_{2} \mathrm{~F}_{3} \mathrm{~N}_{4} \mathrm{O}_{2} \mathrm{~S}$ [M-F] $]^{+}$: 841.3167, found 841.3152 .
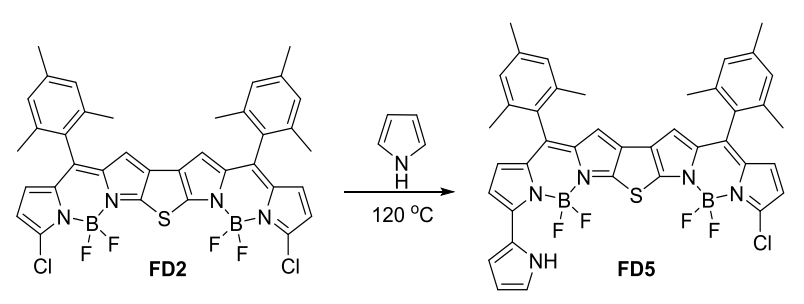

Synthesis of BODIPY FD5: BODIPY FD2 (72 mg, $0.1 \mathrm{mmol})$ was dissolved in pyrrole $(5 \mathrm{~mL})$ and stirred under argon at $120{ }^{\circ} \mathrm{C}$ in oil bath for $2 \mathrm{~h}$. After cooling down to room temperature, the reaction mixture was extracted with $\mathrm{CH}_{2} \mathrm{Cl}_{2}(30 \times 3 \mathrm{~mL})$. The organic layer was dried over anhydrous $\mathrm{Na}_{2} \mathrm{SO}_{4}$, filtered, and evaporated to dryness under vacuum. The crude product was purified through column chromatography on silica using petroleum ether/dichloromethane $(1: 1, \mathrm{v} / \mathrm{v})$ as eluent, from which the desired product FD5 as a blackish solid was obtained in 62\% yield (48 mg). ${ }^{1} \mathrm{H}$ NMR (500 $\left.\mathrm{MHz} \mathrm{CDCl}_{3}\right) \delta: 10.50(\mathrm{~s}, 1 \mathrm{H}), 7.28(\mathrm{~s}, 1 \mathrm{H}), 7.04(\mathrm{~s}, 1 \mathrm{H}), 6.94(\mathrm{~d}, J=3.5 \mathrm{~Hz}, 4 \mathrm{H}), 6.87$ $(\mathrm{d}, J=4.7 \mathrm{~Hz}, 1 \mathrm{H}), 6.64(\mathrm{~d}, J=4.7 \mathrm{~Hz}, 1 \mathrm{H}), 6.46(\mathrm{~d}, J=4.0 \mathrm{~Hz}, 1 \mathrm{H}), 6.42(\mathrm{~s}, 2 \mathrm{H}), 6.30$ $(\mathrm{s}, 1 \mathrm{H}), 6.28(\mathrm{~d}, J=4.1 \mathrm{~Hz}, 1 \mathrm{H}), 2.34(\mathrm{~s}, 6 \mathrm{H}), 2.12(\mathrm{~s}, 6 \mathrm{H}), 2.11(\mathrm{~s}, 6 \mathrm{H}) .{ }^{13} \mathrm{C} \mathrm{NMR}(126$ $\left.\mathrm{MHz}, \mathrm{CDCl}_{3}\right) \delta: 165.0,153.0,152.0,142.5,141.4,140.2,139.3,139.0,138.9,138.4$, 137.6, 137.4, 137.2, 134.4, 132.0, 130.0, 129.5, 129.2 128.6, 128.3, 127.7, 123.9, 123.4, $122.0,119.7,117.5,117.2,113.8,112.5,21.5,20.4$. HRMS (APCI) calcd. for $\mathrm{C}_{40} \mathrm{H}_{32} \mathrm{~B}_{2} \mathrm{ClF}_{4} \mathrm{~N}_{5} \mathrm{~S}[\mathrm{M}+\mathrm{H}]^{+}:$748.2267, found 748.2258. 

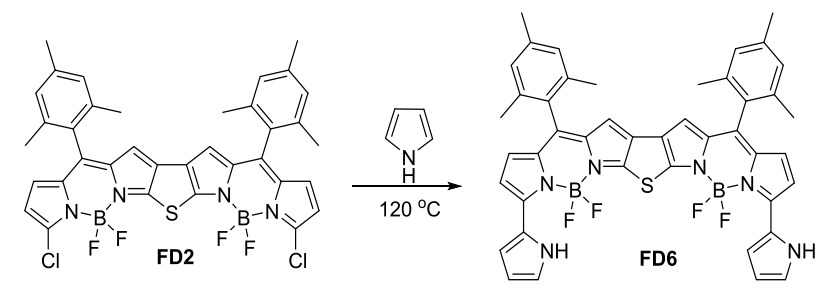

Synthesis of BODIPY FD6: BODIPY FD6 as a blackish solid was prepared using the above procedure from compound FD2 $(72 \mathrm{mg}, 0.1 \mathrm{mmol})$, pyrrole $(5 \mathrm{~mL})$ and stirred under argon at $120{ }^{\circ} \mathrm{C}$ in oil bath for $4 \mathrm{~h}$ affording FD6 in $56 \%$ yield $(43 \mathrm{mg}$ ). The crude product was purified through column chromatography on silica gel (eluent: petroleum ether/dichloromethane $=2: 1) .{ }^{1} \mathrm{H}$ NMR $\left(500 \mathrm{MHz}, \mathrm{CDCl}_{3}\right) \delta: 10.48(\mathrm{~s}, 2 \mathrm{H}), 7.23(\mathrm{dd}$, $J=2.7,1.4 \mathrm{~Hz}, 2 \mathrm{H}), 6.99(\mathrm{dd}, J=3.8,2.4 \mathrm{~Hz}, 2 \mathrm{H}), 6.94(\mathrm{~s}, 4 \mathrm{H}), 6.82(\mathrm{~d}, J=4.6 \mathrm{~Hz}$ 2H), $6.60(\mathrm{~d}, J=4.6 \mathrm{~Hz}, 2 \mathrm{H}), 6.40(\mathrm{dd}, J=6.1,2.3 \mathrm{~Hz}, 2 \mathrm{H}), 6.31(\mathrm{~s}, 2 \mathrm{H}), 2.35(\mathrm{~s}, 6 \mathrm{H})$, 2.13 (s, $12 \mathrm{H}) .{ }^{13} \mathrm{C} \mathrm{NMR}\left(126 \mathrm{MHz}, \mathrm{CDCl}_{3}\right) \delta: 154.7,150.8,138.9,138.3,138.2,137.9$, $137.4,131.2,130.3,128.5,126.5,125.7,124.2,120.9,118.3,113.5,112.0,21.5,20.4$. HRMS(APCI) calcd. for $\mathrm{C}_{44} \mathrm{H}_{36} \mathrm{~B}_{2} \mathrm{~F}_{4} \mathrm{~N}_{6} \mathrm{~S}[\mathrm{M}+\mathrm{H}]^{+}$: 779.2923, found 779.2906.

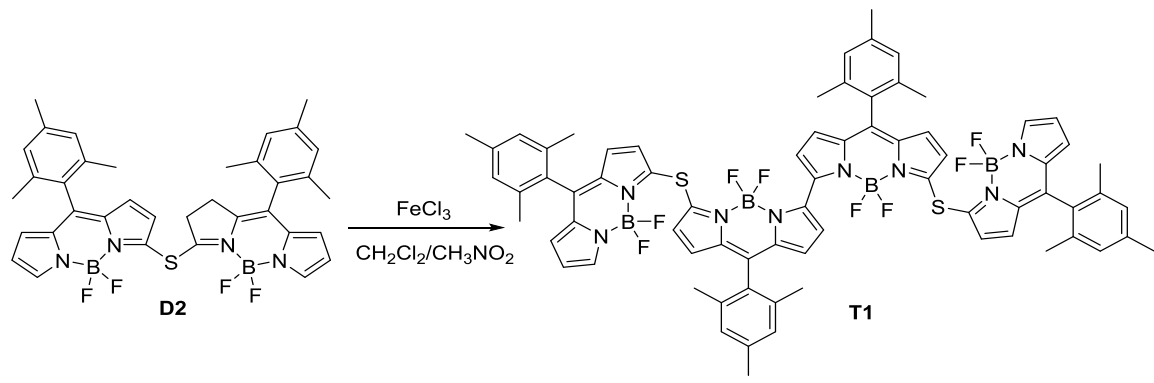

Synthesis of BODIPY T1: BODIPY T1 as a blackish solid was prepared in $80 \%$ yield (52 mg) from BODIPY D2 (65 mg, $0.1 \mathrm{mmol})$ and $\mathrm{FeCl}_{3}(162 \mathrm{mg}, 1 \mathrm{mmol})$ using the same procedure described for FD1. The crude product was purified through column chromatography on silica gel (eluent: petroleum ether/dichloromethane $=1: 1$ ). ${ }^{1} \mathrm{H}$ NMR $\left(500 \mathrm{MHz}, \mathrm{CDCl}_{3}\right) \delta: 7.89(\mathrm{~s}, 2 \mathrm{H}), 7.68(\mathrm{~d}, J=4.6 \mathrm{~Hz}, 2 \mathrm{H}), 6.95(\mathrm{~d}, J=11.8 \mathrm{~Hz}, 8 \mathrm{H})$, $6.67(\mathrm{~d}, J=4.6 \mathrm{~Hz}, 2 \mathrm{H}), 6.62(\mathrm{~d}, J=4.0 \mathrm{~Hz}, 2 \mathrm{H}), 6.59(\mathrm{dd}, J=4.3,1.6 \mathrm{~Hz}, 4 \mathrm{H}), 6.47$ $(\mathrm{dd}, J=4.2,2.1 \mathrm{~Hz}, 6 \mathrm{H}), 2.36(\mathrm{~s}, 12 \mathrm{H}), 2.35(\mathrm{~s}, 12 \mathrm{H}), 2.16(\mathrm{~s}, 12 \mathrm{H}), 2.09(\mathrm{~s}, 12 \mathrm{H}) .{ }^{13} \mathrm{C}$ NMR (126 MHz, $\left.\mathrm{CDCl}_{3}\right) \delta:$ 150.2, 148.0, 144.6, 144.2, 142.3, 138.9, 138.7, 137.4, $137.0,136.9,136.5,135.5,129.6,129.5,129.4,129.3,129.2,128.7,128.3,128.2,125.3$, 
123.5, 123.0, 118.7, 21.2, 21.1, 20.3, 20.0. HRMS (MALDI-TOF) calcd. for $\mathrm{C}_{72} \mathrm{H}_{62} \mathrm{~B}_{4} \mathrm{~F}_{8} \mathrm{~N}_{8} \mathrm{~S}_{2}[\mathrm{M}]^{+}:$1298.4783, found 1298.4784 .
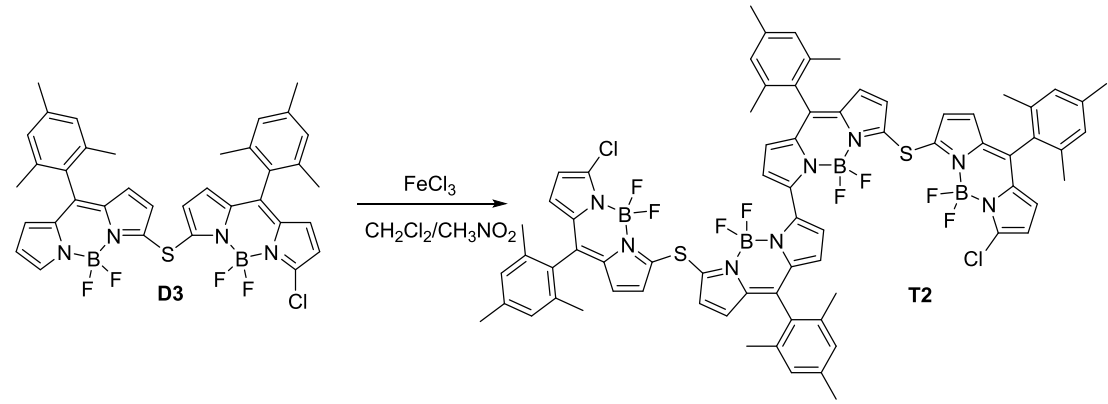

Synthesis of BODIPY T2: BODIPY T2 as a blackish solid was prepared in $65 \%$ yield (45 mg) from BODIPY D3 (68 mg, $0.1 \mathrm{mmol})$ and $\mathrm{FeCl}_{3}(162 \mathrm{mg}, 1 \mathrm{mmol})$ using the same procedure described for FD1. The crude product was purified through column chromatography on silica gel (eluent: petroleum ether/dichloromethane $=1: 1$ ). ${ }^{1} \mathrm{H}$ NMR $\left(500 \mathrm{MHz}, \mathrm{CDCl}_{3}\right) \delta: 7.68(\mathrm{~d}, J=4.6 \mathrm{~Hz}, 2 \mathrm{H}), 6.97(\mathrm{~s}, 4 \mathrm{H}), 6.93(\mathrm{~s}, 4 \mathrm{H}), 6.68(\mathrm{~d}, J=$ $4.6 \mathrm{~Hz}, 2 \mathrm{H}), 6.60(\mathrm{~d}, J=4.3 \mathrm{~Hz}, 2 \mathrm{H}), 6.57(\mathrm{~d}, J=4.4 \mathrm{~Hz}, 2 \mathrm{H}), 6.55(\mathrm{~d}, J=4.3 \mathrm{~Hz}, 2 \mathrm{H})$, $6.50(\mathrm{~d}, J=4.3 \mathrm{~Hz}, 2 \mathrm{H}), 6.48(\mathrm{~d}, J=4.4 \mathrm{~Hz}, 2 \mathrm{H}), 6.34(\mathrm{~d}, J=4.3 \mathrm{~Hz}, 2 \mathrm{H}), 2.37(\mathrm{~s}, 6 \mathrm{H})$, $2.34(\mathrm{~s}, 6 \mathrm{H}), 2.16(\mathrm{~s}, 12 \mathrm{H}), 2.09$ (s, 12H). ${ }^{13} \mathrm{C} \mathrm{NMR}\left(126 \mathrm{MHz}, \mathrm{CDCl}_{3}\right) \delta: 151.2,149.3$, 148.2, 143.9, 142.6, 142.5, 139.0, 138.9, 138.8, 137.4, 136.8, 136.7, 136.6, 134.3, 129.5, $129.4,129.4,129.2,128.6,128.3,125.4,123.7,123.3,118.5,21.2,21.1,20.2,20.0$. HRMS (MALDI-TOF) calcd. for $\mathrm{C}_{72} \mathrm{H}_{60} \mathrm{~B}_{4} \mathrm{Cl}_{2} \mathrm{~F}_{8} \mathrm{~N}_{8} \mathrm{~S}_{2}[\mathrm{M}]^{+}:$1366.4004, found 1366.4009 .
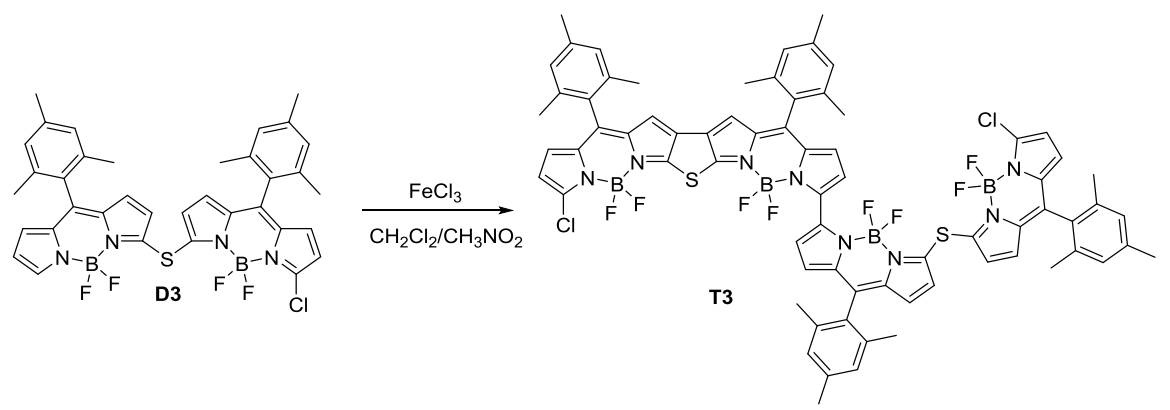

Synthesis of BODIPY T3: BODIPY T3 as a blackish solid was prepared in $24 \%$ yield (16 mg) from BODIPY D3 (68 mg, $0.1 \mathrm{mmol})$ and $\mathrm{FeCl}_{3}(324 \mathrm{mg}, 2 \mathrm{mmol})$ using the same procedure described for FD1. The crude product was purified through column 
chromatography on silica gel (eluent: petroleum ether/dichloromethane $=1: 1$ ). ${ }^{1} \mathrm{H}$ NMR (500 MHz, $\left.\mathrm{CDCl}_{3}\right) \delta: 7.66(\mathrm{~d}, J=4.5 \mathrm{~Hz}, 1 \mathrm{H}), 7.62(\mathrm{~d}, J=4.5 \mathrm{~Hz}, 1 \mathrm{H}), 6.98(\mathrm{~s}$, 2H), $6.96(\mathrm{~s}, 2 \mathrm{H}), 6.93(\mathrm{~d}, J=4.5 \mathrm{~Hz}, 4 \mathrm{H}), 6.71(\mathrm{~d}, J=4.5 \mathrm{~Hz}, 1 \mathrm{H}), 6.66(\mathrm{~d}, J=4.5 \mathrm{~Hz}$, $1 \mathrm{H}), 6.61(\mathrm{~d}, J=4.3 \mathrm{~Hz}, 1 \mathrm{H}), 6.59-6.51(\mathrm{~m}, 3 \mathrm{H}), 6.52-6.45(\mathrm{~m}, 4 \mathrm{H}), 6.33(\mathrm{t}, J=4.5 \mathrm{~Hz}$, 2H), 2.38 (s, 3H), $2.36(\mathrm{~s}, 3 \mathrm{H}), 2.34(\mathrm{~s}, 3 \mathrm{H}), 2.33(\mathrm{~s}, 3 \mathrm{H}), 2.18(\mathrm{~s}, 6 \mathrm{H}), 2.16(\mathrm{~s}, 6 \mathrm{H})$, $2.10(\mathrm{~s}, 6 \mathrm{H}), 2.08(\mathrm{~s}, 6 \mathrm{H}) .{ }^{13} \mathrm{C} \mathrm{NMR}\left(126 \mathrm{MHz}, \mathrm{CDCl}_{3}\right) \delta: 163.4,162.6,151.5,150.0$, $148.4,147.8,144.4,143.4,143.4,143.0,143.0,140.8,140.2,139.6,139.5,139.4,139.3$, 139.2, 137.8, 137.3, 137.2, 137.1, 137.1, 134.8, 129.9, 129.7, 129.6, 129.1, 129.0, 128.9, $128.7,127.4,127.2,125.4,124.1,123.7,119.0,118.5,118.3,117.7,21.5,21.5,20.6$, 20.6, 20.4, 20.3. HRMS (MALDI-TOF) calcd. for $\mathrm{C}_{72} \mathrm{H}_{58} \mathrm{~B}_{4} \mathrm{Cl}_{2} \mathrm{~F}_{8} \mathrm{~N}_{8} \mathrm{~S}_{2}[\mathrm{M}]^{+}:$: 364.3847 , found 1364.3852 .
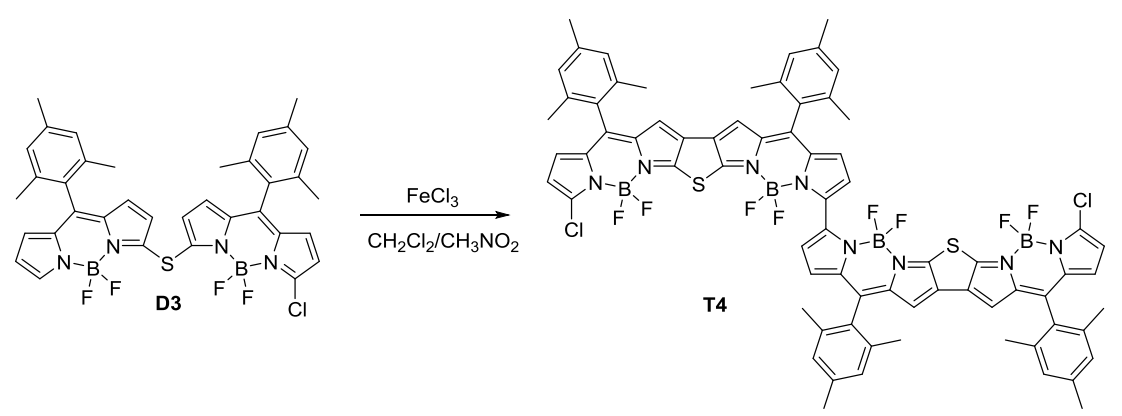

Synthesis of BODIPY T4: BODIPY T4 as a blackish solid was prepared in $36 \%$ yield (24 mg) from BODIPY D3 (68 mg, $0.1 \mathrm{mmol}$ ) and $\mathrm{FeCl}_{3}$ (486 mg, $5 \mathrm{mmol}$ ) using the same procedure described for FD1. The crude product was purified through column chromatography on silica gel (eluent: petroleum ether/dichloromethane $=2: 1$ ). ${ }^{1} \mathrm{H}$ NMR $\left(500 \mathrm{MHz}, \mathrm{CDCl}_{3}\right) \delta: 7.61(\mathrm{~d}, J=4.5 \mathrm{~Hz}, 2 \mathrm{H}), 6.98(\mathrm{~s}, 4 \mathrm{H}), 6.94(\mathrm{~s}, 4 \mathrm{H}), 6.69$ (d, $J=$ $4.5 \mathrm{~Hz}, 2 \mathrm{H}), 6.54(\mathrm{~d}, J=4.2 \mathrm{~Hz}, 2 \mathrm{H}), 6.50(\mathrm{~s}, 2 \mathrm{H}), 6.48(\mathrm{~s}, 2 \mathrm{H}), 6.32(\mathrm{~d}, J=4.2 \mathrm{~Hz}$, 2H), 2.38 (s, 6H), 2.34 (s, 6H), 2.18 (s, 12H), 2.10 (s, 12H). $\left.{ }^{13} \mathrm{C} \mathrm{NMR} \mathrm{(126} \mathrm{MHz,} \mathrm{CDCl}_{3}\right)$ $\delta: 163.4,162.7,147.5,143.5,143.4,140.8,140.2$, 139.6, 139.5, 139.3, 137.3, 137.1, $134.8,129.7,128.8,128.7,127.3,127.2,124.9,118.5,118.4,117.8,21.6,21.5,20.6$, 20.3. HRMS (MALDI-TOF) calcd. for $\mathrm{C}_{72} \mathrm{H}_{56} \mathrm{~B}_{4} \mathrm{Cl}_{2} \mathrm{~F}_{8} \mathrm{~N}_{8} \mathrm{~S}_{2}[\mathrm{M}]^{+}:$1362.3691, found 1362.3697. 


\section{Photophysical data}

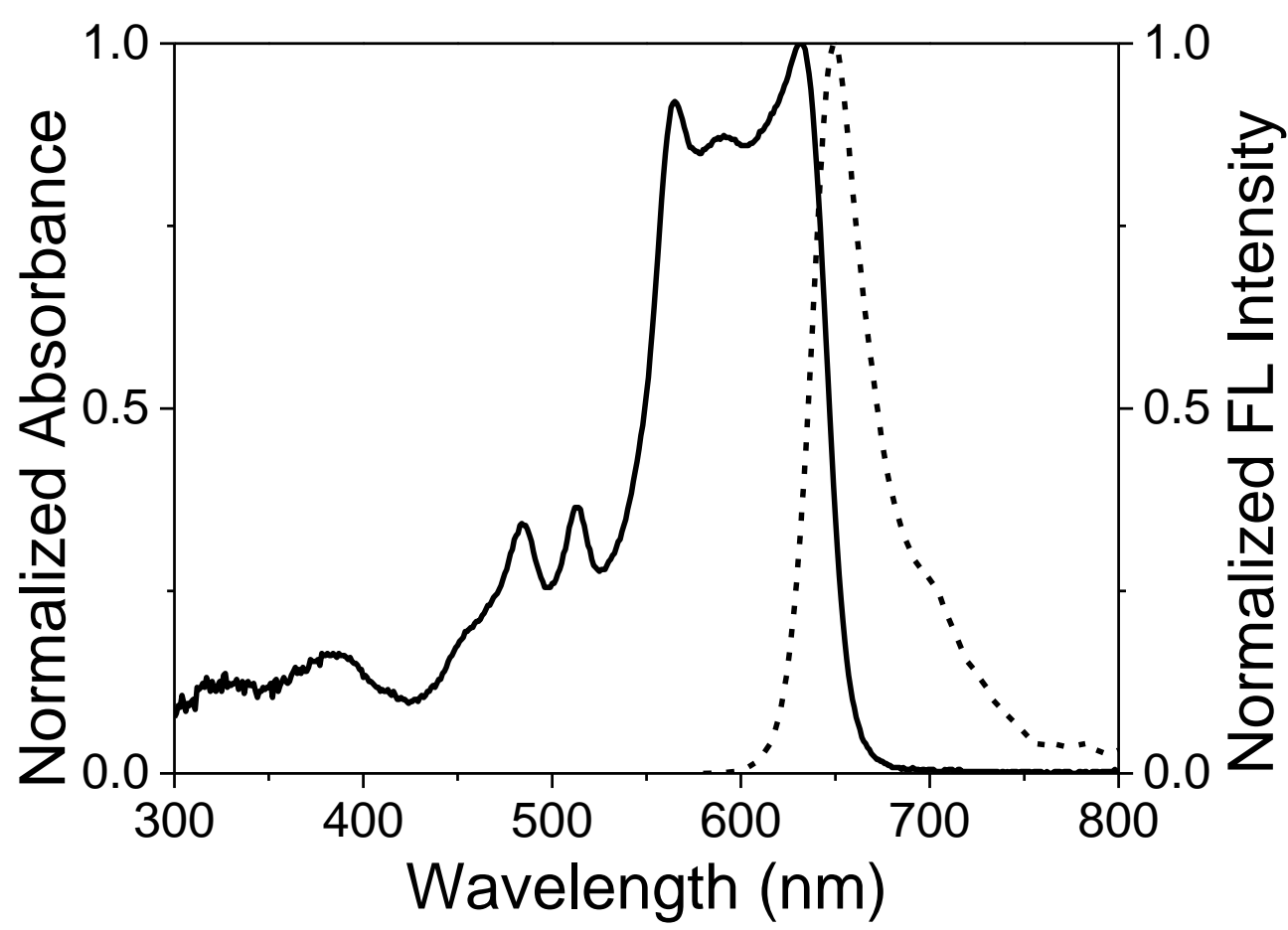

Figure S2. Normalized absorption (solid line) and emission (dashed line) spectra of BODIPY D1 recorded in toluene, excited at $570 \mathrm{~nm}$.

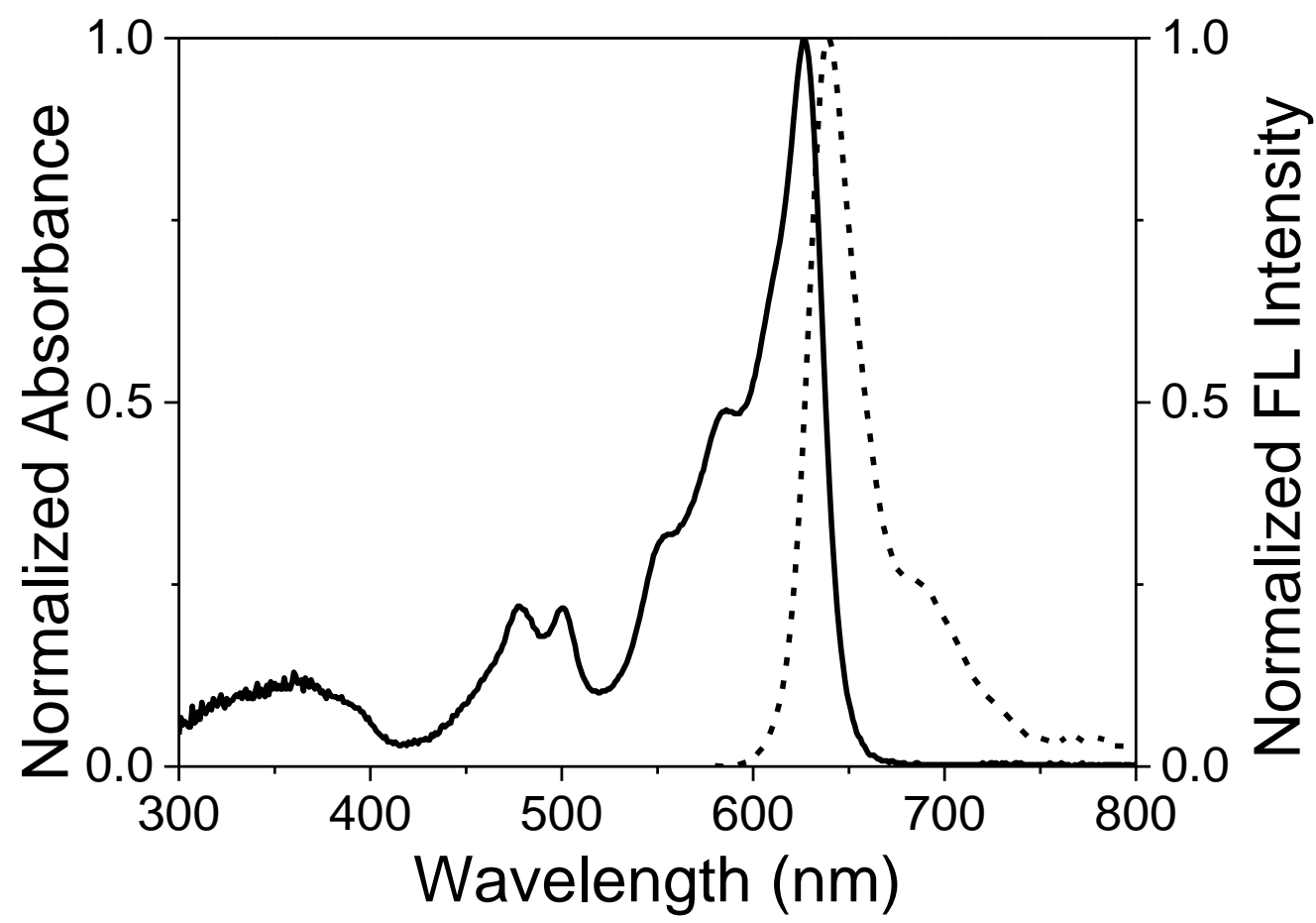

Figure S3. Normalized absorption (solid line) and emission (dashed line) spectra of BODIPY D2 recorded in toluene, excited at $570 \mathrm{~nm}$. 


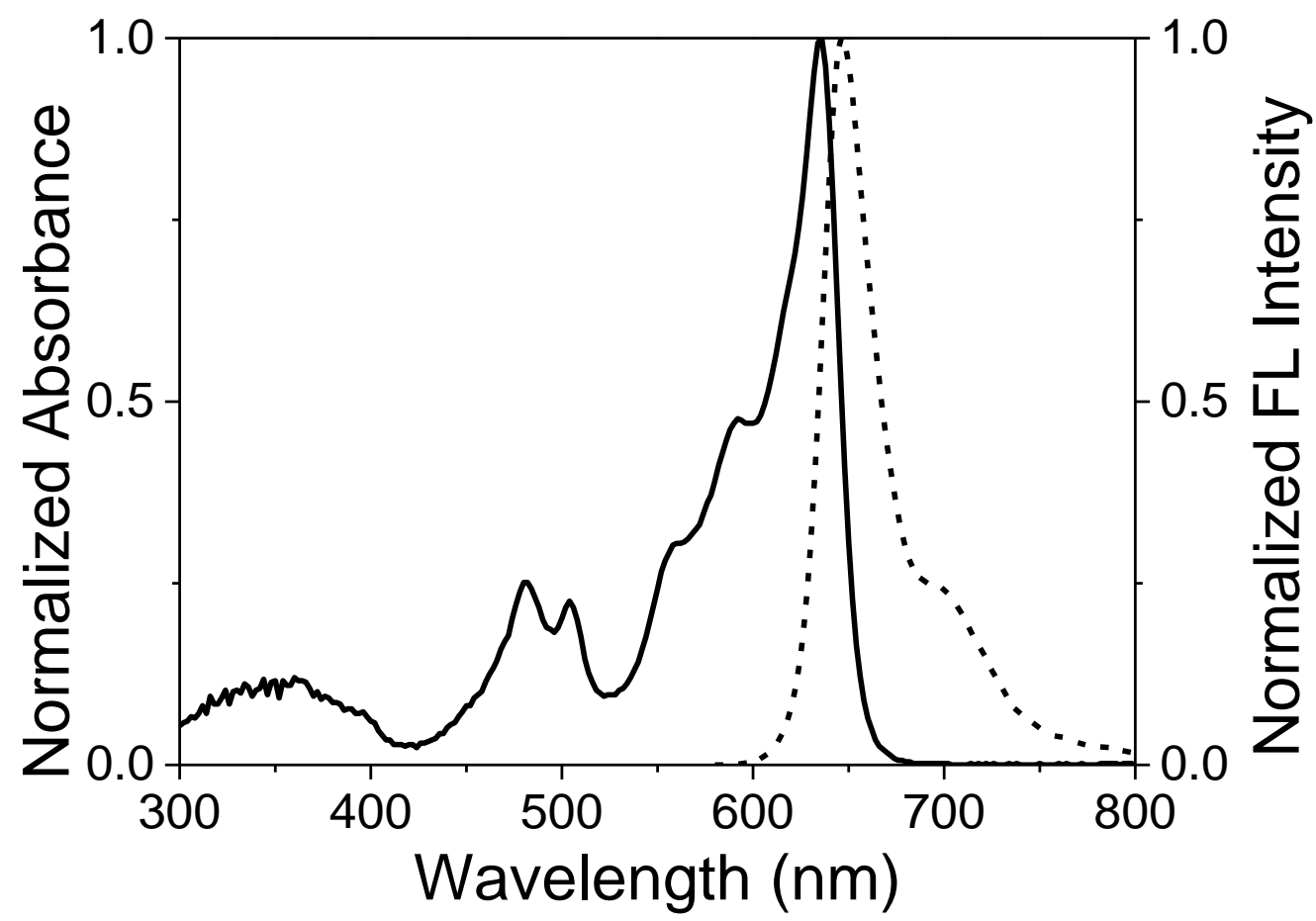

Figure S4. Normalized absorption (solid line) and emission (dashed line) spectra of BODIPY D3 recorded in toluene, excited at $570 \mathrm{~nm}$.

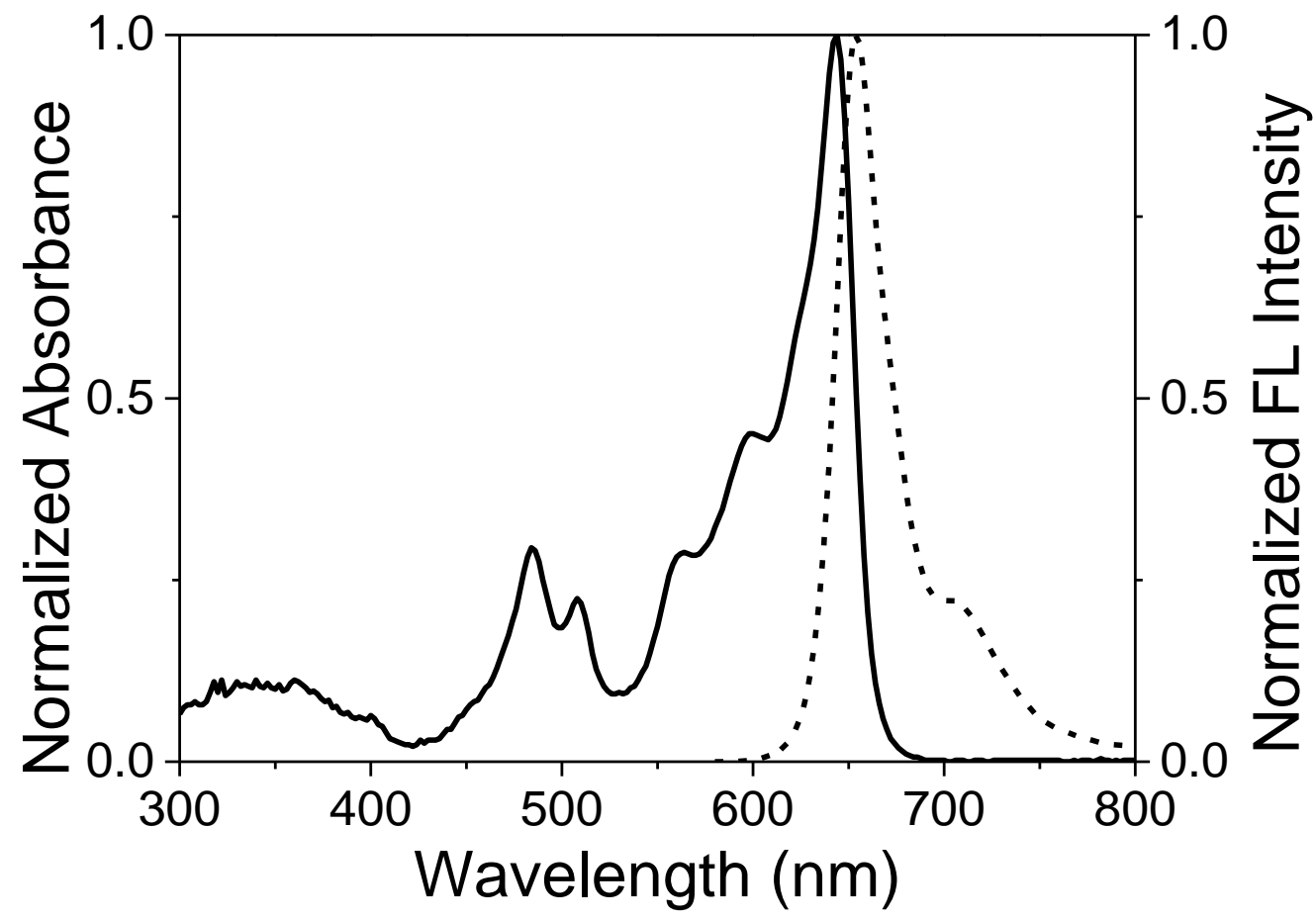

Figure S5. Normalized absorption (solid line) and emission (dashed line) spectra of BODIPY D4 recorded in toluene, excited at $570 \mathrm{~nm}$. 


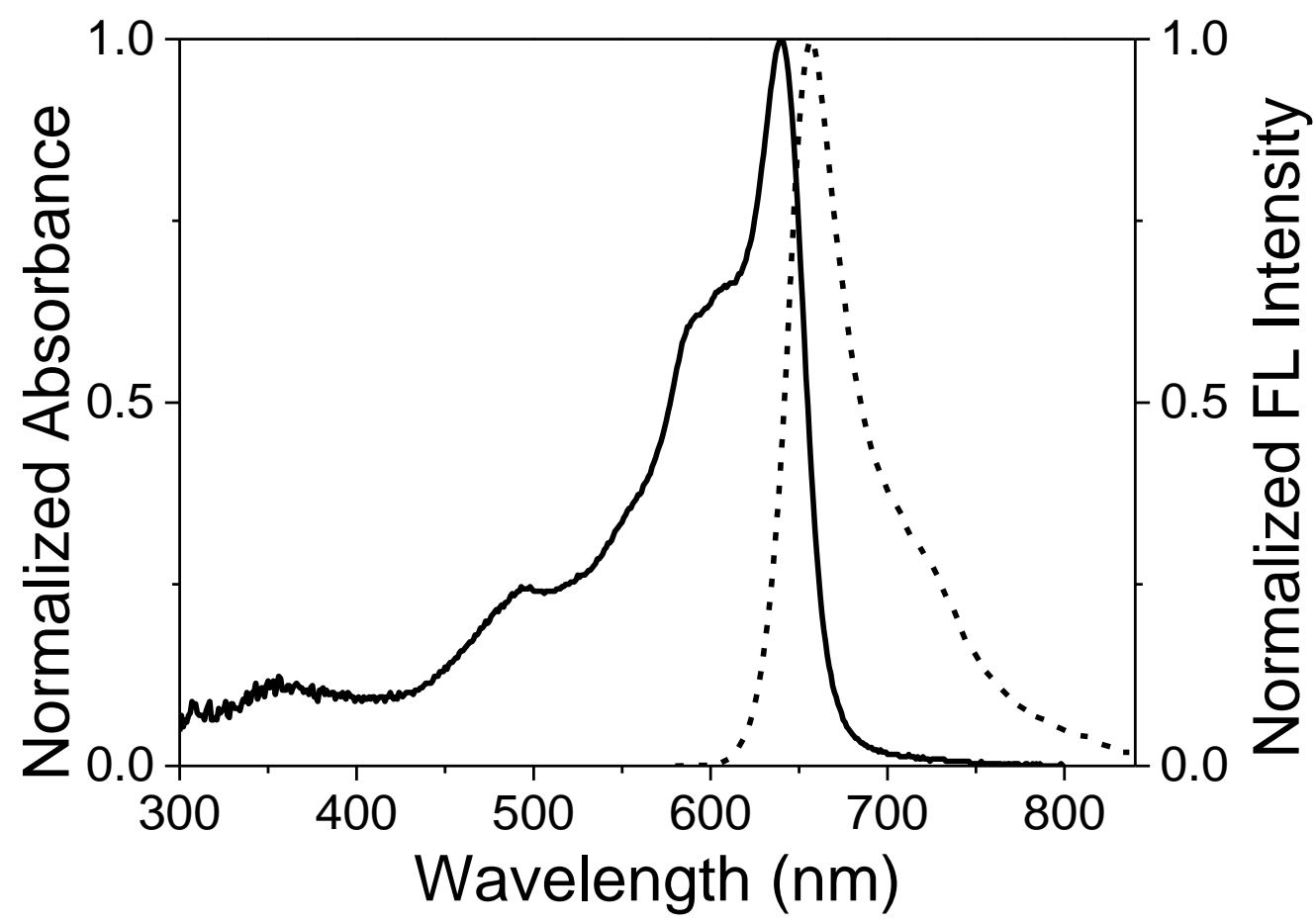

Figure S6. Normalized absorption (solid line) and emission (dashed line) spectra of BODIPY FD1 recorded toluene, excited at $570 \mathrm{~nm}$.

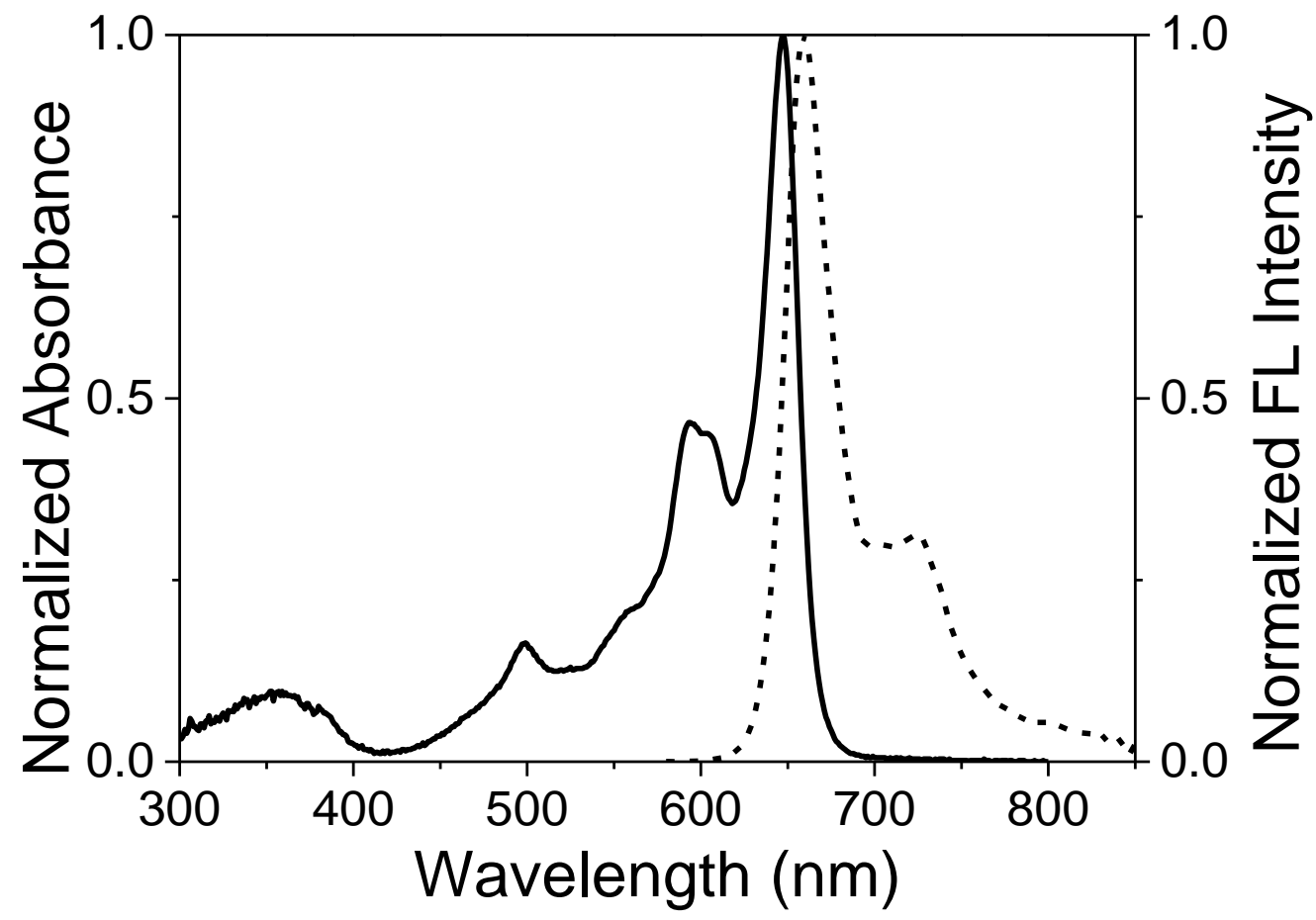

Figure S7. Normalized absorption (solid line) and emission (dashed line) spectra of BODIPY FD2 recorded in toluene, excited at $570 \mathrm{~nm}$. 


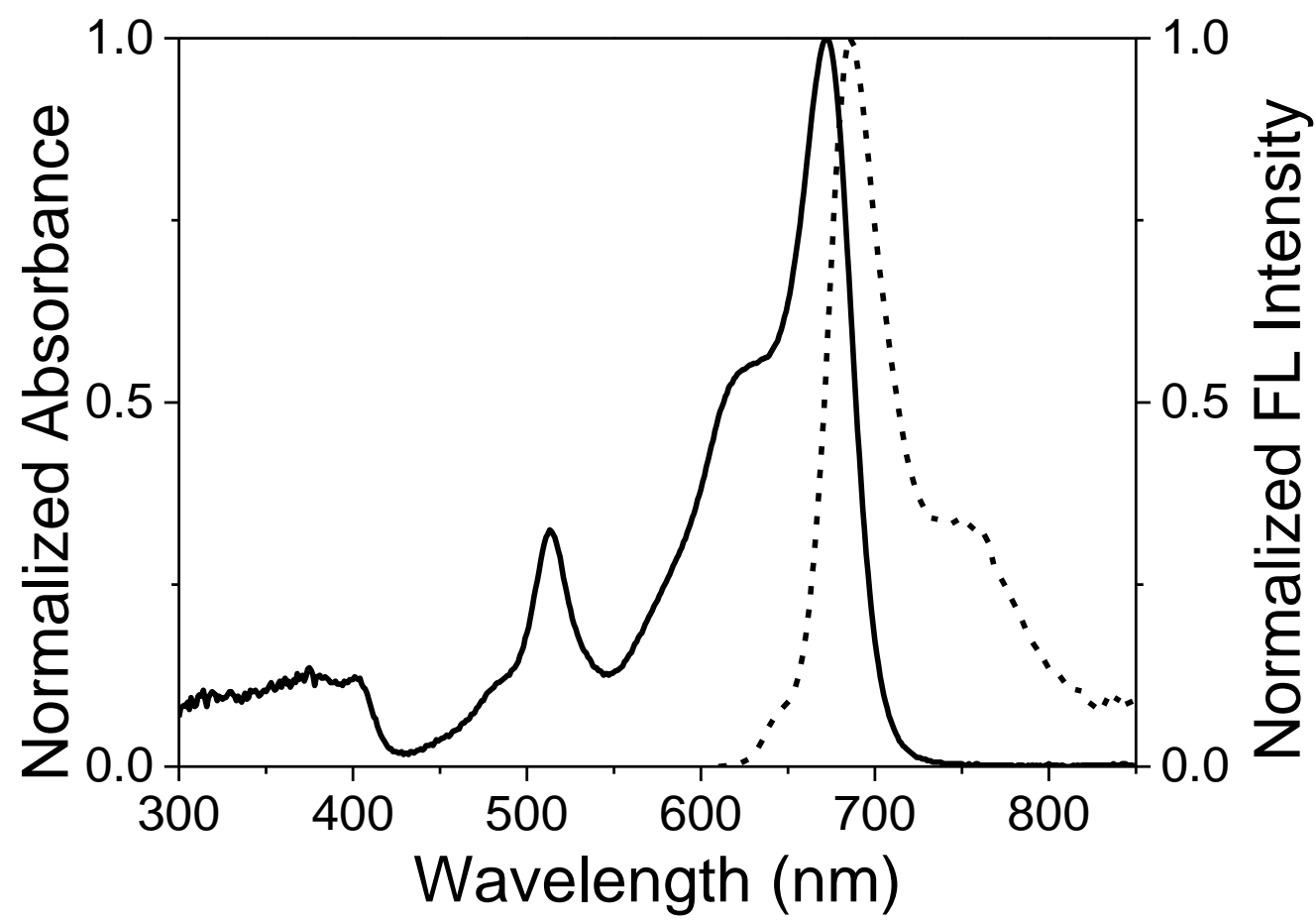

Figure S8. Normalized absorption (solid line) and emission (dashed line) spectra of BODIPY FD3 recorded in toluene, excited at $610 \mathrm{~nm}$.

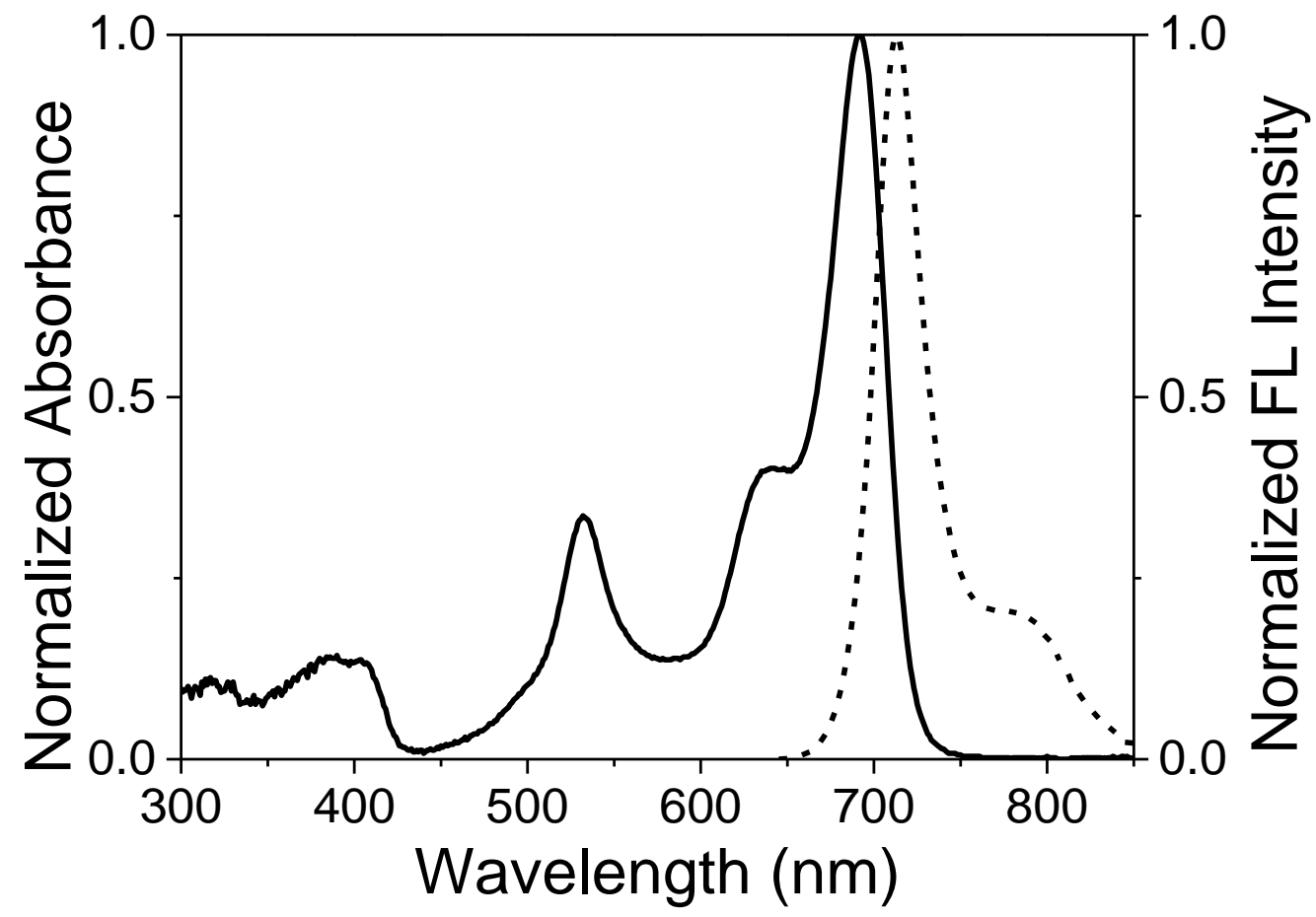

Figure S9. Normalized absorption (solid line) and emission (dashed line) spectra of BODIPY FD4 recorded in toluene, excited at $640 \mathrm{~nm}$. 


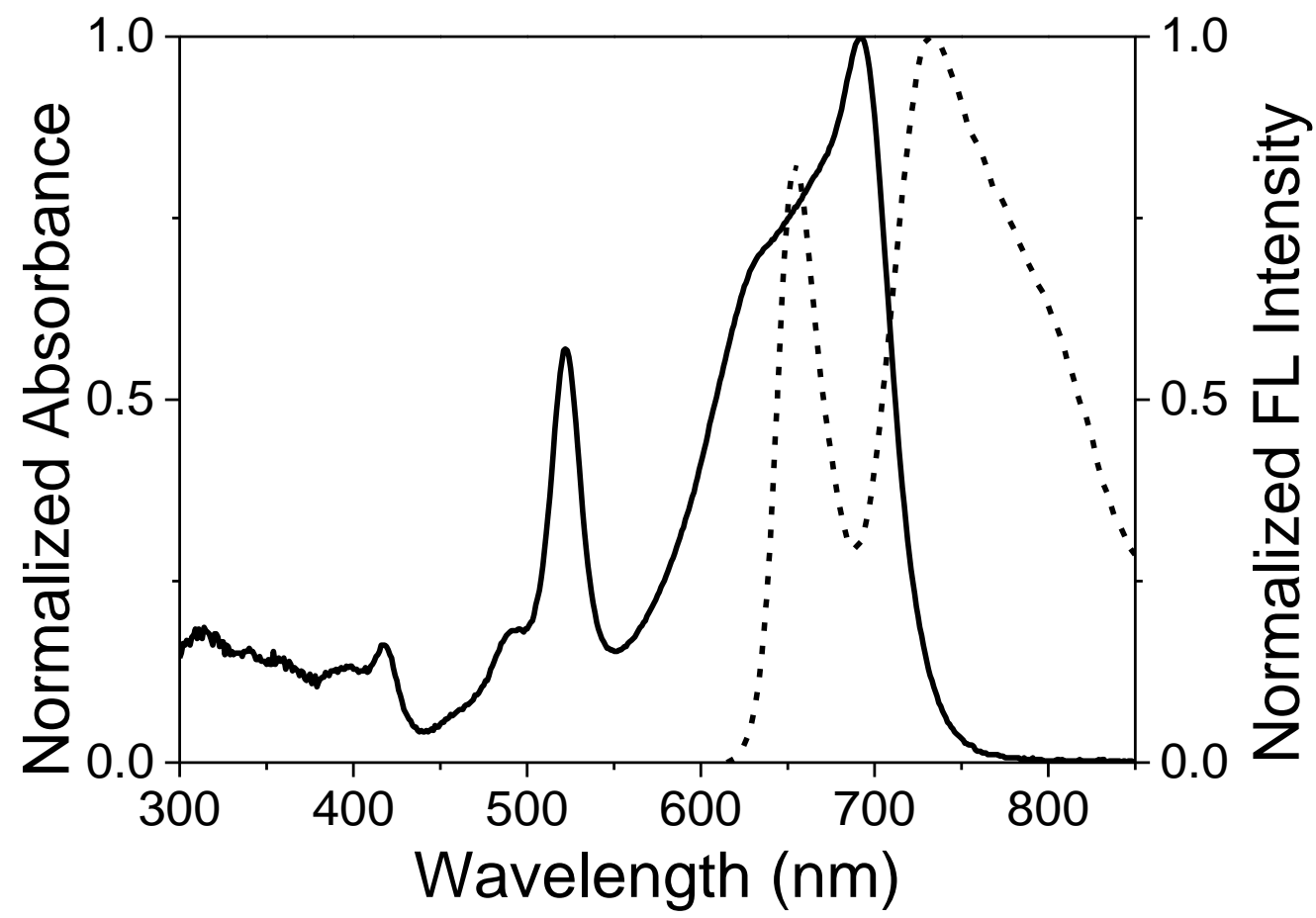

Figure S10. Normalized absorption (solid line) and emission (dashed line) spectra of BODIPY FD5 recorded in toluene, excited at $610 \mathrm{~nm}$.

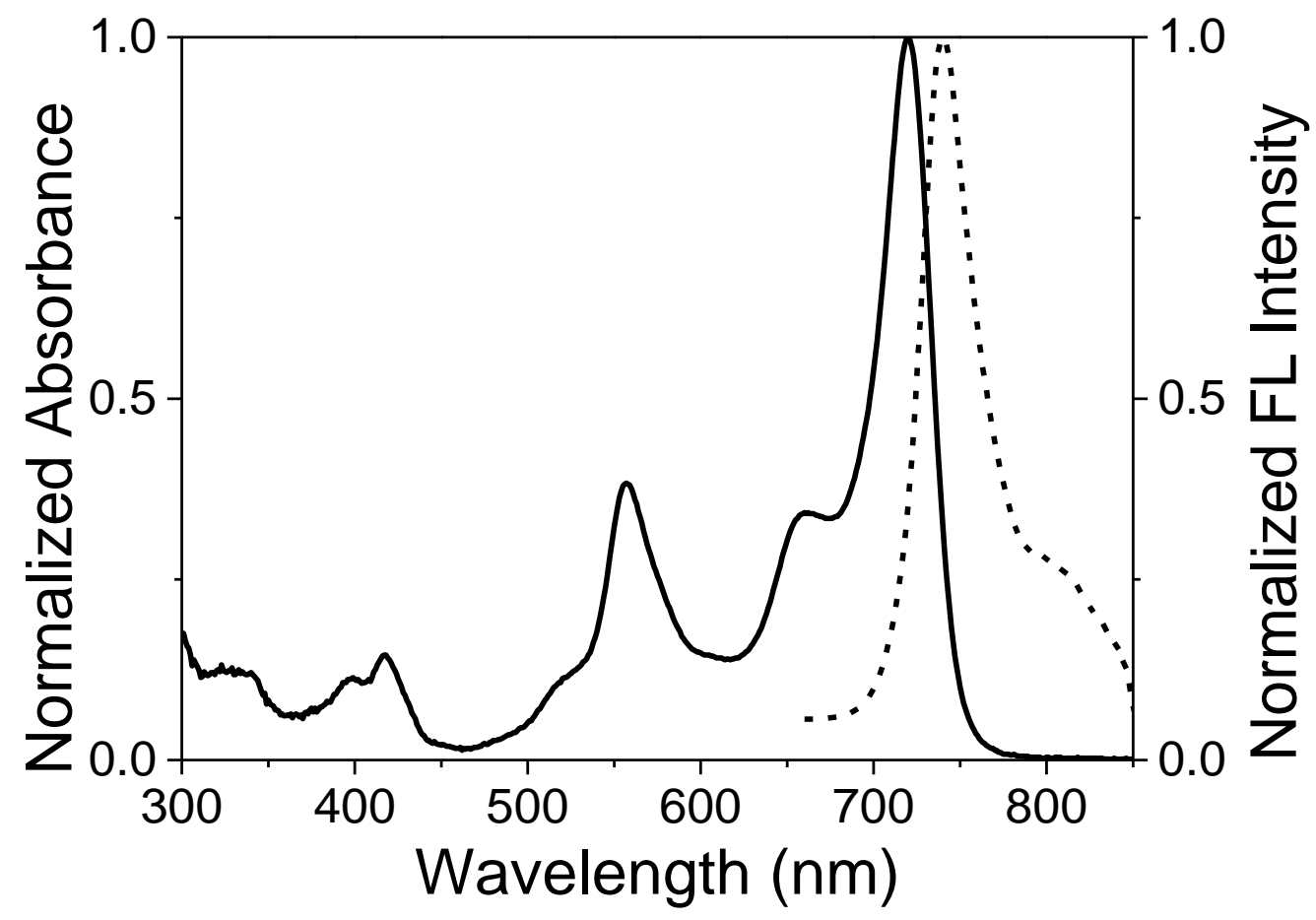

Figure S11. Normalized absorption (solid line) and emission (dashed line) spectra of BODIPY FD6 recorded in toluene, excited at $650 \mathrm{~nm}$. 


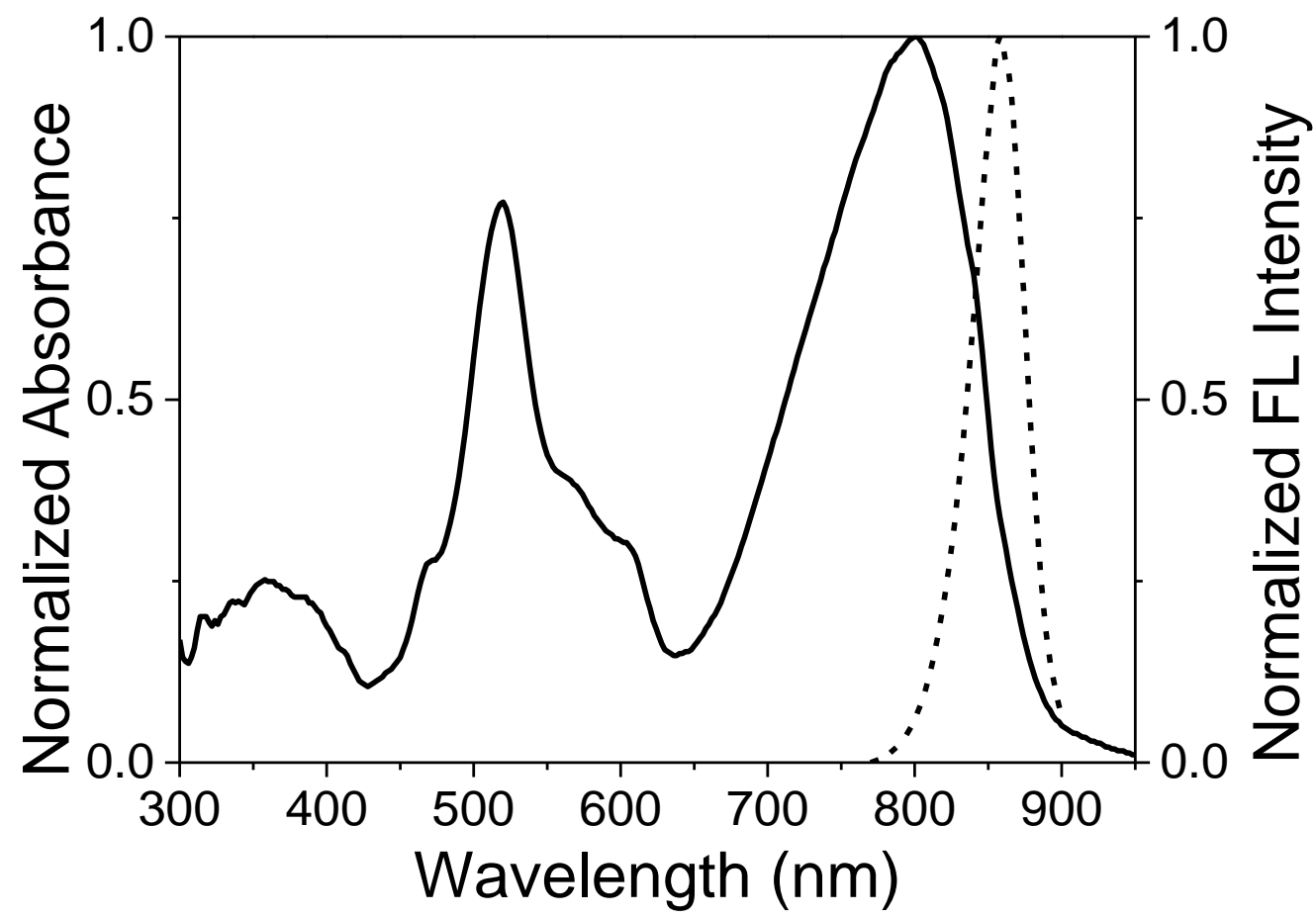

Figure S12. Normalized absorption (solid line) and emission (dashed line) spectra of BODIPY T1 recorded in toluene, excited at $750 \mathrm{~nm}$.

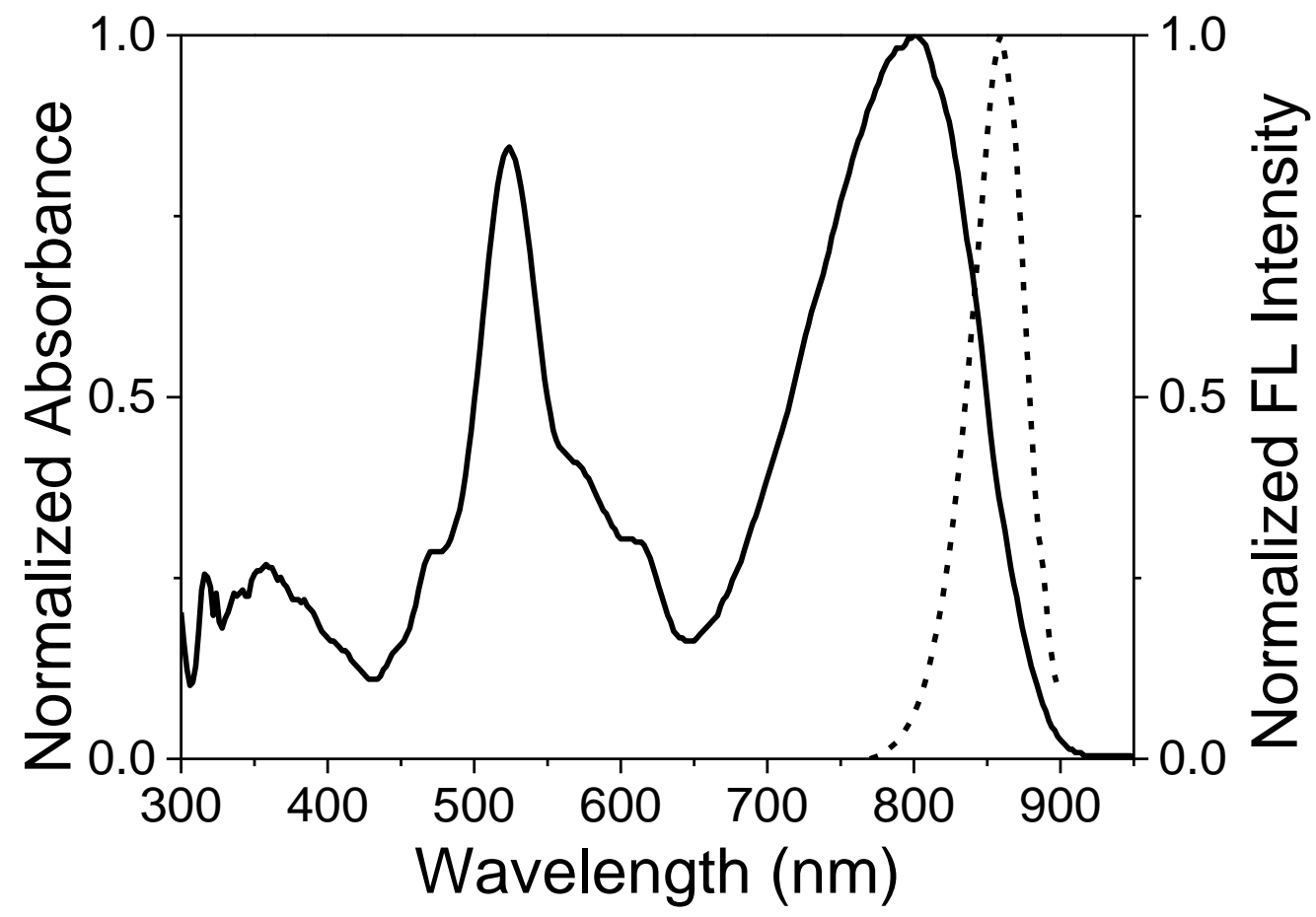

Figure S13. Normalized absorption (solid line) and emission (dashed line) spectra of BODIPY T2 recorded in toluene, excited at $750 \mathrm{~nm}$. 


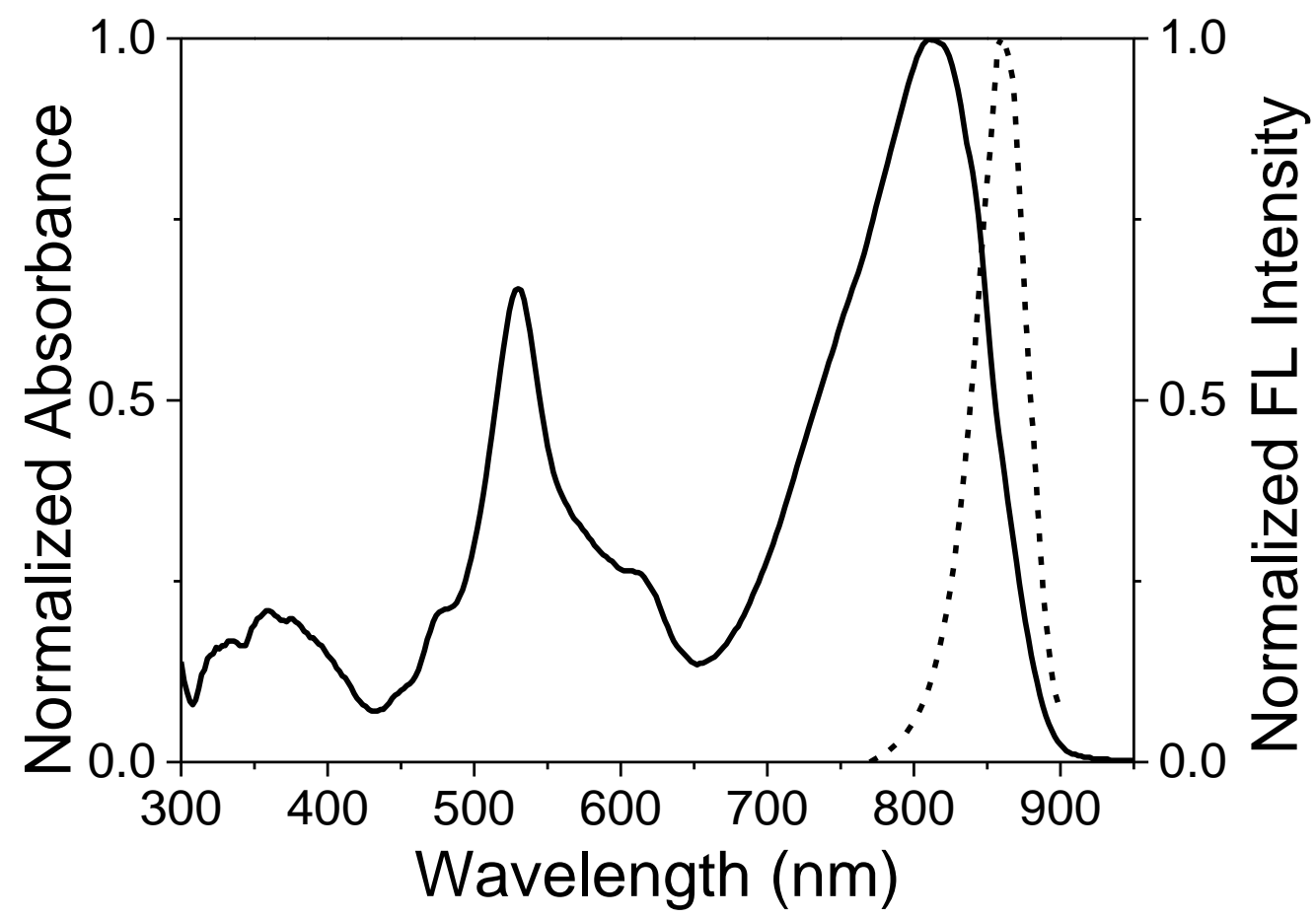

Figure S14. Normalized absorption (solid line) and emission (dashed line) spectra of BODIPY T3 recorded in toluene, excited at $750 \mathrm{~nm}$.

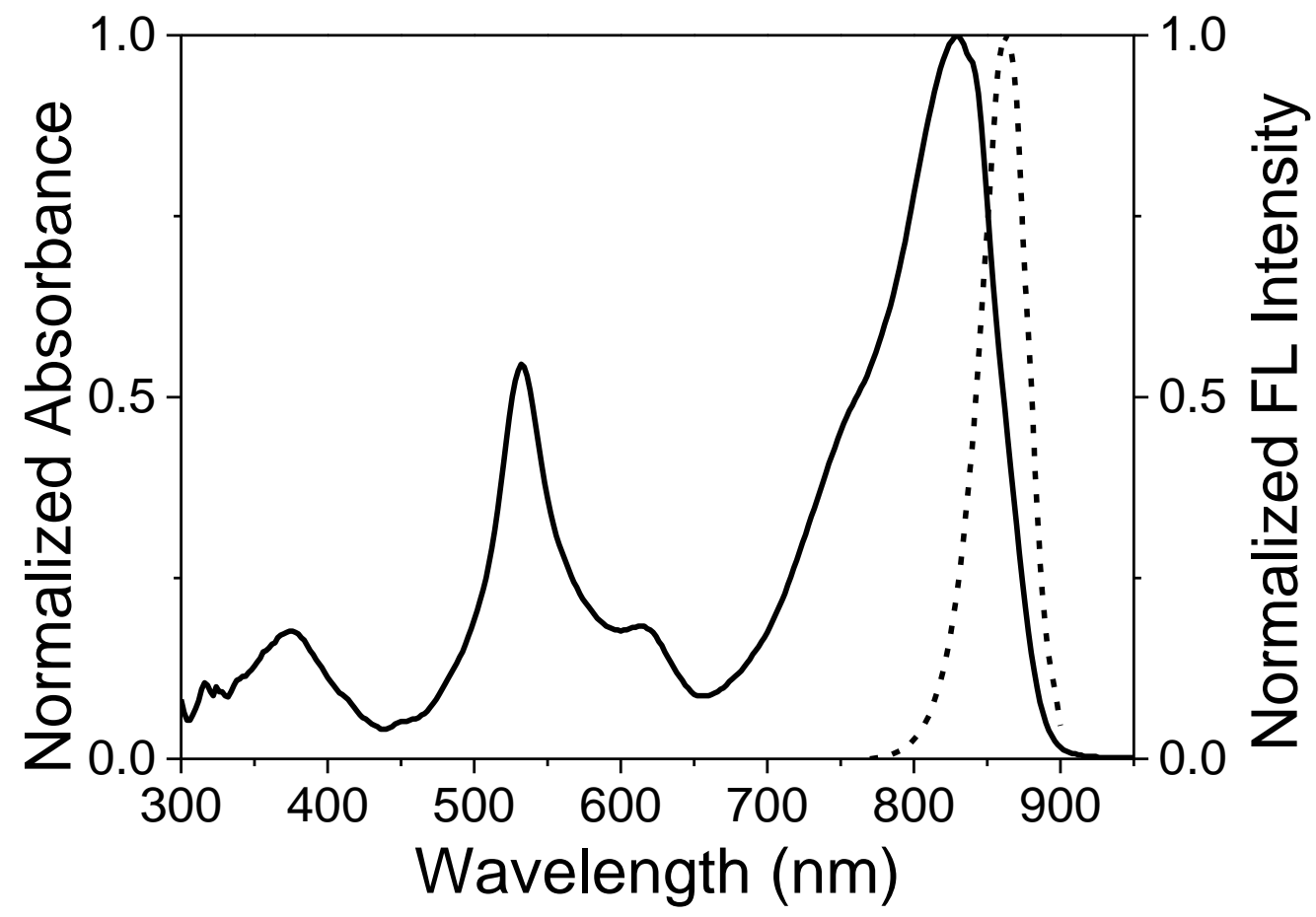

Figure S15. Normalized absorption (solid line) and emission (dashed line) spectra of BODIPY T4 recorded in toluene, excited at $750 \mathrm{~nm}$. 


\section{Electrochemical spectra}

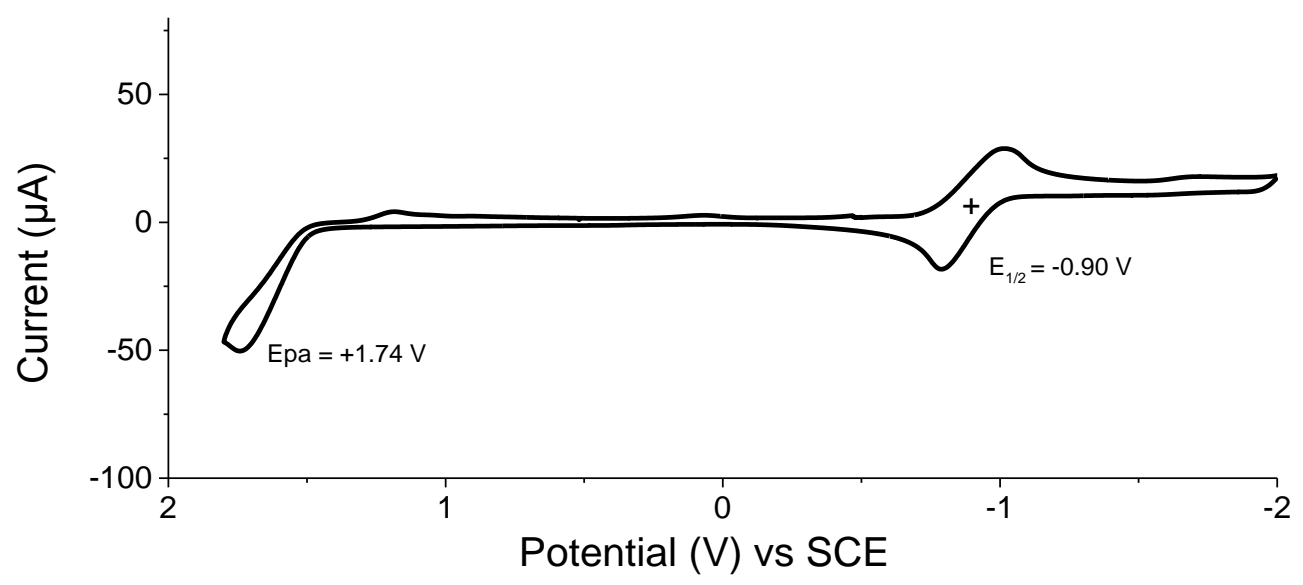

Figure S16. Cyclic voltammograms of $3 \mathrm{mM}$ M2 measured in dichloromethane solution, containing $0.1 \mathrm{M} \mathrm{TBAPF}_{6}$ as the supporting electrolyte at room temperature. Glassy carbon electrode as a working electrode, and the scan rate at $100 \mathrm{mV} \mathrm{s}^{-1}$.

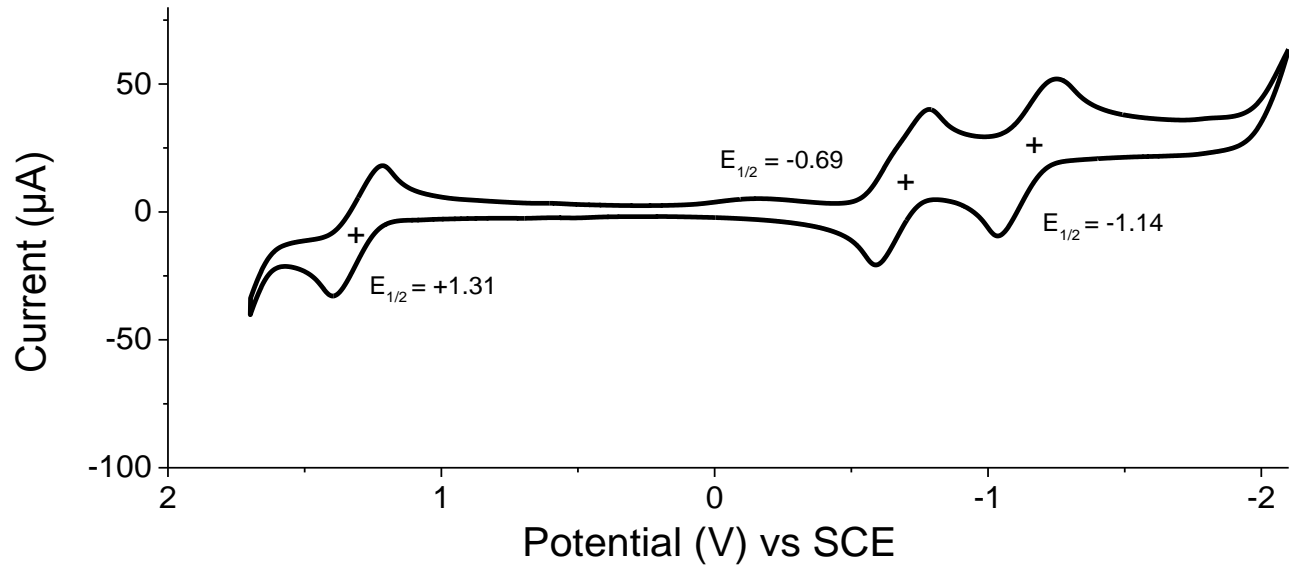

Figure S17. Cyclic voltammograms of $2 \mathrm{mM} \mathrm{D4}$ measured in dichloromethane solution, containing $0.1 \mathrm{M} \mathrm{TBAPF}_{6}$ as the supporting electrolyte at room temperature. Glassy carbon electrode as a working electrode, and the scan rate at $100 \mathrm{mV} \mathrm{s}^{-1}$. 


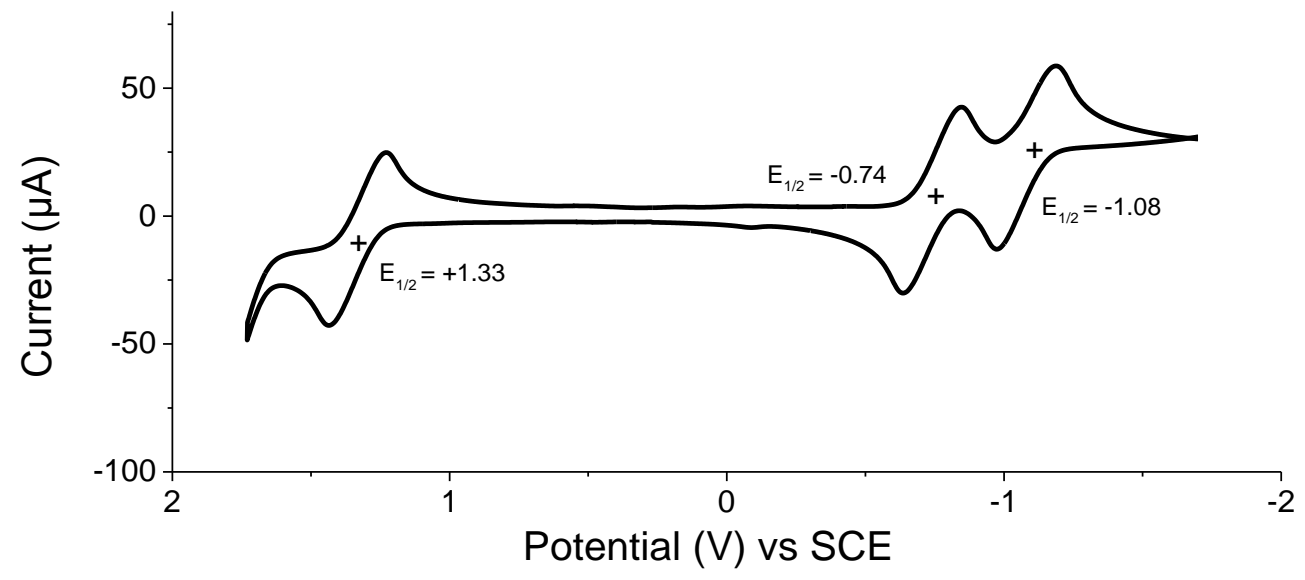

Figure S18. Cyclic voltammograms of $2 \mathrm{mM}$ FD2 measured in dichloromethane solution, containing $0.1 \mathrm{M} \mathrm{TBAPF}_{6}$ as the supporting electrolyte at room temperature. Glassy carbon electrode as a working electrode, and the scan rate at $100 \mathrm{mV} \mathrm{s}^{-1}$.

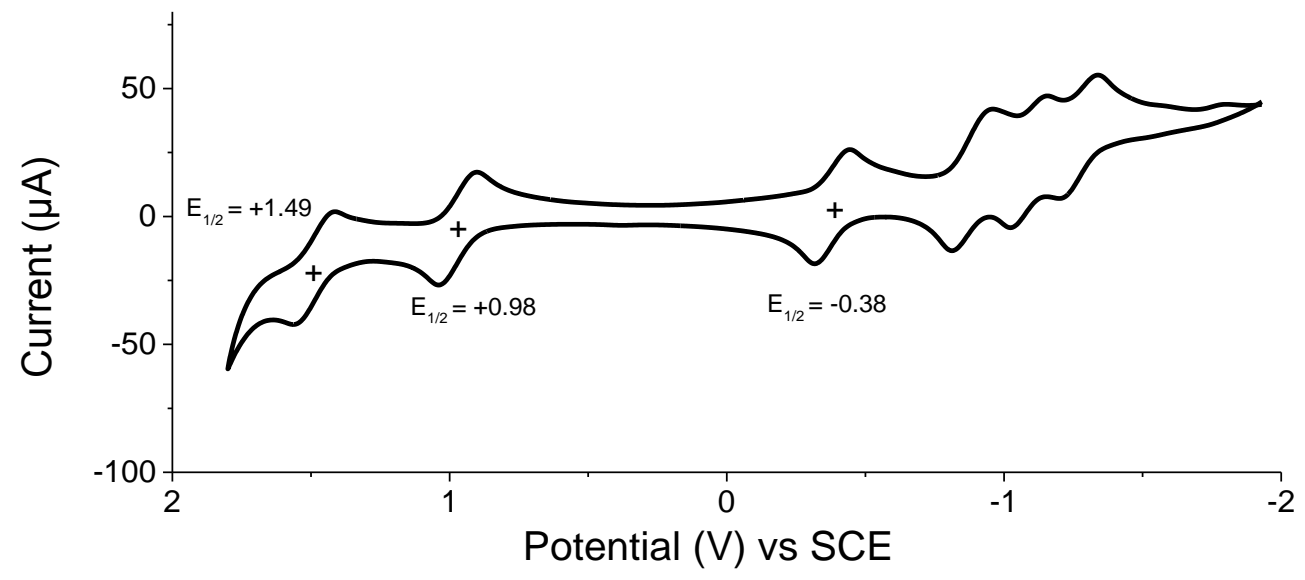

Figure S19. Cyclic voltammograms of $2 \mathrm{mM}$ T2 measured in dichloromethane solution, containing $0.1 \mathrm{M} \mathrm{TBAPF}_{6}$ as the supporting electrolyte at room temperature. Glassy carbon electrode as a working electrode, and the scan rate at $100 \mathrm{mV} \mathrm{s}^{-1}$. 


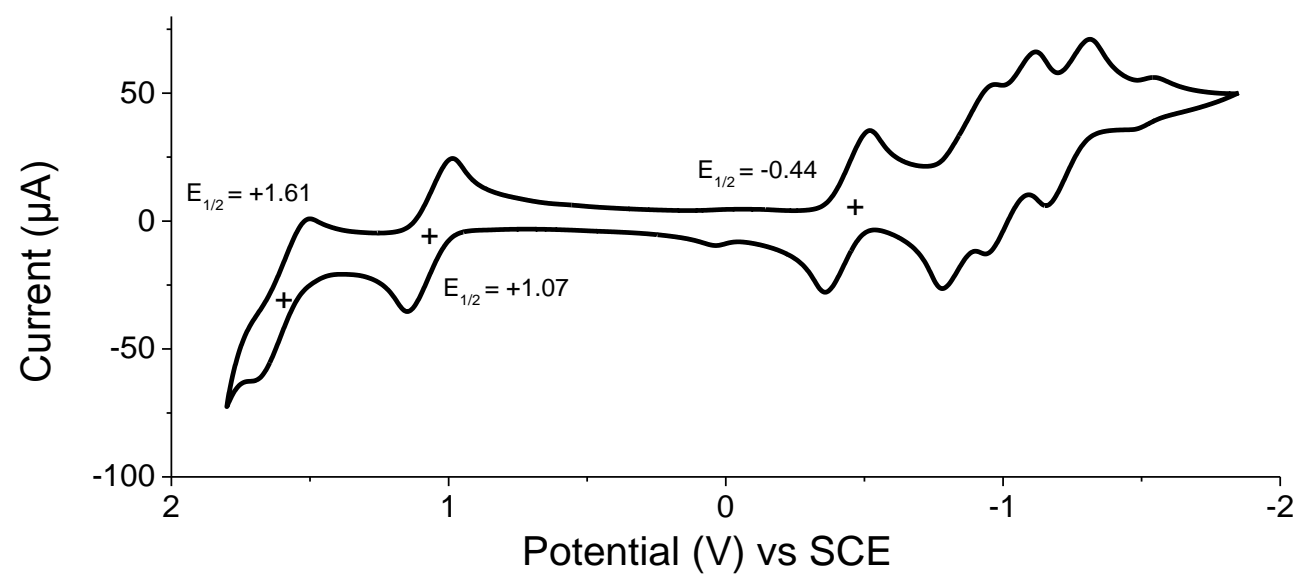

Figure S20. Cyclic voltammograms of $2 \mathrm{mM}$ T4 measured in dichloromethane solution, containing $0.1 \mathrm{M} \mathrm{TBAPF}_{6}$ as the supporting electrolyte at room temperature. Glassy carbon electrode as a working electrode, and the scan rate at $100 \mathrm{mV} \mathrm{s}^{-1}$.

Table S3. Electrochemical data acquired at $100 \mathrm{mV} / \mathrm{s}$, and HOMO-LUMO Gaps determined from spectroscopy of dyes M2, D4, FD2, T2 and T4.

\begin{tabular}{cccccccccc}
\hline dyes & $E_{1 / 2}{ }^{\text {red }}(\mathrm{V})$ & $E_{1 / 2}{ }^{\mathrm{xx}}(\mathrm{V})$ & $\begin{array}{c}E_{\mathrm{pa}} \\
(\mathrm{V})\end{array}$ & $\begin{array}{c}E_{\text {red }}{ }^{\text {onset }} \\
(\mathrm{V})\end{array}$ & $\begin{array}{c}E_{\mathrm{ox}}{ }^{\text {onset }} \\
(\mathrm{V})\end{array}$ & $\begin{array}{c}\mathrm{LUMO} \\
(\mathrm{eV})\end{array}$ & $\begin{array}{c}\mathrm{HOMO} \\
(\mathrm{eV})\end{array}$ & $\begin{array}{c}E_{\mathrm{g}}^{\mathrm{e}} \\
(\mathrm{eV})\end{array}$ & $\begin{array}{c}E_{\mathrm{g}}{ }^{\mathrm{e}} \\
(\mathrm{eV})\end{array}$ \\
\hline M2 & -0.90 & - & 1.74 & -0.73 & 1.51 & -3.67 & -5.91 & 2.24 & 2.28 \\
D4 & $-0.69,-1.14$ & 1.13 & - & -0.54 & 1.22 & -3.86 & -5.62 & 1.76 & 1.85 \\
FD2 & $-0.74,-1.08$ & 1.33 & - & -0.67 & 1.24 & -3.73 & -5.64 & 1.91 & 1.87 \\
& $-0.38,-0.88$, & 1.49 & - & & & & & & \\
T2 & $-1.09,-1.28$ & 0.98 & - & -0.29 & 0.91 & -4.11 & -5.31 & 1.20 & 1.38 \\
& $-0.44,-0.87$, & 1.61 & - & -0.37 & 1.01 & -4.03 & -5.41 & 1.38 & 1.41 \\
T4 & $-1.03,-1.24$ & 1.07 & & & & & & & \\
\hline
\end{tabular}

$E_{1 / 2}{ }^{\text {red }}=$ half wave potentials of reversible reduction potential; $E_{1 / 2}{ }^{\mathrm{ox}}=$ half wave potentials of reversible oxidation potential; $E_{\mathrm{pa}}=$ irreversible oxidation peak potentials; $E_{\mathrm{red}}{ }^{\text {onset }}=$ the onset reduction potentials; $E_{\mathrm{ox}}{ }^{\text {onset }}=$ the onset oxidation potentials; $E_{\mathrm{LUMO}}=-\mathrm{e}\left(E_{\mathrm{red}}{ }^{\text {onset }}+4.4\right) ; E_{\mathrm{HOMO}}=$ $-\mathrm{e}\left(E_{\mathrm{ox}}{ }^{\text {onset }}+4.4\right) ; E_{\mathrm{g}}{ }^{\mathrm{e}}=$ bandgap, obtained from the intercept of the electrochemical data; $E_{\mathrm{g}}{ }^{\mathrm{e}}=$ $E_{\mathrm{LUMO}}-E_{\mathrm{HOMO}} ; E_{\mathrm{g}}{ }^{\mathrm{o}}=$ bandgap, obtained from the intercept of the absorption spectra. 


\section{DFT calculations}

The ground state geometry was optimized by using DFT method at B3LYP/6-31G(d) level. The same method was used for vibrational analysis to verify that the optimized structures correspond to local minima on the energy surface. TD-DFT computations were used the optimized ground state geometries under the B3LYP/6-31+G(d,p) theoretical level. NICS(0) values were calculated at the GIAO-B3lyp/6-31+G(d,p) level. The calculated molecules in dichloromethane were done using the Self Consistent Reaction Field (SCRF) method and Polarizable Continuum Model (PCM). All of the calculations were carried out by the methods implemented in Gaussian 09 package. ${ }^{9}$
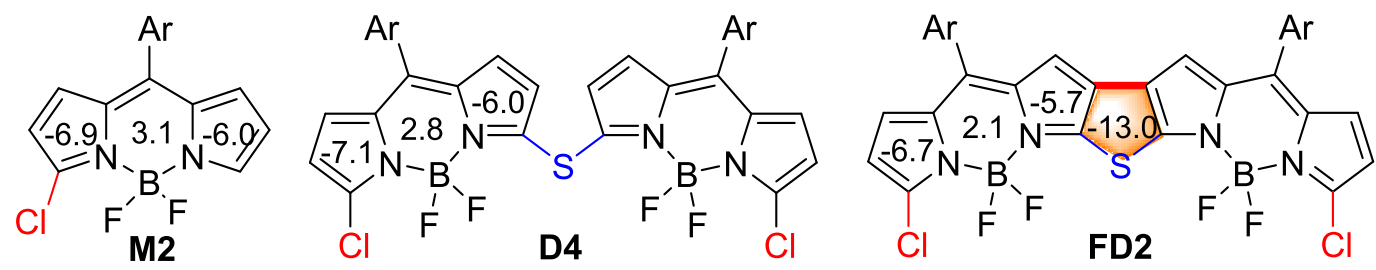

Figure S21. NICS(0) values (ppm) for M2, D4 and FD2, calculated at the B3LYP/631+G(d,p)//B3LYP/6- 31G(d) level.

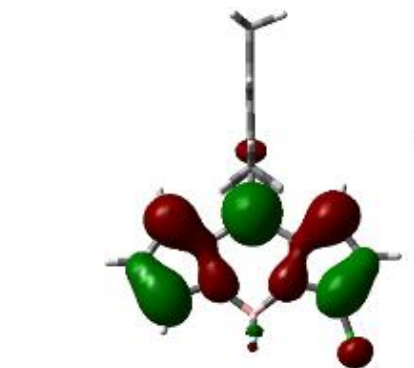

LUMO $-2.93 \mathrm{eV}$

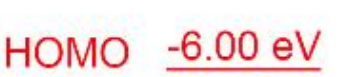

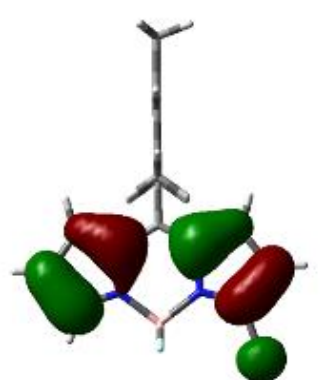

M2

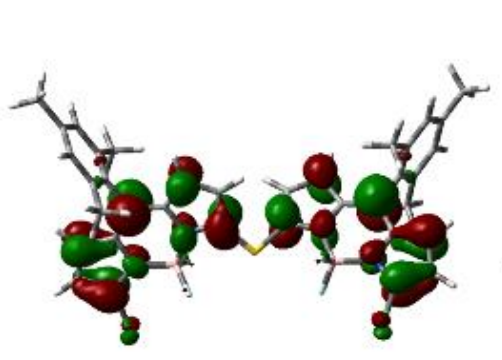

$\underline{-3.16 \mathrm{eV}}$

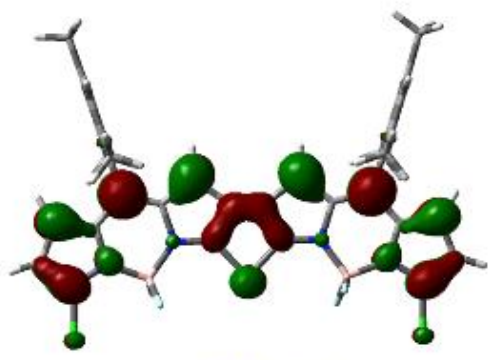

$\underline{-3.08 \mathrm{eV}}$ $\underline{-5.74 \mathrm{eV}}$

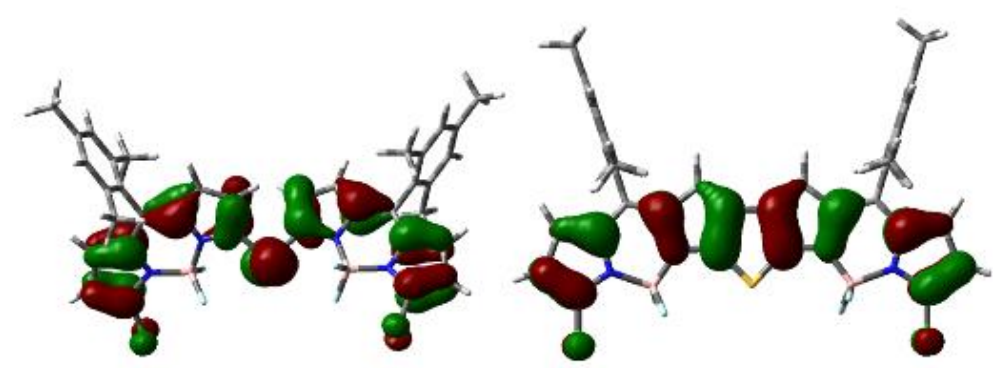

FD2

Figure S22. Pictorial presentation of LUMO, HOMO and their energy levels for M2, D4 and FD2. 
Table S4. Selected electronic excitation energies (eV) and oscillator strengths (f), configurations of the low-lying excited states of the M2, D4 and FD2 calculated by TDDFT//B3LYP/6-31+G(d,p), based on the optimized ground state geometries. The TDDFT of all the molecules in dichloromethane were using the Self Consistent Reaction Field (SCRF) method and the Polarizable Continuum Model (PCM).

\begin{tabular}{|c|c|c|c|c|c|}
\hline & \multirow{2}{*}{$\begin{array}{l}\text { Electronic } \\
\text { transition }\end{array}$} & \multicolumn{4}{|c|}{ TD//B3LYP/6-31G(d, p) } \\
\hline & & Energy/ eV ${ }^{[a]}$ & $f^{[\mathrm{b}]}$ & Composition $^{[\mathrm{c}]}$ & $\mathrm{CI}^{[\mathrm{d}]}$ \\
\hline \multirow[t]{5}{*}{ M2 } & $\mathrm{S} 0 \rightarrow \mathrm{S} 1$ & $2.8187 \mathrm{eV} 439.86 \mathrm{~nm}$ & 0.0001 & HOMO $-1 \rightarrow$ LUMO & 0.7050 \\
\hline & $\mathrm{S} 0 \rightarrow \mathrm{S} 2$ & $2.8765 \mathrm{eV} 431.03 \mathrm{~nm}$ & 0.5300 & HOMO $-3 \rightarrow$ LUMO & 0.1201 \\
\hline & & & & HOMO $\rightarrow$ LUMO & 0.6950 \\
\hline & & & & HOMO $\leftarrow$ LUMO & 0.1001 \\
\hline & $\mathrm{S} 0 \rightarrow \mathrm{S} 3$ & $2.9544 \mathrm{eV} 419.66 \mathrm{~nm}$ & 0.0075 & HOMO -2 $\rightarrow$ LUMO & 0.7028 \\
\hline \multirow[t]{8}{*}{ D4 } & $\mathrm{S} 0 \rightarrow \mathrm{S} 1$ & $2.1161 \mathrm{eV} 585.92 \mathrm{~nm}$ & 1.0618 & HOMO $\rightarrow$ LUMO & 0.7071 \\
\hline & $\mathrm{S} 0 \rightarrow \mathrm{S} 2$ & $2.4691 \mathrm{eV} 502.14 \mathrm{~nm}$ & 0.0017 & HOMO - $1 \rightarrow$ LUMO & 0.3592 \\
\hline & & & & $\mathrm{HOMO} \rightarrow \mathrm{LUMO}+1$ & 0.6078 \\
\hline & $\mathrm{S} 0 \rightarrow \mathrm{S} 3$ & $2.7612 \mathrm{eV} 449.02 \mathrm{~nm}$ & 0.0003 & HOMO -5 $\rightarrow$ LUMO & 0.1446 \\
\hline & & & & $\mathrm{HOMO}-3 \rightarrow \mathrm{LUMO}+1$ & 0.1484 \\
\hline & & & & HOMO $-2 \rightarrow$ LUMO +1 & 0.1227 \\
\hline & & & & HOMO -2 $\rightarrow$ LUMO & 0.6063 \\
\hline & & & & HOMO $-2 \rightarrow$ LUMO +1 & 0.2679 \\
\hline \multirow[t]{6}{*}{ FD2 } & $\mathrm{S} 0 \rightarrow \mathrm{S} 1$ & $2.2096 \mathrm{eV} 561.11 \mathrm{~nm}$ & 0.5639 & HOMO $-1 \rightarrow$ LUMO +1 & 0.1519 \\
\hline & & & & HOMO $\rightarrow$ LUMO & 0.6882 \\
\hline & $\mathrm{S} 0 \rightarrow \mathrm{S} 2$ & $2.3603 \mathrm{eV} 525.30 \mathrm{~nm}$ & 0.0012 & HOMO - $1 \rightarrow$ LUMO & 0.4289 \\
\hline & & & & $\mathrm{HOMO} \rightarrow \mathrm{LUMO}+1$ & 0.5590 \\
\hline & $\mathrm{S} 0 \rightarrow \mathrm{S} 5$ & $2.5225 \mathrm{eV} 491.51 \mathrm{~nm}$ & 1.0985 & HOMO $-1 \rightarrow$ LUMO + 1 & 0.6890 \\
\hline & & & & HOMO $\rightarrow$ LUMO & 0.1531 \\
\hline
\end{tabular}

[a] Only the selected low-lying excited states are presented. [b] Oscillator strength. [c] Only the main configurations are presented. [d] The CI coefficients are in absolute values. 
M2, optimized $\mathrm{S}_{0}$ state Geometry.

\begin{tabular}{|c|c|c|c|}
\hline B & 2.64879200 & 0.79462300 & 0.01606900 \\
\hline $\mathrm{C}$ & 2.53471600 & -1.83308800 & -0.00893200 \\
\hline $\mathrm{C}$ & 1.57984800 & -2.86723300 & -0.01243500 \\
\hline $\mathrm{C}$ & 0.34234800 & -2.23932800 & -0.00928500 \\
\hline $\mathrm{C}$ & 0.57222300 & -0.84162400 & -0.00466400 \\
\hline $\mathrm{C}$ & -0.34463400 & 0.22075300 & -0.00332200 \\
\hline $\mathrm{C}$ & 0.11746300 & 1.54169000 & -0.00578900 \\
\hline $\mathrm{C}$ & -0.59124900 & 2.77222100 & -0.01502000 \\
\hline $\mathrm{C}$ & 0.35427900 & 3.78944300 & -0.02212700 \\
\hline $\mathrm{C}$ & 1.61943900 & 3.16696200 & -0.01659600 \\
\hline $\mathrm{C}$ & -1.81378600 & -0.06354800 & -0.00067200 \\
\hline $\mathrm{C}$ & -2.49787800 & -0.19050400 & 1.22682800 \\
\hline $\mathrm{C}$ & -3.87235500 & -0.44895300 & 1.20356400 \\
\hline $\mathrm{C}$ & -4.58105100 & -0.58719800 & 0.00617600 \\
\hline $\mathrm{C}$ & -3.87770100 & -0.45205100 & -1.19469100 \\
\hline $\mathrm{C}$ & -2.50327600 & -0.19364400 & -1.22475500 \\
\hline $\mathrm{C}$ & -1.77991100 & -0.04730700 & 2.55048700 \\
\hline $\mathrm{C}$ & -6.05947300 & -0.89834200 & 0.01002800 \\
\hline $\mathrm{C}$ & -1.79125400 & -0.05368700 & -2.55197100 \\
\hline $\mathrm{F}$ & 3.39120500 & 0.94141300 & 1.17906200 \\
\hline $\mathrm{F}$ & 3.44532900 & 0.95397400 & -1.10808400 \\
\hline $\mathrm{N}$ & 1.95550700 & -0.61975900 & -0.00509300 \\
\hline $\mathrm{N}$ & 1.48315200 & 1.83042700 & -0.00663500 \\
\hline $\mathrm{H}$ & 1.79924800 & -3.92481800 & -0.01707800 \\
\hline $\mathrm{H}$ & -0.63160400 & -2.70858700 & -0.01115200 \\
\hline $\mathrm{H}$ & -1.66814700 & 2.86914200 & -0.01758500 \\
\hline $\mathrm{H}$ & 0.17656100 & 4.85581500 & -0.03102500 \\
\hline $\mathrm{H}$ & 2.60062500 & 3.62308600 & -0.01889200 \\
\hline $\mathrm{H}$ & -4.40163400 & -0.54315600 & 2.14941500 \\
\hline $\mathrm{H}$ & -4.41120300 & -0.54864200 & -2.13792400 \\
\hline $\mathrm{H}$ & -2.47327200 & -0.18892800 & 3.38414300 \\
\hline $\mathrm{H}$ & -1.32304600 & 0.94359900 & 2.65691700 \\
\hline $\mathrm{H}$ & -0.97361100 & -0.78267800 & 2.65454800 \\
\hline $\mathrm{H}$ & -6.55633300 & -0.49297000 & -0.87744700 \\
\hline $\mathrm{H}$ & -6.55276900 & -0.48760200 & 0.89703900 \\
\hline $\mathrm{H}$ & -6.23406300 & -1.98248300 & 0.01372000 \\
\hline $\mathrm{H}$ & -1.33395100 & 0.93657700 & -2.66236600 \\
\hline $\mathrm{H}$ & -2.48859800 & -0.19621800 & -3.38214100 \\
\hline $\mathrm{H}$ & -0.98617400 & -0.79005400 & -2.65837600 \\
\hline $\mathrm{Cl}$ & 4.24518200 & -2.04327900 & -0.00822300 \\
\hline
\end{tabular}

SCF done: -1489.98263031 Hartree

No imaginary Frequency. 
D4, optimized $\mathrm{S}_{0}$ state Geometry.

$\mathrm{S}$

$\mathrm{F}$

F

$\mathrm{F}$

F

$\mathrm{N}$

$\mathrm{N}$

$\mathrm{N}$

$\mathrm{N}$

C

C

$\mathrm{H}$

C

C

C

C

C

C

$\mathrm{H}$

C

C

$\mathrm{H}$

C

$\mathrm{H}$

$\mathrm{C}$

$\mathrm{H}$

C

C

$\mathrm{H}$

C

C

C

C

C

C

C

C

C

$\mathrm{H}$

$\mathrm{H}$

$\mathrm{H}$

C

\begin{tabular}{|c|c|c|}
\hline-0.00010700 & -1.36508000 & -0.00110500 \\
\hline-2.55002700 & -2.95104700 & -0.43125800 \\
\hline 2.52529700 & -1.66002000 & 2.32558100 \\
\hline 2.54916800 & -2.95107700 & 0.43178900 \\
\hline-2.52661000 & -1.66176000 & -2.32624400 \\
\hline-2.58663100 & -0.55607900 & -0.16961100 \\
\hline 2.58649000 & -0.55637000 & 0.16792500 \\
\hline-4.58520200 & -1.76824900 & -1.05672800 \\
\hline 4.58437000 & -1.76823200 & 1.05698200 \\
\hline 3.43794100 & 0.46395300 & -0.26049300 \\
\hline 1.34822700 & 0.99470100 & -0.93751700 \\
\hline 0.48792100 & 1.46765500 & -1.38758700 \\
\hline 1.33746900 & -0.24019200 & -0.24071500 \\
\hline-5.37985100 & -0.71020600 & -0.59850900 \\
\hline-3.43769000 & 0.46480100 & 0.25829200 \\
\hline 4.81936400 & 0.40345900 & -0.04443200 \\
\hline-4.81924100 & 0.40430300 & 0.04315700 \\
\hline 2.65941900 & 1.43614400 & -0.93988600 \\
\hline 3.04811700 & 2.33694600 & -1.39428700 \\
\hline 5.37942600 & -0.71062600 & 0.59842800 \\
\hline-6.73436900 & -1.01135500 & -0.88123600 \\
\hline-7.57590500 & -0.37908900 & -0.63459900 \\
\hline-2.65862000 & 1.43752900 & 0.93631400 \\
\hline-3.04690000 & 2.33889800 & 1.38995400 \\
\hline 6.73377300 & -1.01182500 & 0.88206800 \\
\hline 7.57554400 & -0.37991900 & 0.63530700 \\
\hline-5.70137100 & 1.51873700 & 0.51084500 \\
\hline-1.34752200 & 0.99586000 & 0.93368000 \\
\hline-0.48688400 & 1.46911600 & 1.38278800 \\
\hline-1.33735200 & -0.23973200 & 0.23806700 \\
\hline 5.42469400 & -2.66911400 & 1.59949900 \\
\hline 5.70191300 & 1.51734200 & -0.51262500 \\
\hline-5.42596700 & -2.66938600 & -1.59818700 \\
\hline 6.76446400 & -2.24864800 & 1.51138600 \\
\hline-6.76560200 & -2.24862600 & -1.50968600 \\
\hline 6.26467000 & 1.46915600 & -1.80416400 \\
\hline-5.96127500 & 2.60868300 & -0.34599100 \\
\hline-5.36567100 & 2.68301500 & -1.73458500 \\
\hline-5.71197800 & 3.57931900 & -2.25663900 \\
\hline-5.64193200 & 1.81229200 & -2.34038800 \\
\hline-4.27006400 & 2.71468600 & -1.70341300 \\
\hline 5.96337000 & 2.60827300 & 0.34577400 \\
\hline
\end{tabular}




\begin{tabular}{|c|c|c|c|}
\hline $\mathrm{C}$ & 5.99717900 & 0.31367600 & -2.74321000 \\
\hline $\mathrm{H}$ & 6.32387600 & -0.64068600 & -2.31421500 \\
\hline $\mathrm{H}$ & 6.52576800 & 0.45465000 & -3.69005900 \\
\hline $\mathrm{H}$ & 4.92852100 & 0.21349500 & -2.96654500 \\
\hline $\mathrm{C}$ & -6.26201700 & 1.47242200 & 1.80554600 \\
\hline $\mathrm{C}$ & -6.78661000 & 3.64051600 & 0.11392800 \\
\hline $\mathrm{H}$ & -6.98592800 & 4.48282000 & -0.54506800 \\
\hline $\mathrm{C}$ & 7.08712300 & 2.52505300 & -2.21607100 \\
\hline $\mathrm{H}$ & 7.52167800 & 2.48953300 & -3.21261500 \\
\hline B & 3.01428400 & -1.79073900 & 1.03464500 \\
\hline B & -3.01513300 & -1.79115200 & -1.03499700 \\
\hline $\mathrm{C}$ & -7.08000700 & 2.52875600 & 2.21867100 \\
\hline $\mathrm{H}$ & -7.50973700 & 2.49624200 & 3.21765000 \\
\hline $\mathrm{C}$ & 5.36770800 & 2.68057700 & 1.73444700 \\
\hline $\mathrm{H}$ & 4.27202900 & 2.70893700 & 1.70318600 \\
\hline $\mathrm{H}$ & 5.71136900 & 3.57783300 & 2.25660600 \\
\hline $\mathrm{H}$ & 5.64650000 & 1.81052400 & 2.34009300 \\
\hline $\mathrm{C}$ & -7.35834100 & 3.62005000 & 1.38931500 \\
\hline $\mathrm{C}$ & -5.99097500 & 0.31766200 & 2.74444700 \\
\hline $\mathrm{H}$ & -6.51717000 & 0.45864700 & 3.69262400 \\
\hline $\mathrm{H}$ & -4.92163500 & 0.21824700 & 2.96485000 \\
\hline $\mathrm{H}$ & -6.31818300 & -0.63703700 & 2.31658100 \\
\hline $\mathrm{C}$ & 7.36369300 & 3.61588900 & -1.38884300 \\
\hline $\mathrm{C}$ & 6.79087600 & 3.63681300 & -0.11176700 \\
\hline $\mathrm{H}$ & 6.99347200 & 4.47721700 & 0.54907100 \\
\hline $\mathrm{C}$ & -8.26999100 & 4.73258600 & 1.85150100 \\
\hline $\mathrm{H}$ & -8.07200100 & 5.66298200 & 1.30978400 \\
\hline $\mathrm{H}$ & -8.15220800 & 4.92746400 & 2.92275600 \\
\hline $\mathrm{H}$ & -9.32398500 & 4.47429200 & 1.68379000 \\
\hline $\mathrm{C}$ & 8.25635300 & 4.74377500 & -1.85111000 \\
\hline $\mathrm{H}$ & 9.14007900 & 4.83975000 & -1.20837800 \\
\hline $\mathrm{H}$ & 7.73107500 & 5.70600200 & -1.81744500 \\
\hline $\mathrm{H}$ & 8.60263700 & 4.58473400 & -2.87673300 \\
\hline $\mathrm{H}$ & -7.62207300 & -2.80213200 & -1.86580200 \\
\hline $\mathrm{H}$ & 7.62064100 & -2.80209500 & 1.86829200 \\
\hline $\mathrm{Cl}$ & 4.87715000 & -4.14069400 & 2.30799600 \\
\hline $\mathrm{Cl}$ & -4.87900200 & -4.14148700 & -2.30605100 \\
\hline
\end{tabular}

SCF done: -3376.95219378 Hartree

No imaginary Frequency.

FD2, optimized $\mathrm{S}_{0}$ state Geometry.
$\mathrm{S}$
$0.00145400 \quad-2.41723900$
0.00127400
F
$-3.07216400 \quad-3.37969200$
0.71947800 


\begin{tabular}{|c|c|c|}
\hline 3.35546600 & -2.94402100 & 1.5100 \\
\hline 3.07475300 & -3.37769800 & -0.71749100 \\
\hline 35024100 & -2.94679700 & -1.50930600 \\
\hline 2.54730900 & -1.17714900 & -0.07183900 \\
\hline 2.54937600 & -1.17533700 & 0.07384100 \\
\hline-4.91643700 & -1.92392000 & 0.02962400 \\
\hline 4.91887600 & -1.92039700 & -0.03045400 \\
\hline 2.97931200 & 0.16460300 & 0.06965500 \\
\hline 0.72087400 & 0.19811300 & 0.02212300 \\
\hline 1.21746900 & -1.14028800 & 0.03715100 \\
\hline-5.29089800 & & 0.03842800 \\
\hline-2.97822300 & 0.16247700 & -0.06767900 \\
\hline 4.34375800 & 0.46617000 & .02200600 \\
\hline-4.34292700 & 0.46307100 & -0.02188800 \\
\hline 1.84233400 & 1.01912900 & 0.04459200 \\
\hline 1.88026700 & 2.09919100 & 0.02833800 \\
\hline 5.29236000 & -0.56925300 & -0.03999700 \\
\hline-6.70035600 & -0.50164200 & .14738300 \\
\hline-7.27443500 & 0.41354500 & 0.18769100 \\
\hline-1.84186800 & 1.01781800 & -0.04147900 \\
\hline-1.88062700 & 2.09785200 & -0.02500000 \\
\hline 6.70156500 & -0.49685900 & 77400 \\
\hline 7.27494500 & 41871600 & -0.19344500 \\
\hline-4.79617700 & 1.88912200 & 1087400 \\
\hline-0.71983000 & 0.19761800 & -0.01872900 \\
\hline-1.21546200 & -1.14115100 & -0.03437600 \\
\hline 6.05821800 & -2.63194900 & -0.12596400 \\
\hline 4.79520600 & 1.89279200 & 0.01056300 \\
\hline-6.05547200 & -2.63627600 & 9100 \\
\hline 7.18632400 & -1.79703700 & -0.20944300 \\
\hline-7.18431500 & -1.80216300 & 0.20426600 \\
\hline 5.04784100 & 2.55232600 & 1.23282600 \\
\hline-5.03727600 & 2.55195300 & -1.23326200 \\
\hline-4.84745800 & 1.85338900 & -2.56140200 \\
\hline-5.09053100 & 2.52449700 & -3.38987700 \\
\hline-5.48783300 & 0.96761800 & -2.64674800 \\
\hline-3.81392900 & 1.51344000 & -2.69572800 \\
\hline 4.96939000 & 2.56386000 & -1.21794400 \\
\hline 4.87289600 & 1.84892700 & 2.56043800 \\
\hline 3.84257200 & 1.50192000 & 2.70113700 \\
\hline 5.11740700 & 2.51955300 & 3.38888400 \\
\hline 5.51953400 & 0.96708800 & 2.63905300 \\
\hline-4.97543900 & 2.55901200 & 1.21797000 \\
\hline \multirow[t]{2}{*}{-5.45953700} & 3.88466700 & -1.20011800 \\
\hline & \multicolumn{2}{|r|}{530} \\
\hline
\end{tabular}




\begin{tabular}{|c|c|c|c|}
\hline $\mathrm{H}$ & -5.64329900 & 4.39755100 & -2.14182600 \\
\hline $\mathrm{C}$ & 5.47346400 & 3.88355500 & 1.19936800 \\
\hline $\mathrm{H}$ & 5.67317100 & 4.39163400 & 2.14049900 \\
\hline B & 3.46149700 & -2.43813600 & 0.22377200 \\
\hline B & -3.45836100 & -2.44065400 & -0.22252200 \\
\hline $\mathrm{C}$ & -5.39853900 & 3.89223700 & 1.19763900 \\
\hline $\mathrm{H}$ & -5.53303400 & 4.41140700 & 2.14414600 \\
\hline $\mathrm{C}$ & 4.70984700 & 1.87640400 & -2.54027700 \\
\hline $\mathrm{H}$ & 5.35776000 & 1.00261100 & -2.67581900 \\
\hline $\mathrm{H}$ & 4.89093500 & 2.56056700 & -3.37390500 \\
\hline $\mathrm{H}$ & 3.67548800 & 1.52117600 & -2.61536500 \\
\hline $\mathrm{C}$ & -5.65033500 & 4.57260900 & 0.00244500 \\
\hline $\mathrm{C}$ & -4.71723600 & 1.87112000 & 2.54033600 \\
\hline $\mathrm{H}$ & -4.89973000 & 2.55485800 & 3.37400700 \\
\hline $\mathrm{H}$ & -3.68299300 & 1.51584600 & 2.61679400 \\
\hline $\mathrm{H}$ & -5.36535400 & 0.99727200 & 2.67449900 \\
\hline $\mathrm{C}$ & 5.65070200 & 4.57576000 & -0.00325300 \\
\hline $\mathrm{C}$ & 5.39593200 & 3.89639300 & -1.19792000 \\
\hline $\mathrm{H}$ & 5.53291400 & 4.41466400 & -2.14449800 \\
\hline $\mathrm{C}$ & -6.13763800 & 6.00254900 & 0.00979000 \\
\hline $\mathrm{H}$ & -5.78402400 & 6.55060400 & -0.86977400 \\
\hline $\mathrm{H}$ & -5.80071700 & 6.53564100 & 0.90469800 \\
\hline $\mathrm{H}$ & -7.23486000 & 6.04494600 & -0.00106100 \\
\hline $\mathrm{C}$ & 6.08262500 & 6.02341800 & -0.00685200 \\
\hline $\mathrm{H}$ & 5.22486600 & 6.69115500 & 0.14838000 \\
\hline $\mathrm{H}$ & 6.54452800 & 6.30100200 & -0.95956600 \\
\hline $\mathrm{H}$ & 6.79986700 & 6.22888700 & 0.79496900 \\
\hline $\mathrm{H}$ & -8.20716900 & -2.13679100 & 0.29632800 \\
\hline $\mathrm{H}$ & 8.20922800 & -2.13095800 & -0.30348300 \\
\hline $\mathrm{Cl}$ & 6.07553500 & -4.35461600 & -0.13712200 \\
\hline $\mathrm{Cl}$ & -6.07156800 & -4.35895900 & 0.13417000 \\
\hline
\end{tabular}

SCF done: -3375.75277002 Hartree

No imaginary Frequency. 


\section{Photothermal studies}

Preparation of T4 nanoparticles (T4 NPs). T4 NPs were fabricated following literature procedures. ${ }^{10}$ Tetramer T4 (374 $\mu \mathrm{L}, 267.6 \mu \mathrm{M}$ in chloroform) and F127 (680 $\mu \mathrm{L}, 7.7 \mathrm{mg} \mathrm{mL}^{-1}$ in chloroform) were added into a flask with $5 \mathrm{~mL}$ chloroform. And the mixture sonicated for $20 \mathrm{~min}$ at room temperature. The chloroform was evaporated entirely under reduced pressure, after that, water $(5 \mathrm{~mL})$ was added and shaken. Finally, the liquid was stored at $4{ }^{\circ} \mathrm{C}$ for further experiments.

Photothermal experiments. To demonstrate the photothermal conversion behavior of T4, the solution containing T4 in DMSO or T4 NPs in water at the concentration of $20.0 \mu \mathrm{g} \mathrm{mL}^{-1}$ was used. The solution of T4 or T4 NPs was irradiated under $808 \mathrm{~nm}$ laser $\left(0.53 \mathrm{~W} \mathrm{~cm}^{-2}\right)$ for 600 or $900 \mathrm{~s}$, and then cooled down to room temperature. Indocyanine green (ICG) in DMSO or water as the reference was tested at a concentration of $80 \mu \mathrm{g} \mathrm{mL}^{-1}$ under $808 \mathrm{~nm}$ irradiation $\left(0.53 \mathrm{~W} \mathrm{~cm}^{-2}\right)$. The photothermal conversion efficiency $(\eta)$ values were calculated by a reported method. ${ }^{11}$

Photothermal activity and photothermal conversion efficiency. The sample was irradiated with $808 \mathrm{~nm}$ laser $\left(0.53 \mathrm{~W} \mathrm{~cm}^{-2}\right)$ with pure solution as the blank control group. The used amount of sample was $0.2 \mathrm{~mL}$. We recorded the temperature at $20 \mathrm{~s}$ intervals, and calculated photothermal conversion efficiency according to the reference method. ${ }^{11}$ Detailed calculation was given as following:

$$
\eta=\frac{h \mathrm{~A} \Delta \mathrm{T}_{\max }-\mathrm{Q}_{\mathrm{s}}}{\mathrm{I}\left(1-10^{-\mathrm{A}_{\lambda}}\right)}
$$

where $h$ is the heat transfer coefficient; A is the surface area of the container, and $h \mathrm{~A}$ can be determined by eq $\mathrm{S} 2 . \Delta \mathrm{T}_{\max }$ is the temperature change of the sample dispersion at the maximum steady state temperature; $I$ is the laser power; $A_{\lambda}$ is the absorbance of sample at the wavelength of $808 \mathrm{~nm}$, and $\eta$ is the conversion efficiency from the absorbed light energy to thermal energy. $\mathrm{Q}_{\mathrm{s}}$ is heat associated with the light absorbed by solvent per second and can be determined by eq S5. 


$$
h A=\frac{\Sigma m_{i} C_{p i}}{\tau_{s}}
$$

where $m$ and $C_{p}$ are the mass and heat capacity of solvent, $\tau_{\mathrm{s}}$ is the time constant can be determined according to the eq S3 and eq S4.

$$
\begin{gathered}
\tau_{s}=-\ln \theta \\
\theta=\frac{\Delta T}{\Delta T_{\max }}
\end{gathered}
$$

Where $\Delta \mathrm{T}_{\max }$ is the temperature change of the sample dispersion at the maximum steady state temperature; $\Delta \mathrm{T}$ is defined as $\mathrm{T}$-Tsurr ( $\mathrm{T}$ and Tsurr are the solution temperature and ambient temperature of the surroundings, respectively).

$$
Q_{s}=\frac{m C_{p} \Delta T_{\max }}{t}
$$

$\mathrm{Q}_{s}$ is the heat associated with the light absorbance of the solvent, which is measured independently using pure solution without sample; $\Delta \mathrm{T}_{\max }$ is the temperature change of pure solution; $t$ is the irradiation time of the laser. 

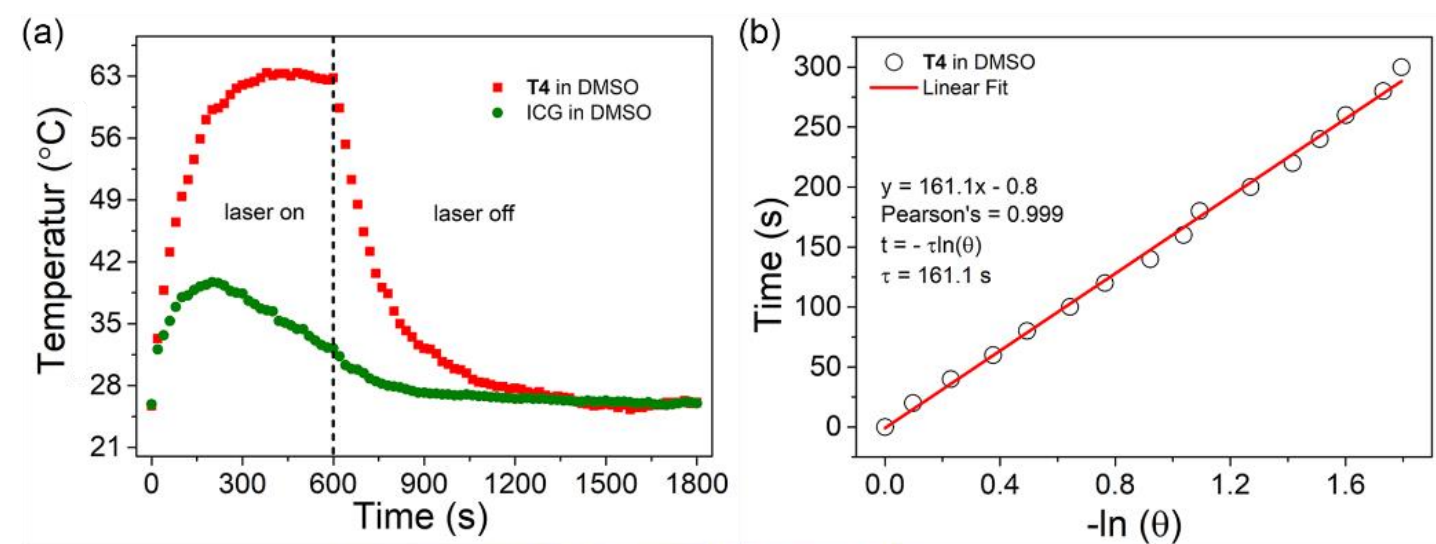

(c)

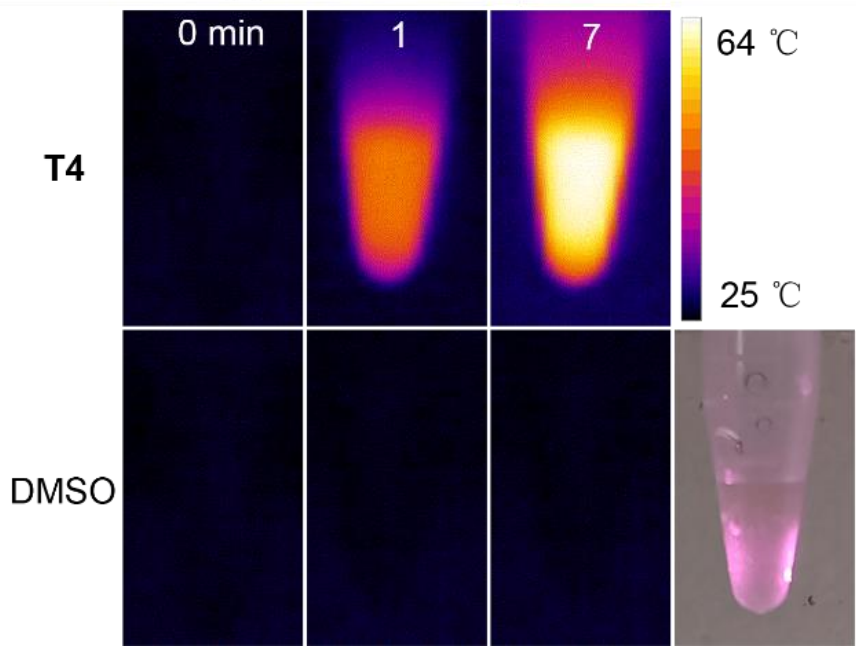

Figure S23. (a) Photothermal heating curves of $20 \mu \mathrm{M}$ T4 and $80 \mu \mathrm{M}$ ICG under irradiation $\left(0.53 \mathrm{~W} \mathrm{~cm}^{-2}\right)$ in DMSO; (b) Plot of cooling time versus negative natural logarithm of the temperature obtained from the cooling stage of (a); (c) Infrared thermal images of $20 \mu \mathrm{M}$ T4 or pure DMSO recorded under $808 \mathrm{~nm}$ laser exposure $(0.53 \mathrm{~W}$ $\left.\mathrm{cm}^{-2}, 7 \mathrm{~min}\right)$. 
8. Copies of ${ }^{1} \mathrm{H}$ and ${ }^{13} \mathrm{C}$ NMR spectra
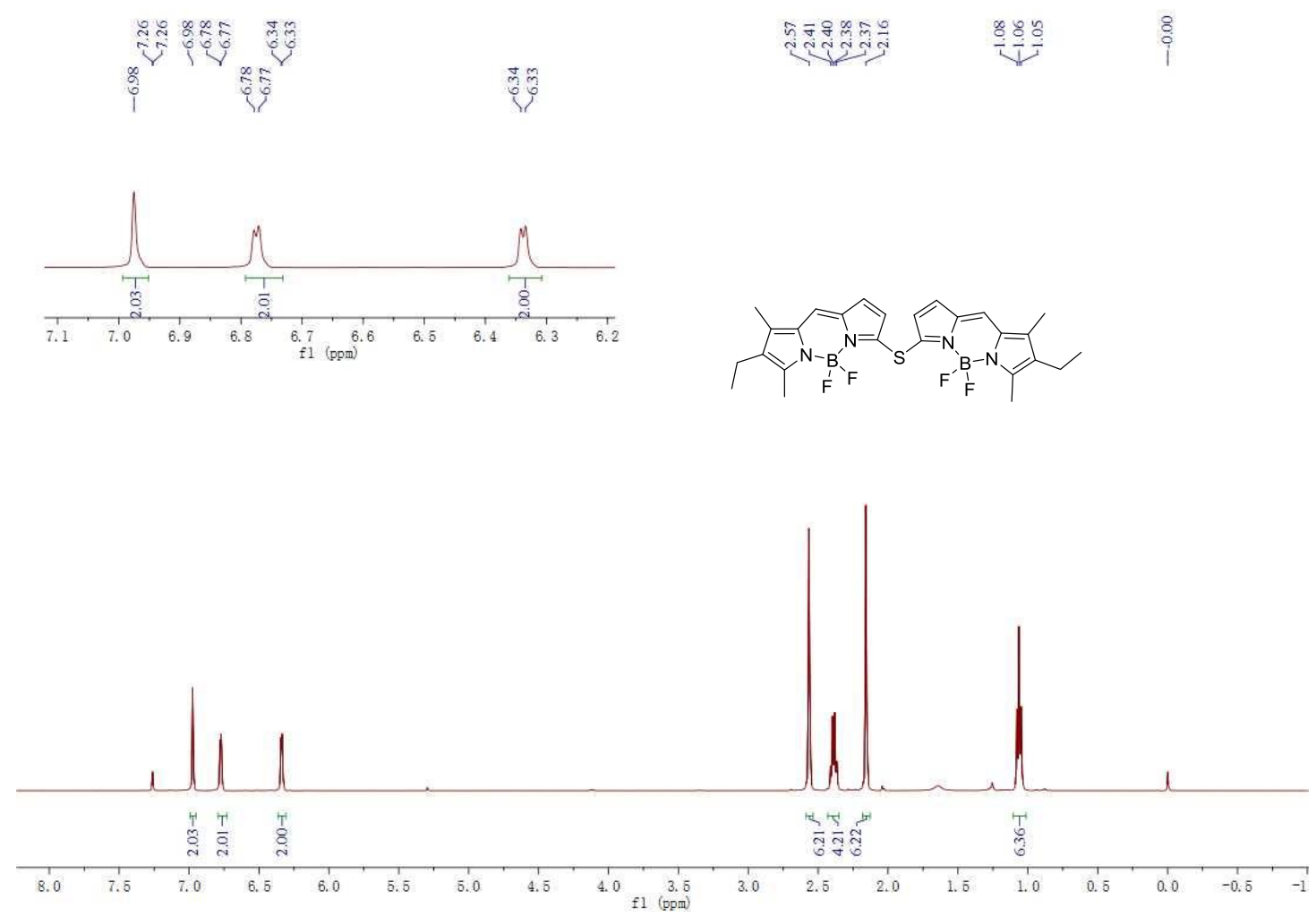

${ }^{1} \mathrm{H}$ NMR spectrum of $\mathbf{D 1}\left(\mathrm{CDCl}_{3}, 500 \mathrm{MHz}\right)$
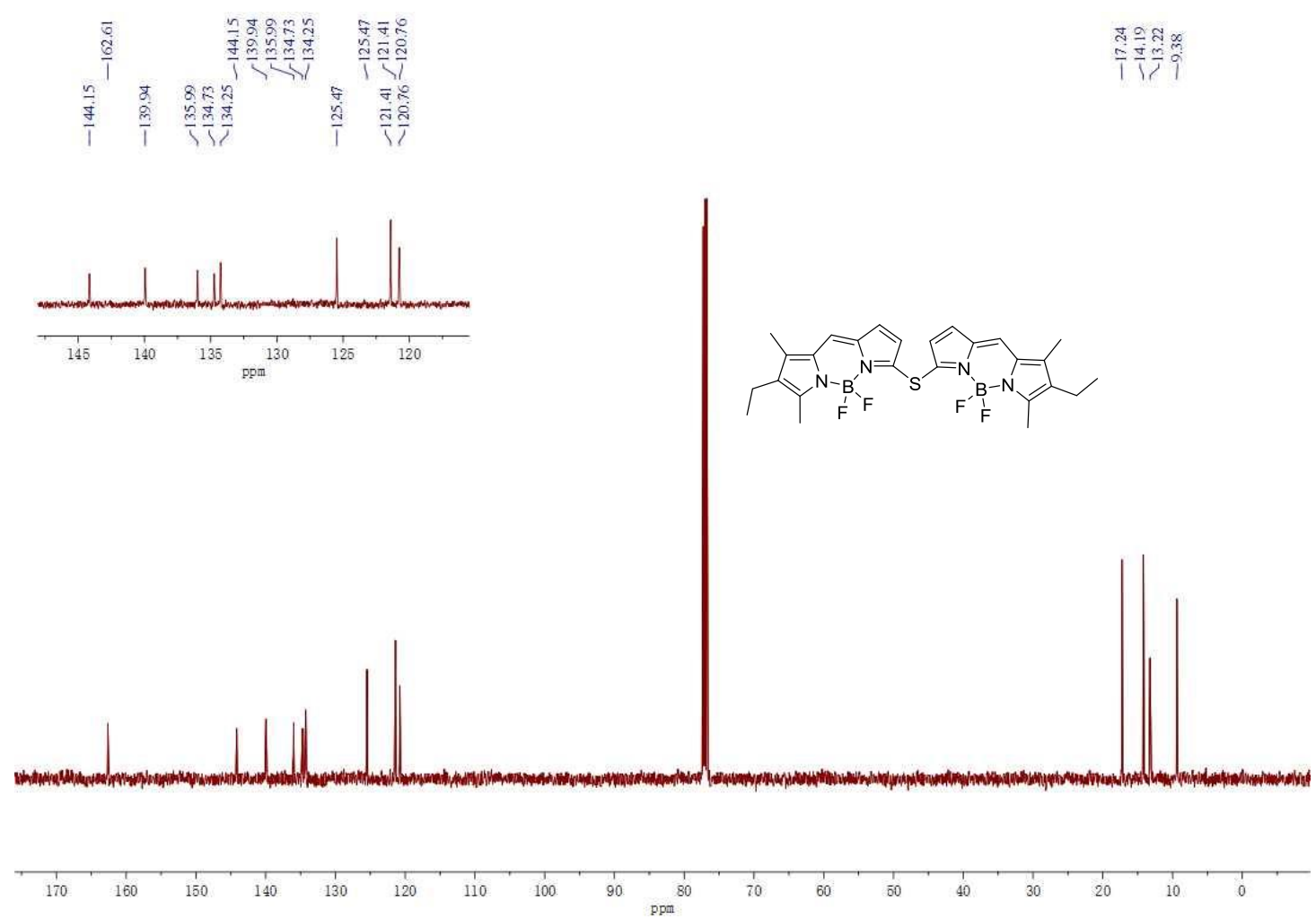

${ }^{13} \mathrm{C}$ NMR spectrum of $\mathbf{D 1}\left(\mathrm{CDCl}_{3}, 126 \mathrm{MHz}\right)$ 


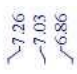

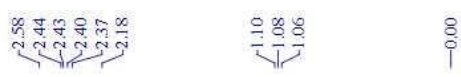

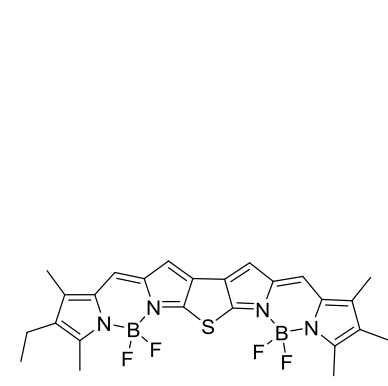

$\mid$
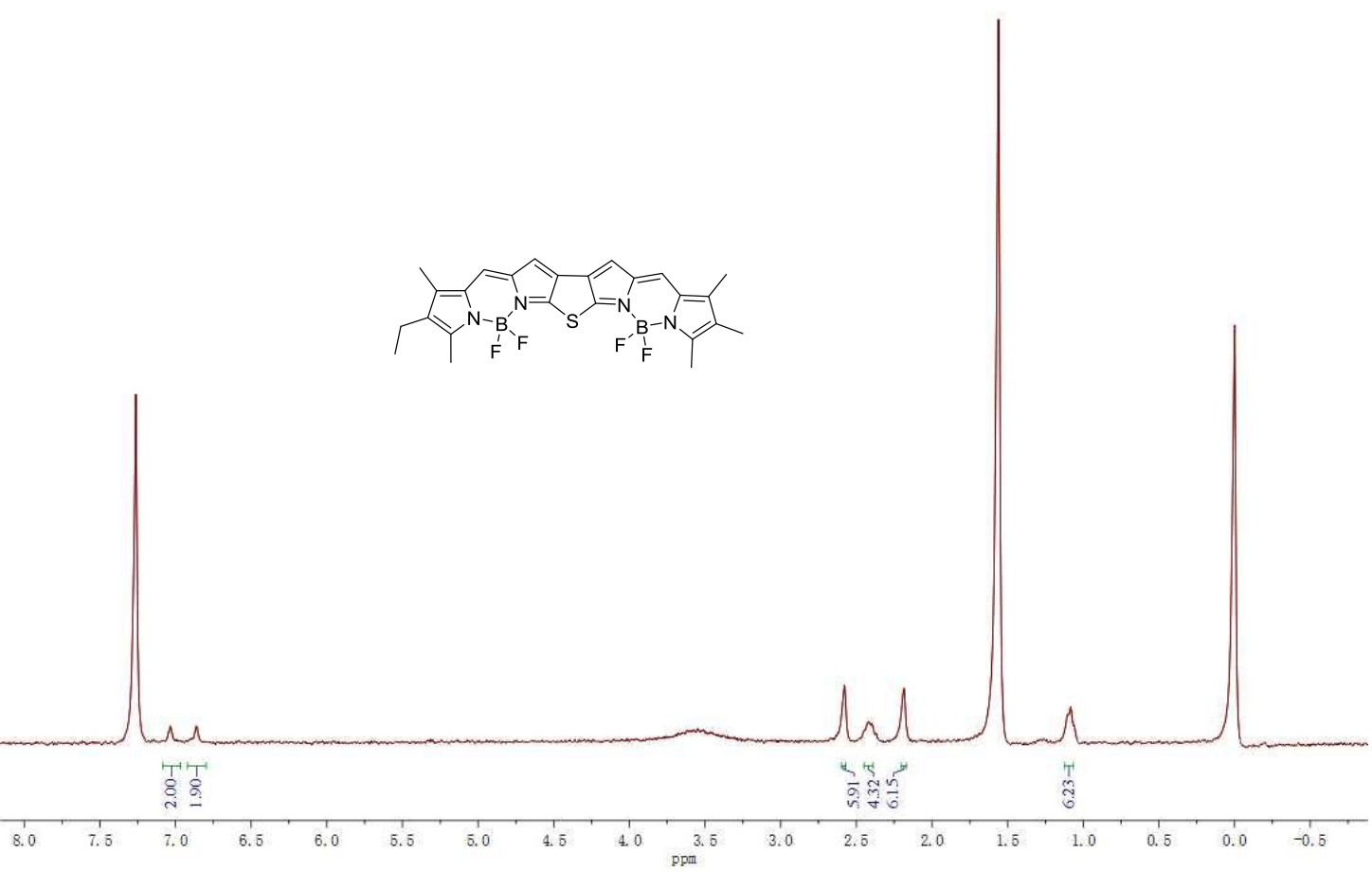

${ }^{1} \mathrm{H}$ NMR spectrum of FD1 $\left(\mathrm{CDCl}_{3}, 300 \mathrm{MHz}\right)$ 


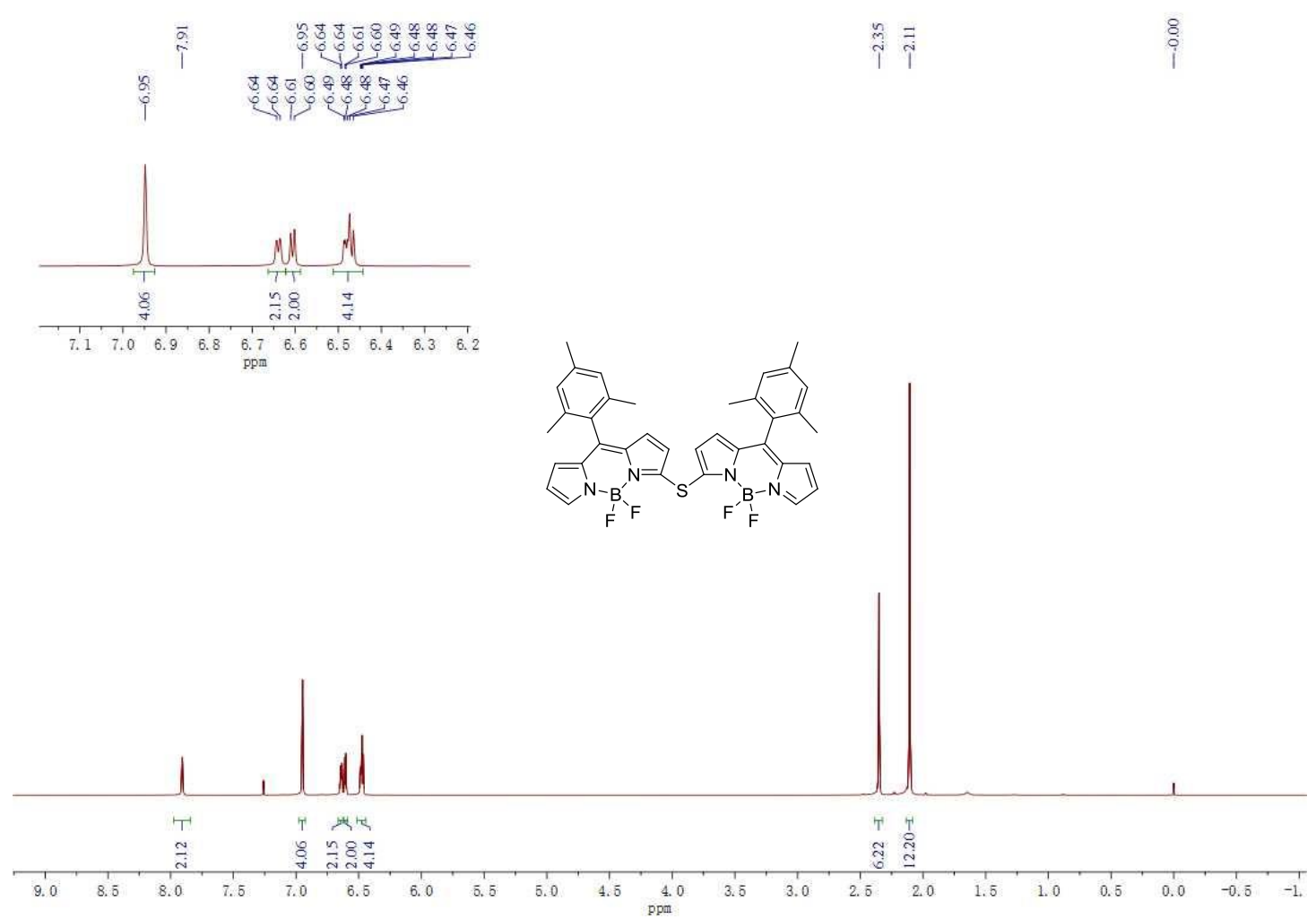

${ }^{1} \mathrm{H}$ NMR spectrum of $\mathbf{D 2}\left(\mathrm{CDCl}_{3}, 500 \mathrm{MHz}\right)$
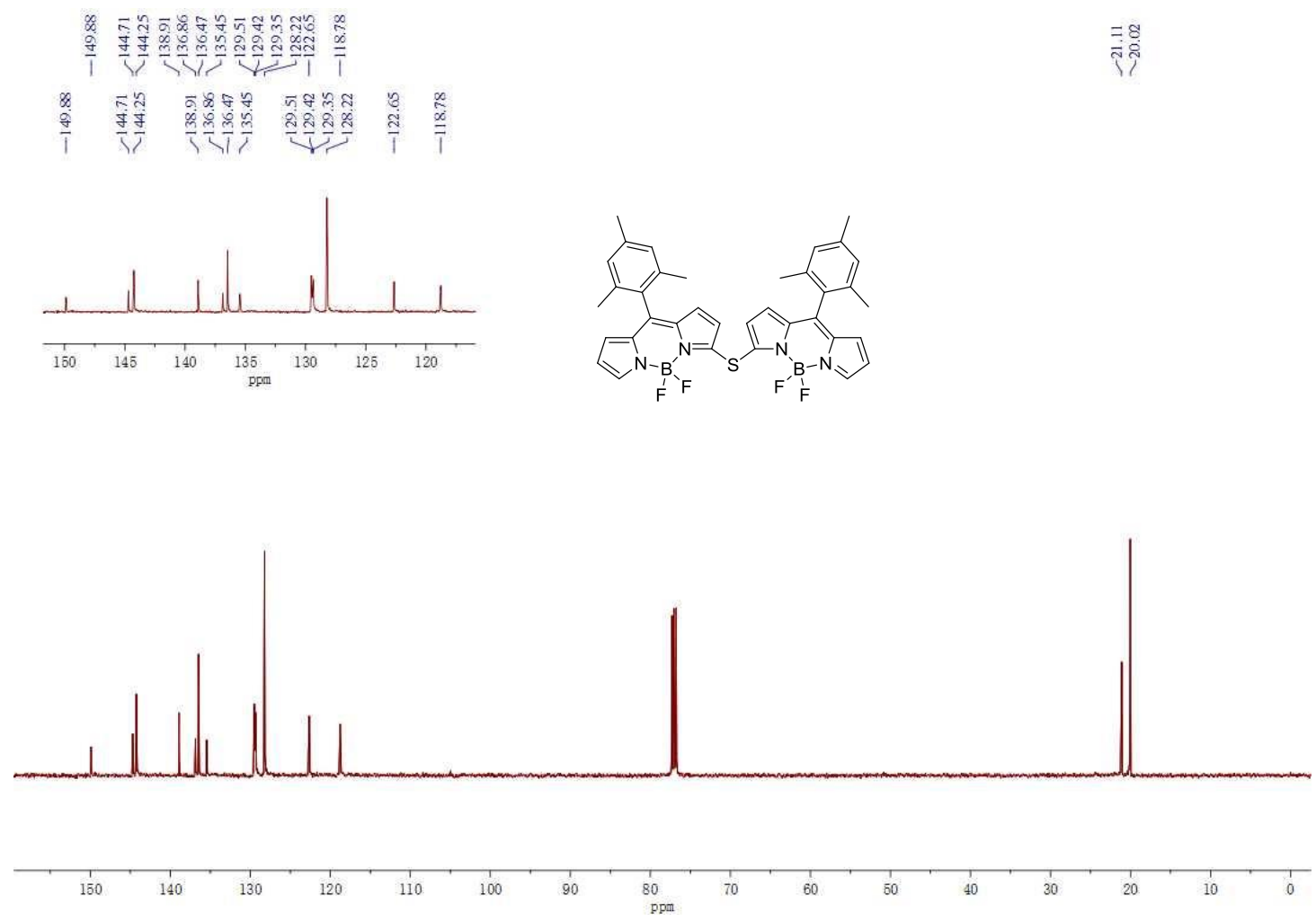

${ }^{13} \mathrm{C}$ NMR spectrum of $\mathbf{D 2}\left(\mathrm{CDCl}_{3}, 126 \mathrm{MHz}\right)$ 


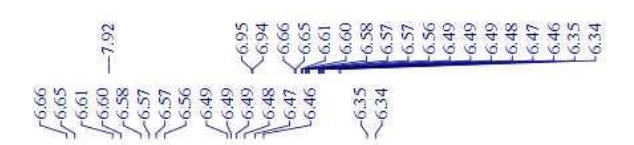

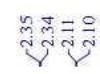
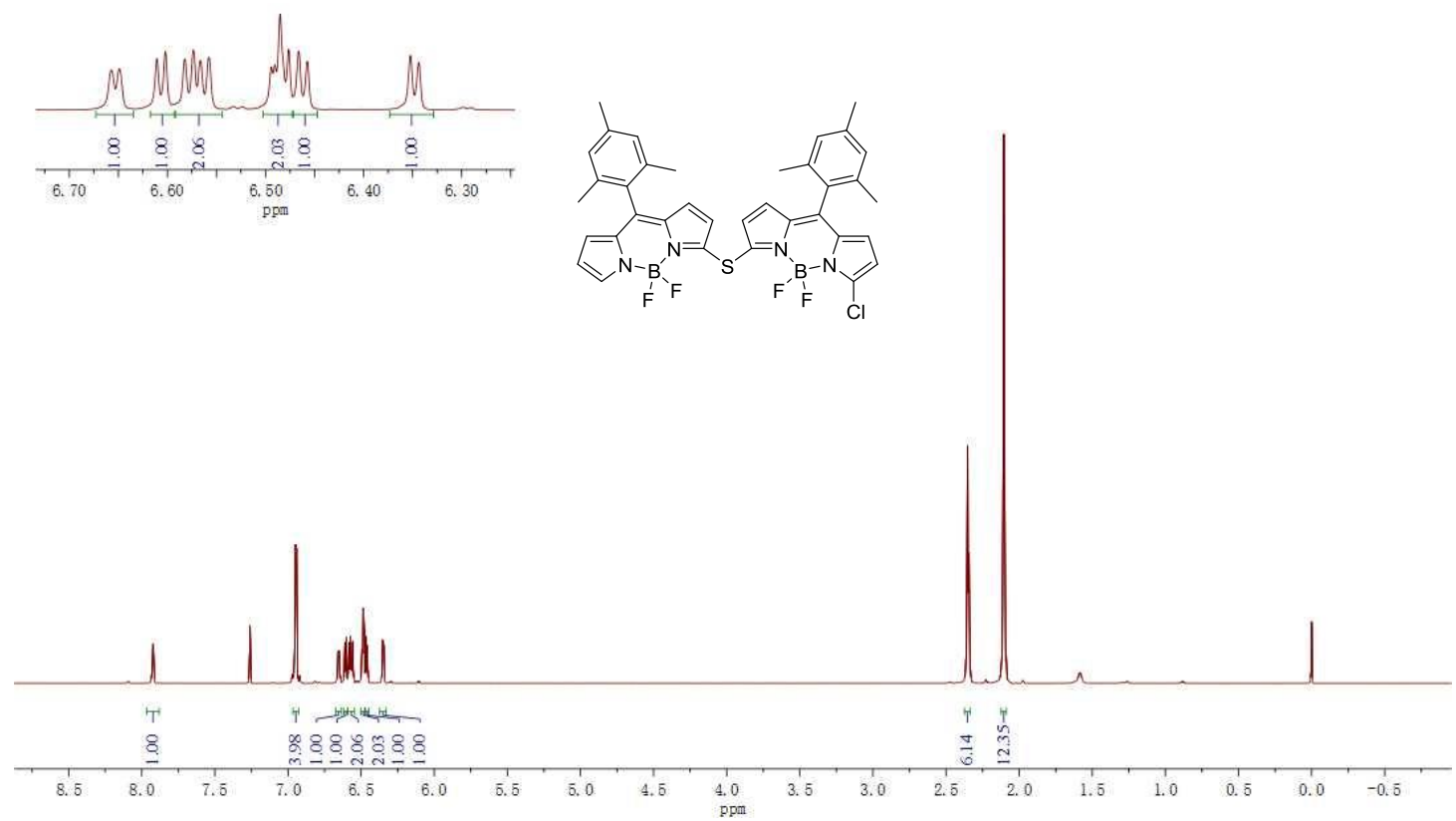

${ }^{1} \mathrm{H}$ NMR spectrum of $\mathbf{D 3}\left(\mathrm{CDCl}_{3}, 500 \mathrm{MHz}\right)$
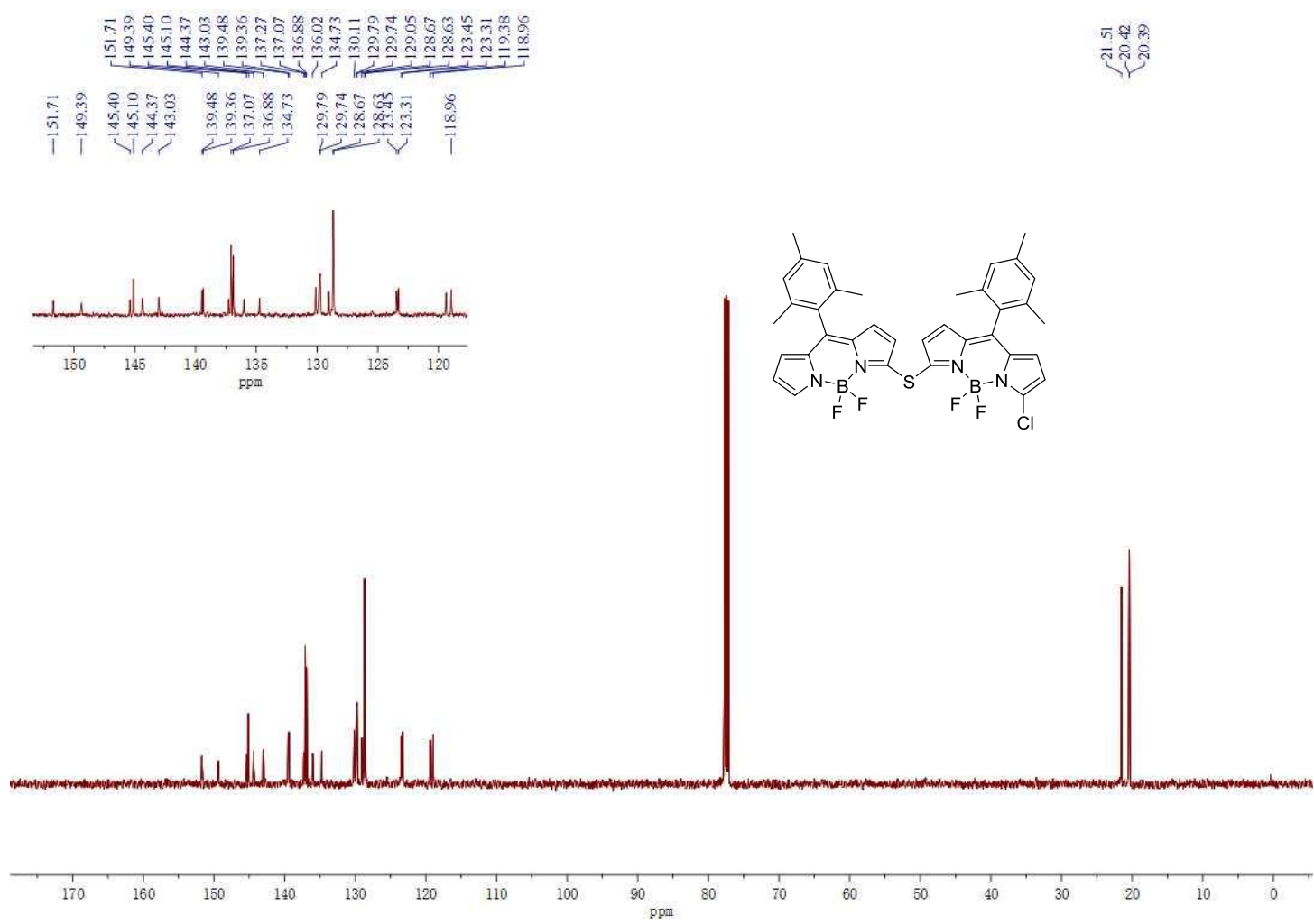

${ }^{13} \mathrm{C}$ NMR spectrum of $\mathbf{D 3}\left(\mathrm{CDCl}_{3}, 126 \mathrm{MHz}\right)$ 


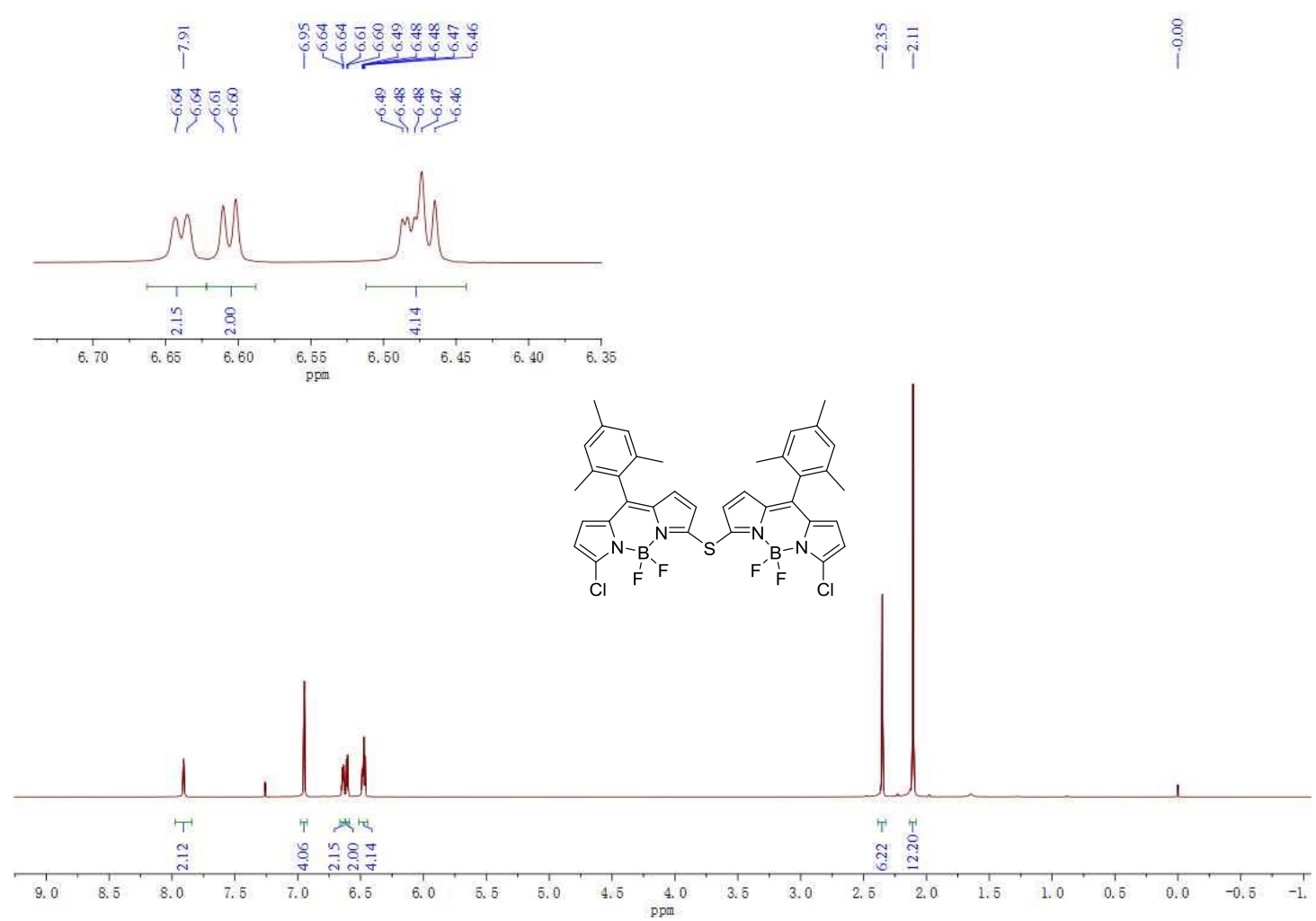

${ }^{1} \mathrm{H}$ NMR spectrum of $\mathbf{D 4}\left(\mathrm{CDCl}_{3}, 500 \mathrm{MHz}\right)$
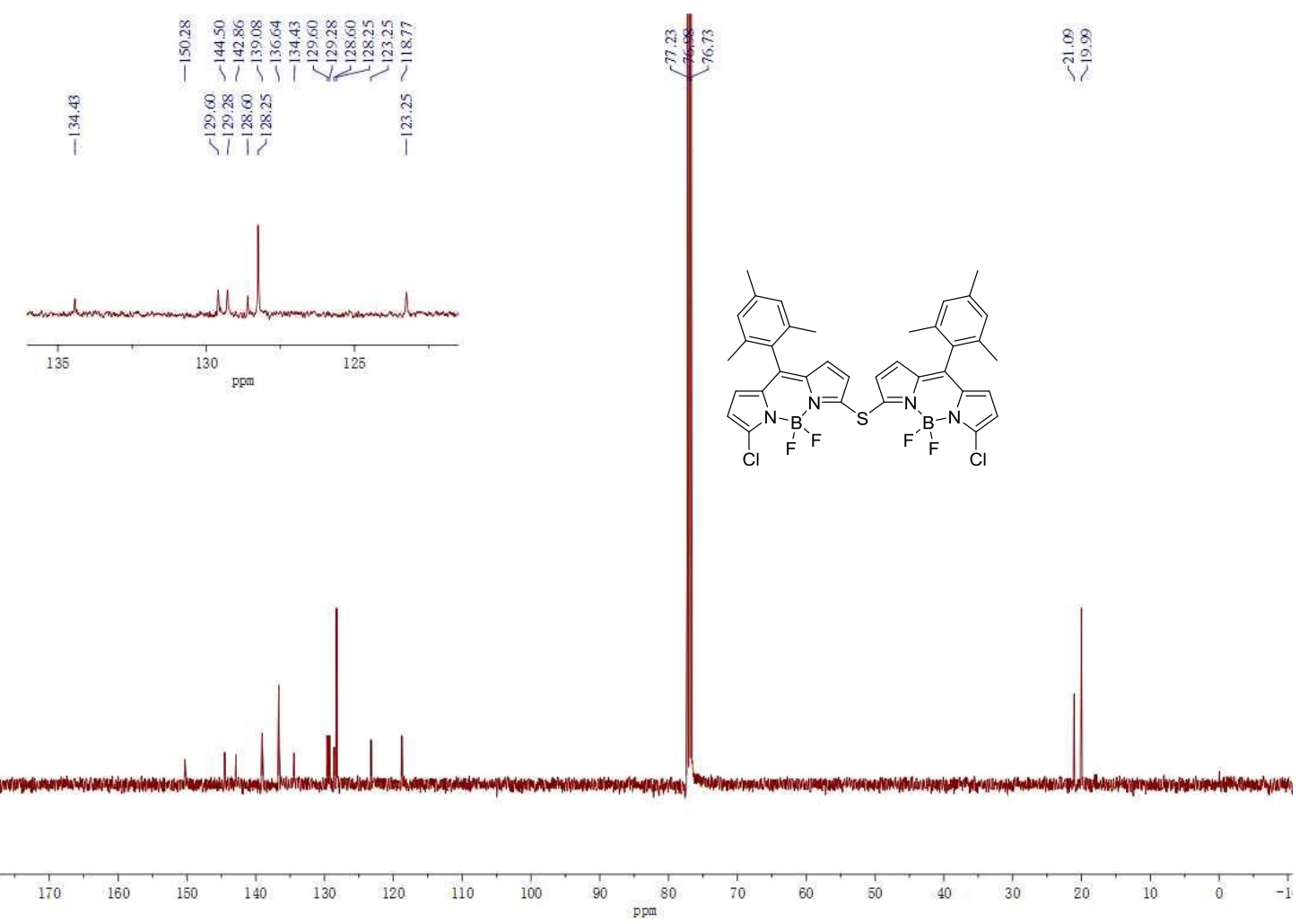

${ }^{13} \mathrm{C}$ NMR spectrum of $\mathbf{D 4}\left(\mathrm{CDCl}_{3}, 126 \mathrm{MHz}\right)$ 

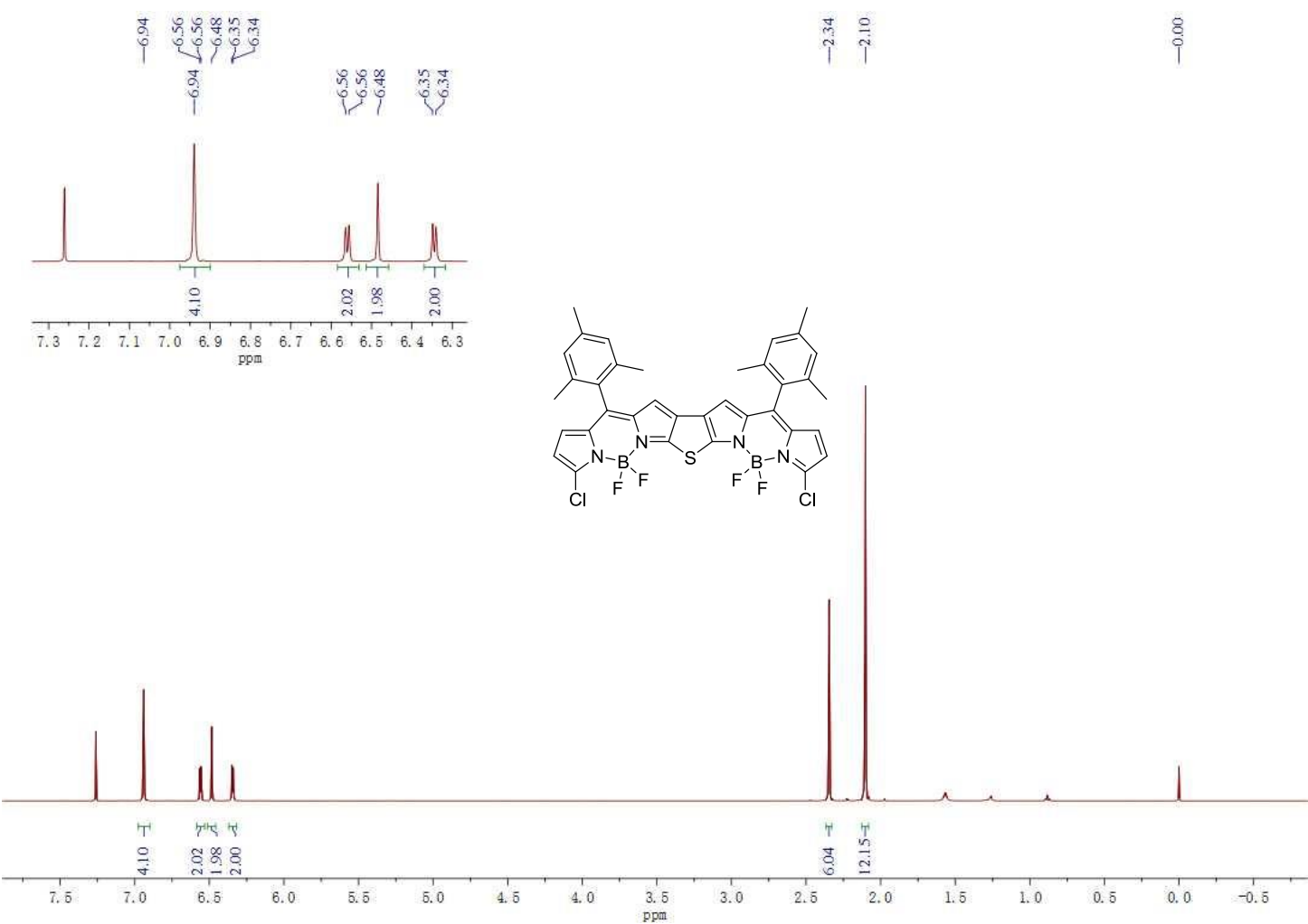

${ }^{1} \mathrm{H}$ NMR spectrum of FD2 $\left(\mathrm{CDCl}_{3}, 500 \mathrm{MHz}\right)$

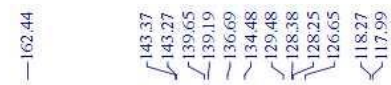

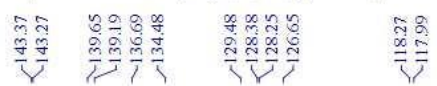
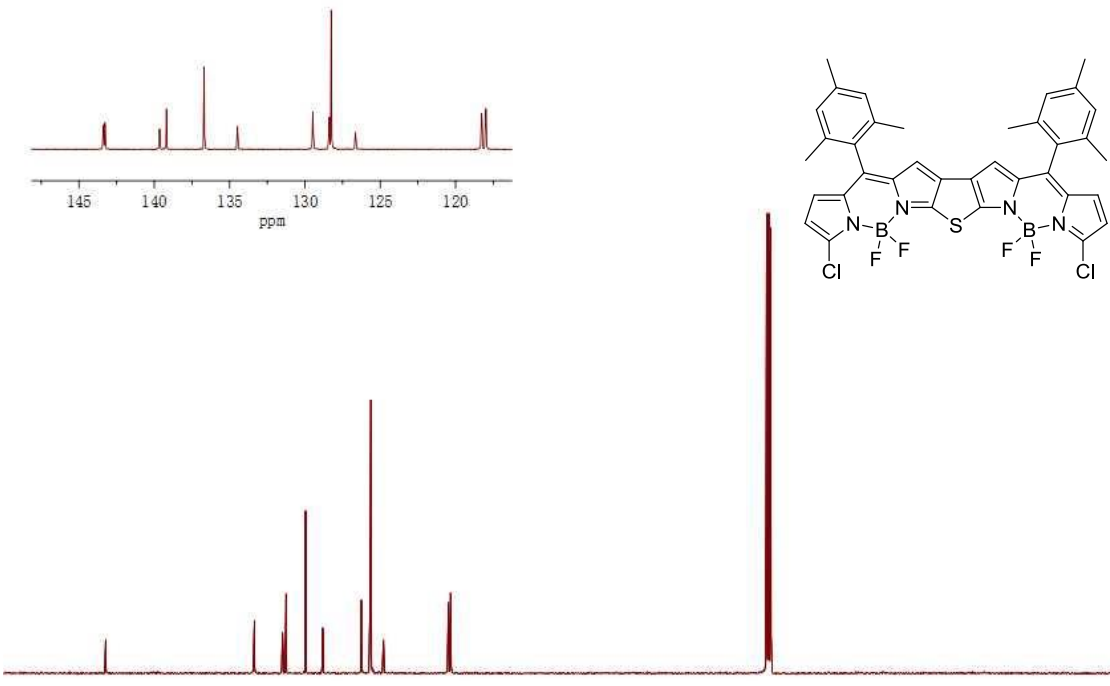

${ }^{13} \mathrm{C}$ NMR spectrum of FD2 $\left(\mathrm{CDCl}_{3}, 126 \mathrm{MHz}\right)$ 


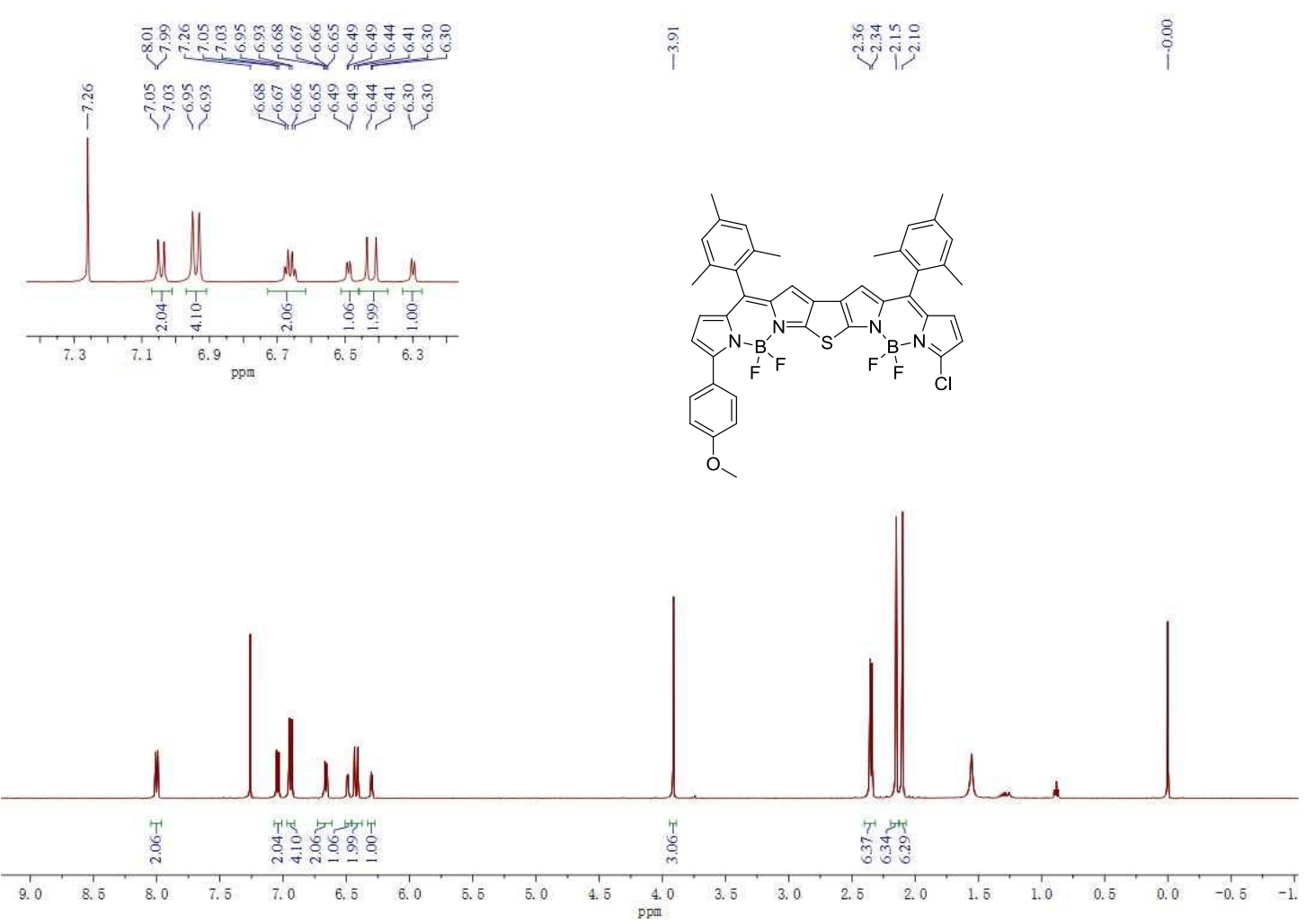

${ }^{1} \mathrm{H}$ NMR spectrum of $\mathbf{F D 3}\left(\mathrm{CDCl}_{3}, 500 \mathrm{MHz}\right)$
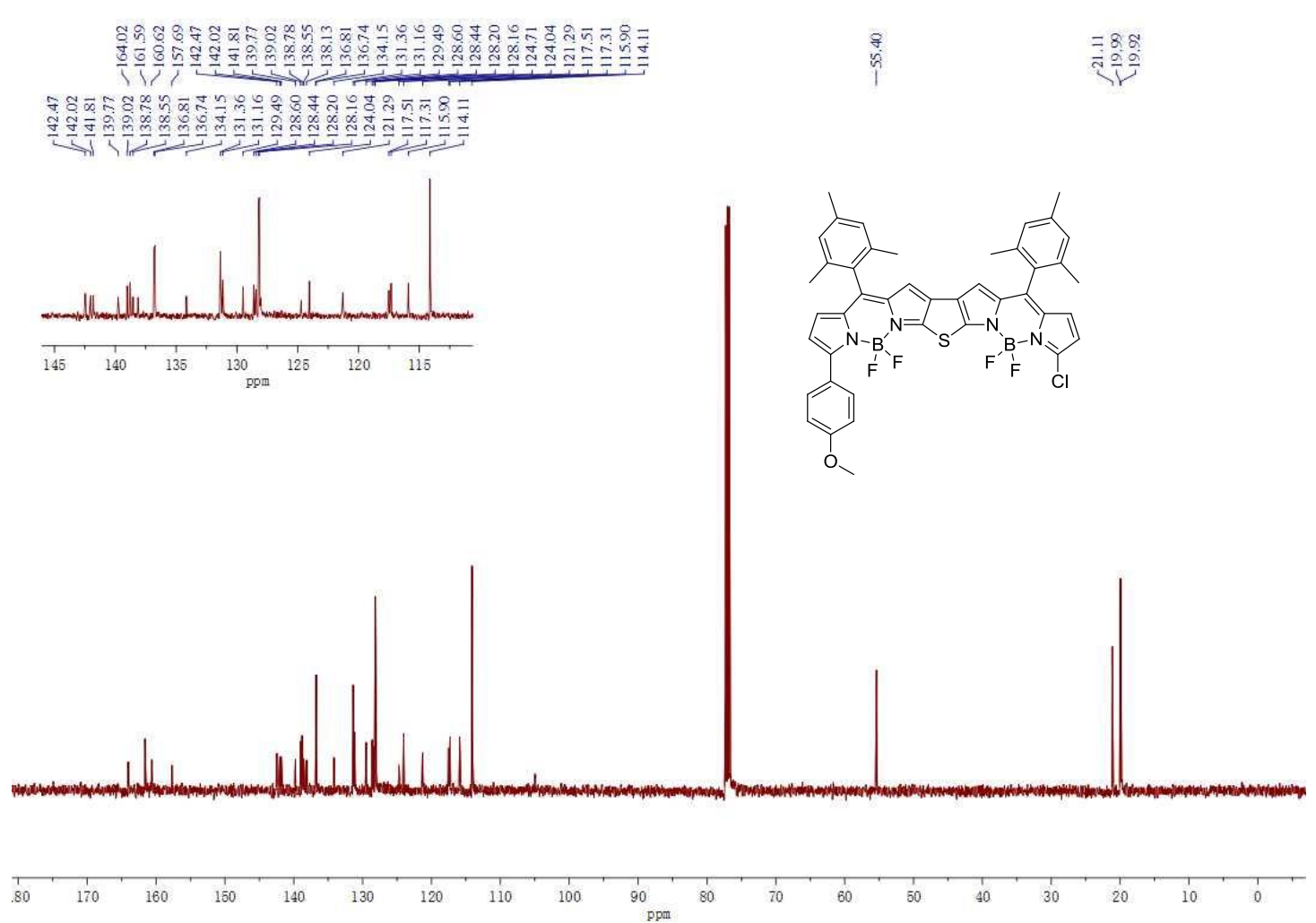

${ }^{13} \mathrm{C} \mathrm{NMR}$ spectrum of $\mathbf{F D 3}\left(\mathrm{CDCl}_{3}, 126 \mathrm{MHz}\right)$ 


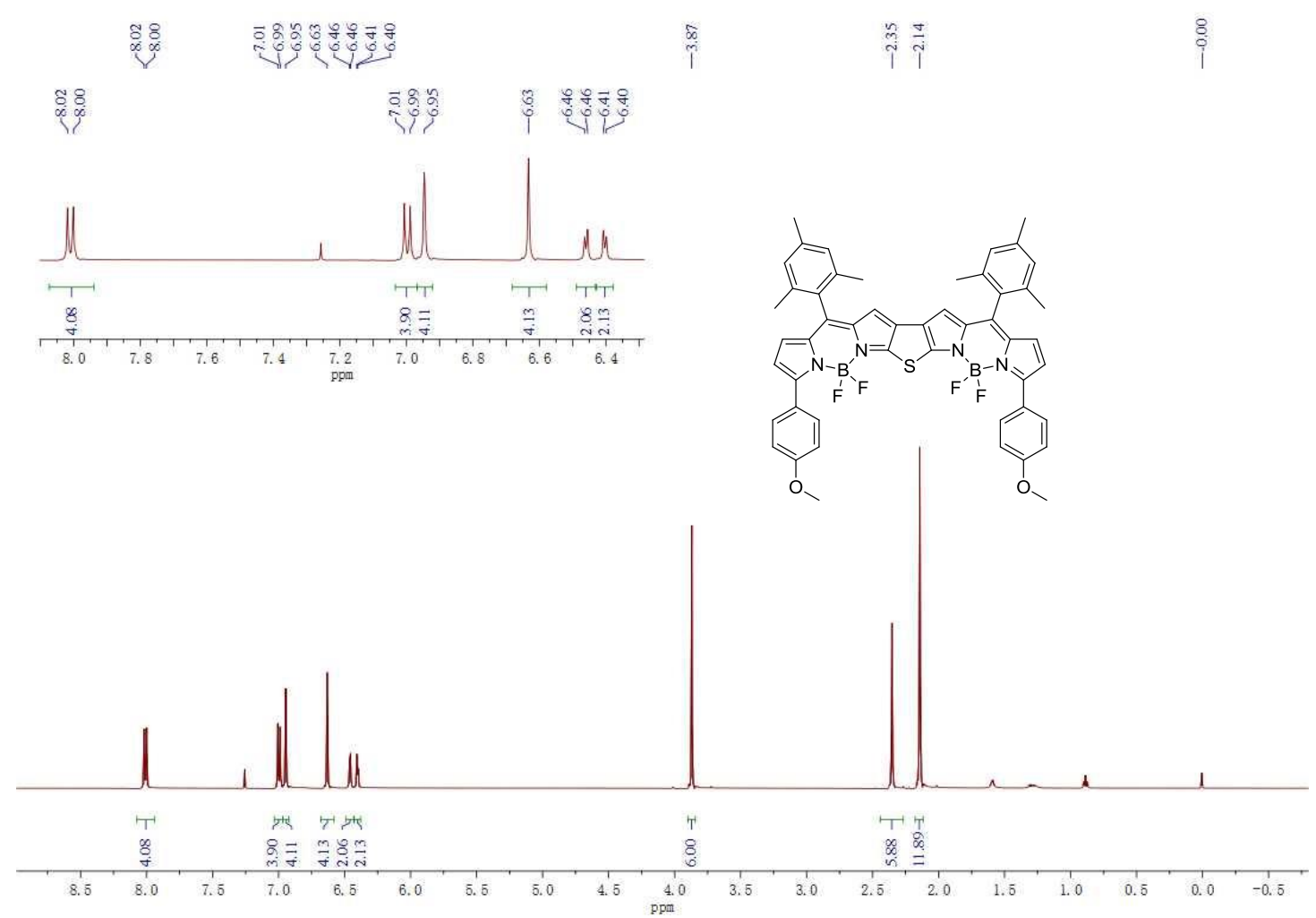

${ }^{1} \mathrm{H}$ NMR spectrum of FD4 $\left(\mathrm{CDCl}_{3}, 500 \mathrm{MHz}\right)$

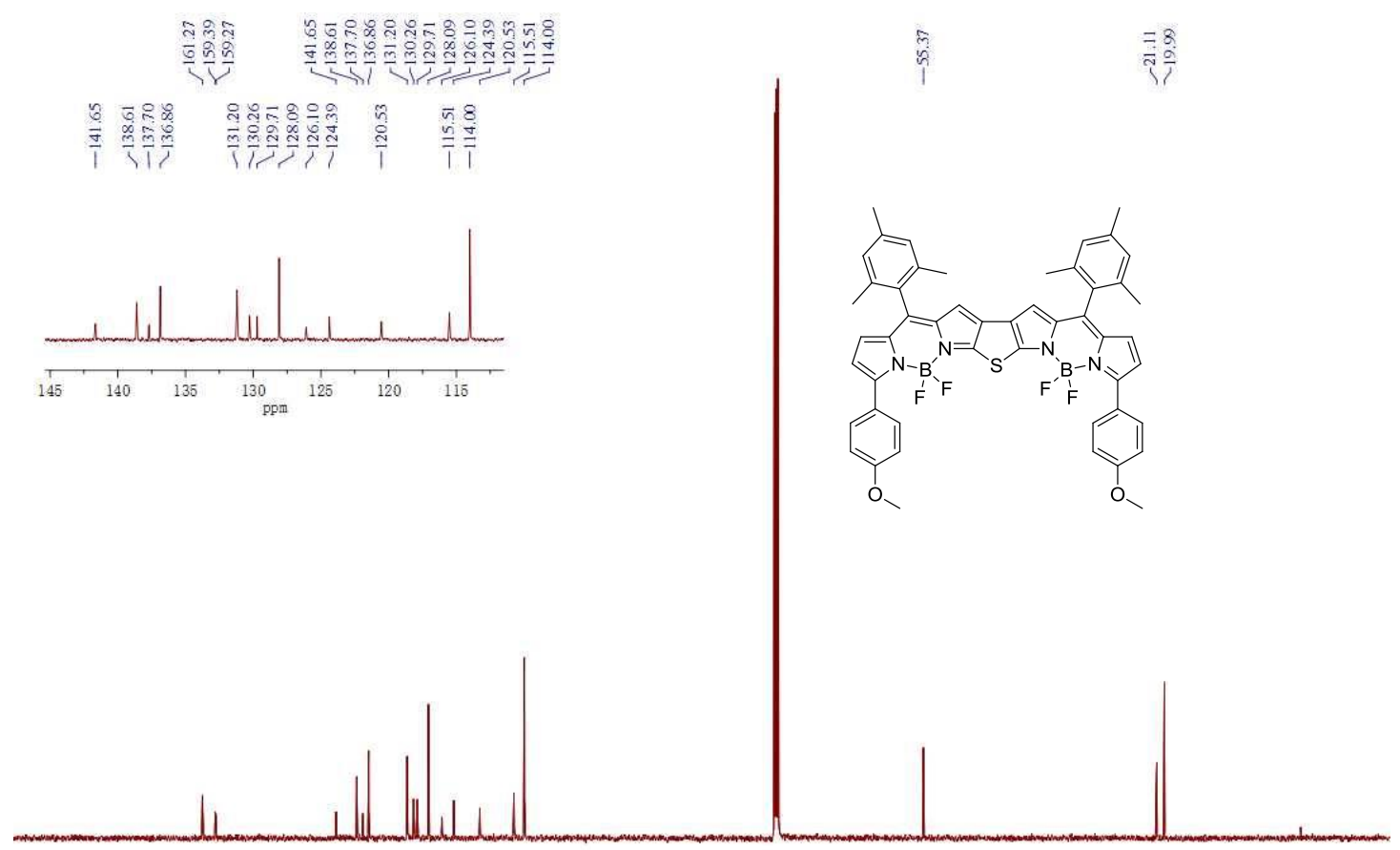

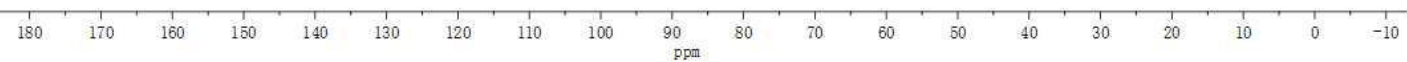

${ }^{13} \mathrm{C}$ NMR spectrum of FD4 $\left(\mathrm{CDCl}_{3}, 126 \mathrm{MHz}\right)$ 

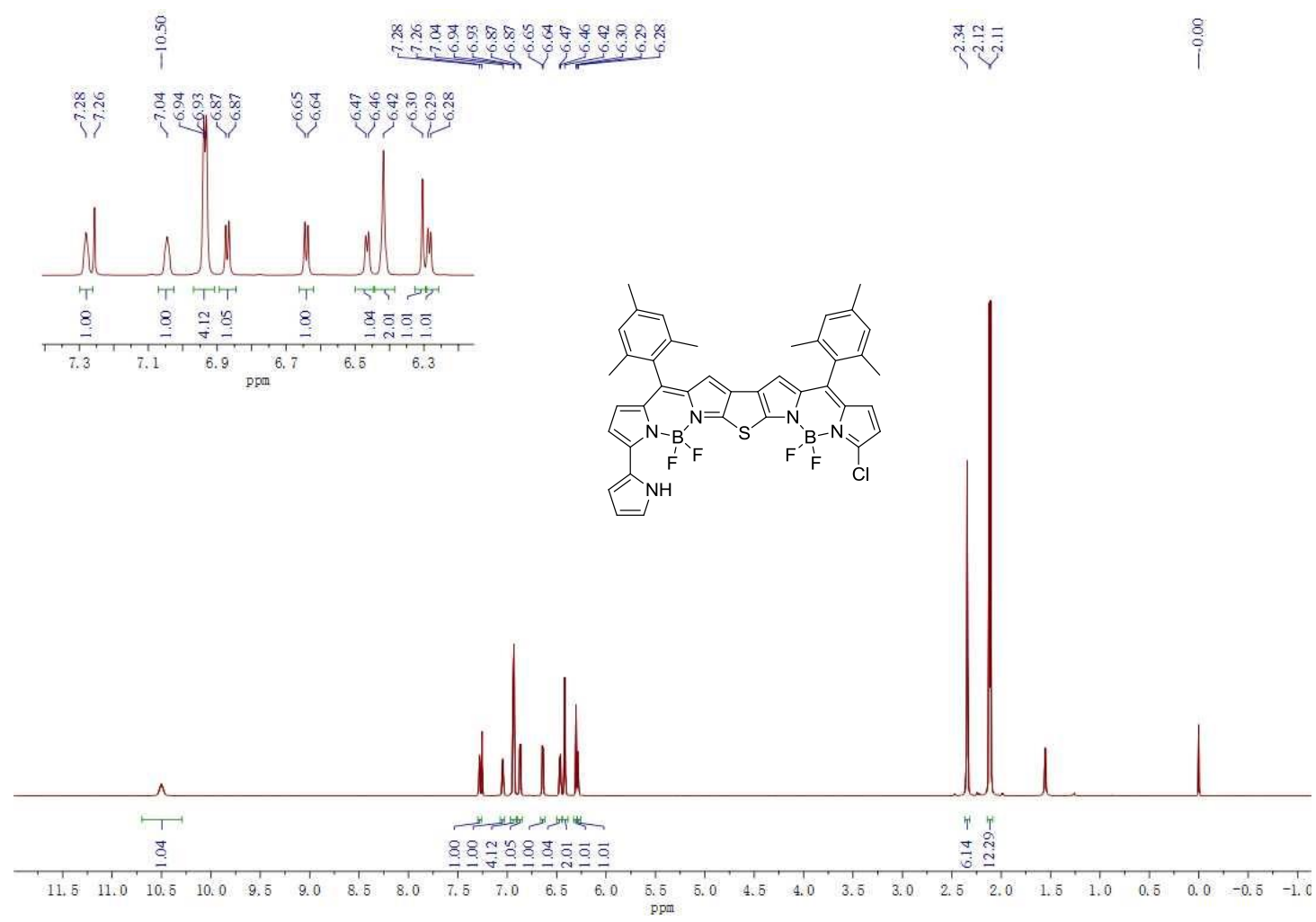

${ }^{1} \mathrm{H}$ NMR spectrum of $\mathbf{F D 5}\left(\mathrm{CDCl}_{3}, 500 \mathrm{MHz}\right)$
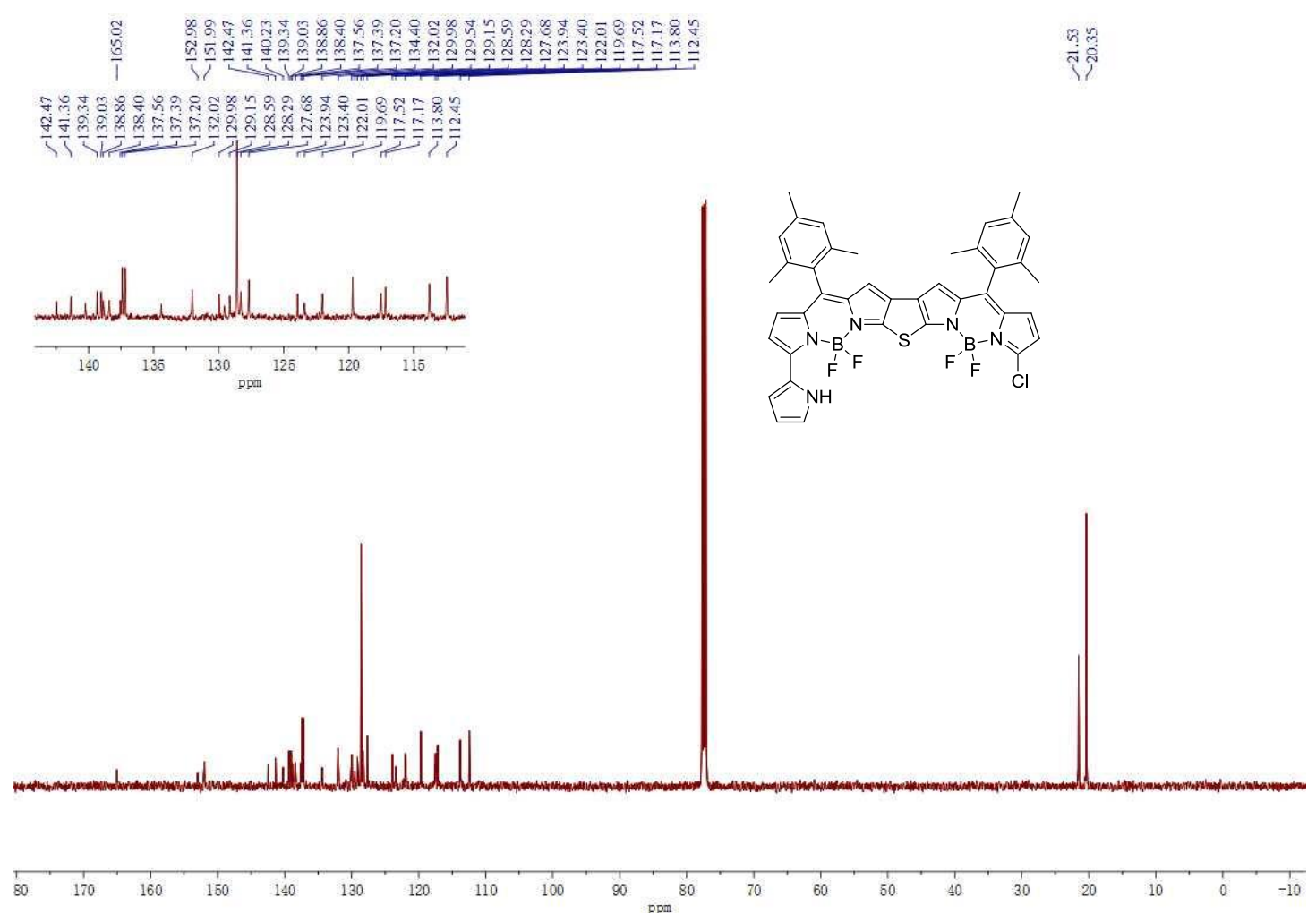

${ }^{13} \mathrm{C}$ NMR spectrum of FD5 $\left(\mathrm{CDCl}_{3}, 126 \mathrm{MHz}\right)$ 


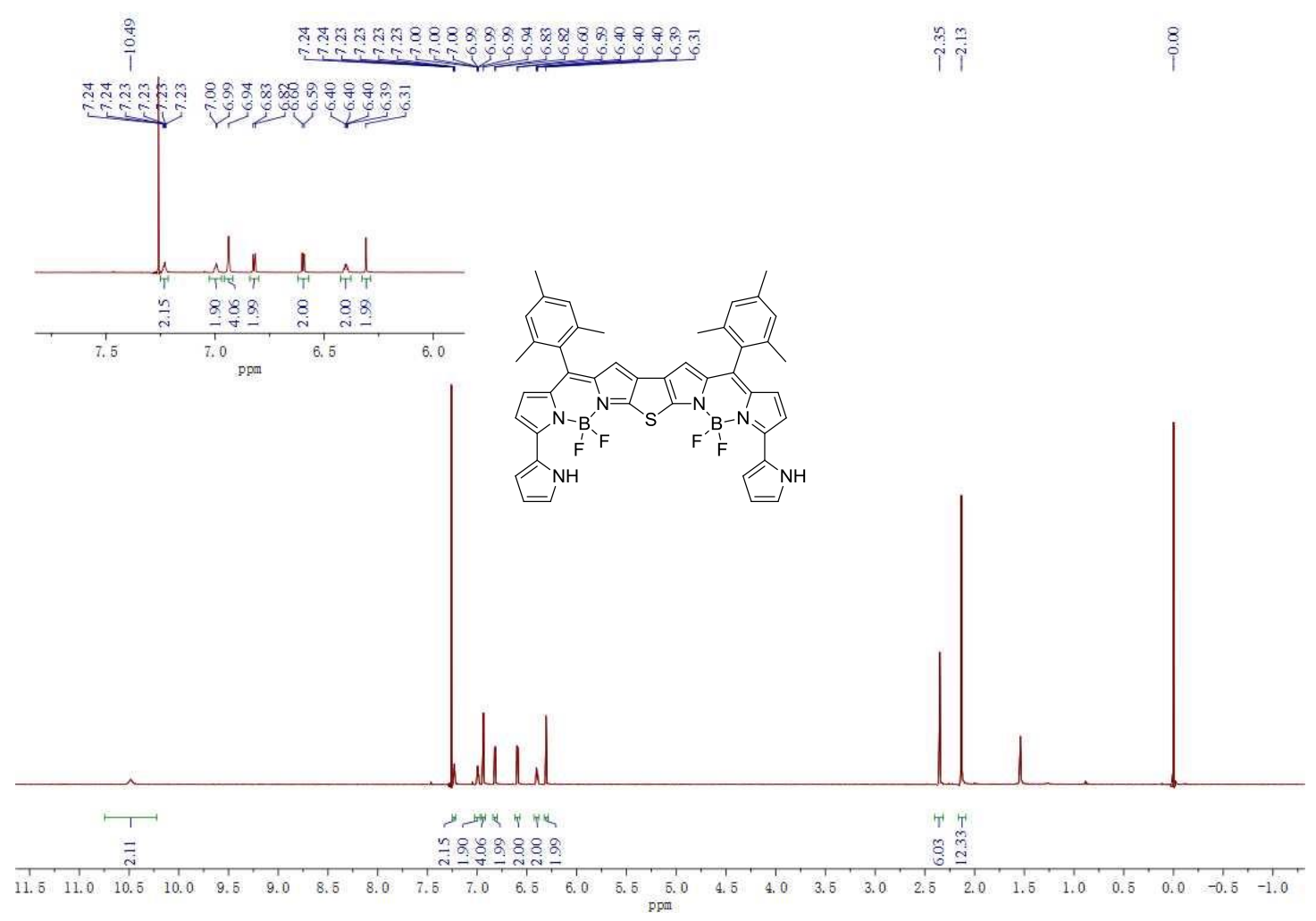

${ }^{1} \mathrm{H}$ NMR spectrum of $\mathbf{F D 6}\left(\mathrm{CDCl}_{3}, 500 \mathrm{MHz}\right)$
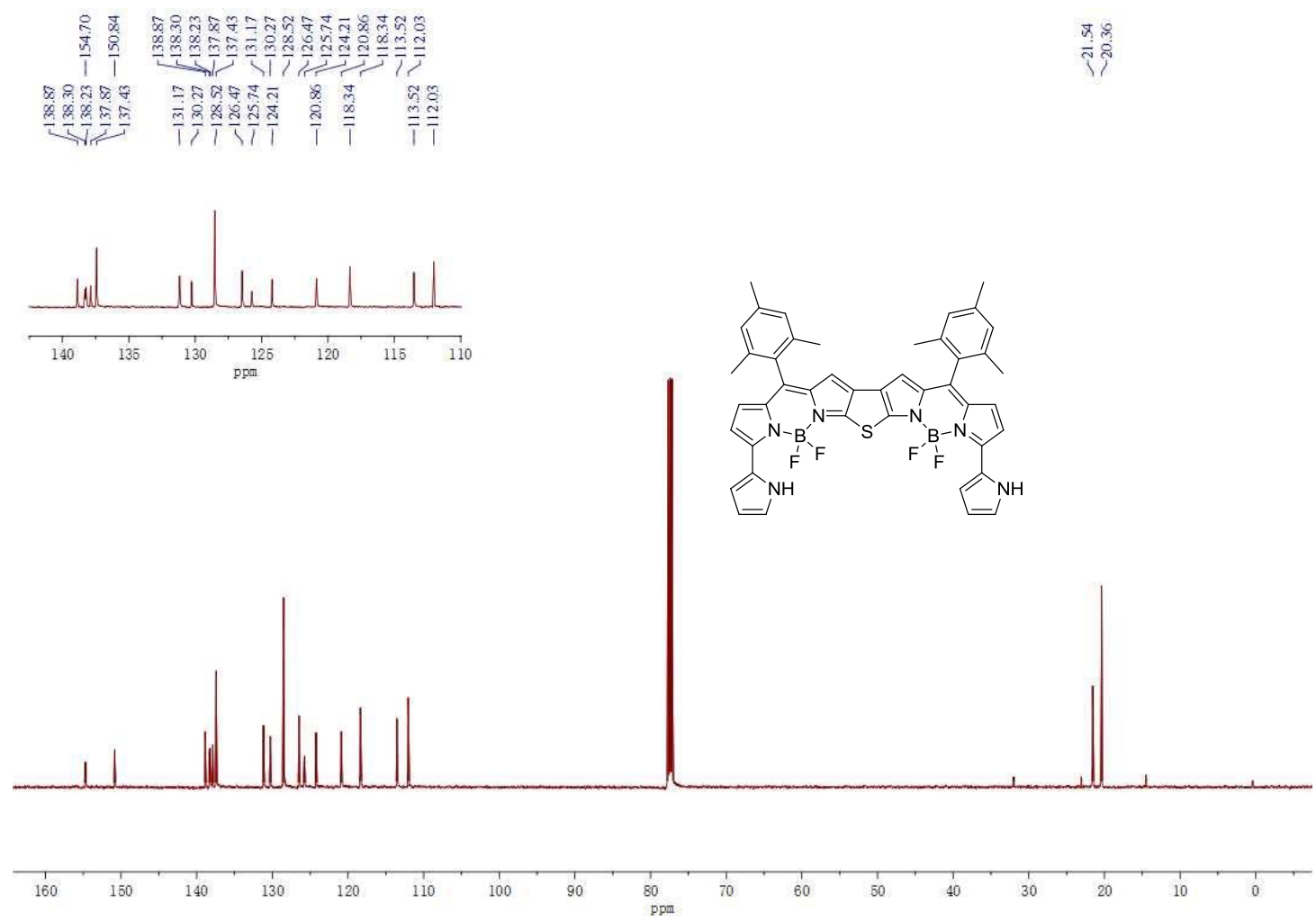

${ }^{13} \mathrm{C} \mathrm{NMR}$ spectrum of $\mathbf{F D 6}\left(\mathrm{CDCl}_{3}, 126 \mathrm{MHz}\right)$ 


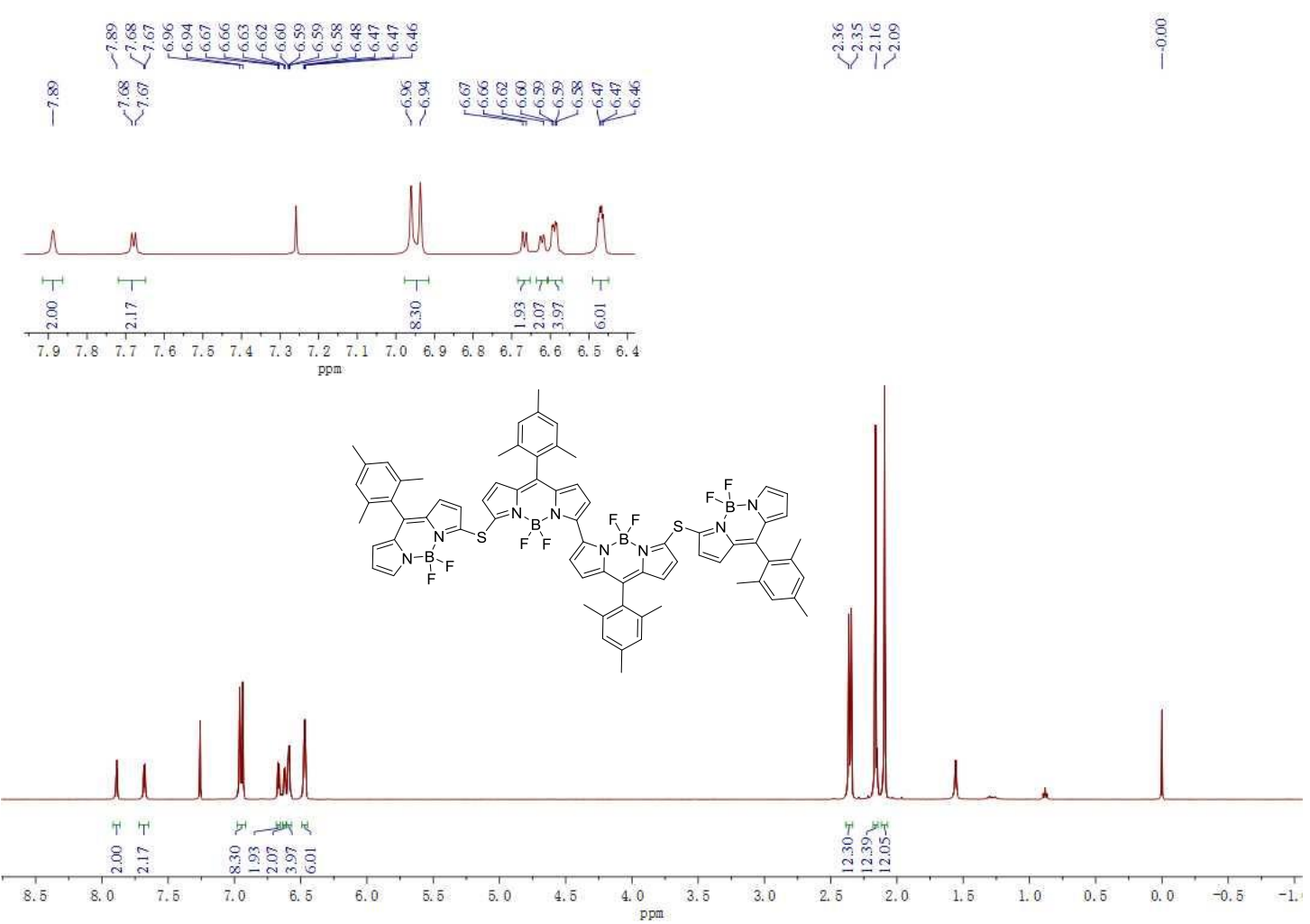

${ }^{1} \mathrm{H}$ NMR spectrum of $\mathbf{T 1}\left(\mathrm{CDCl}_{3}, 500 \mathrm{MHz}\right)$
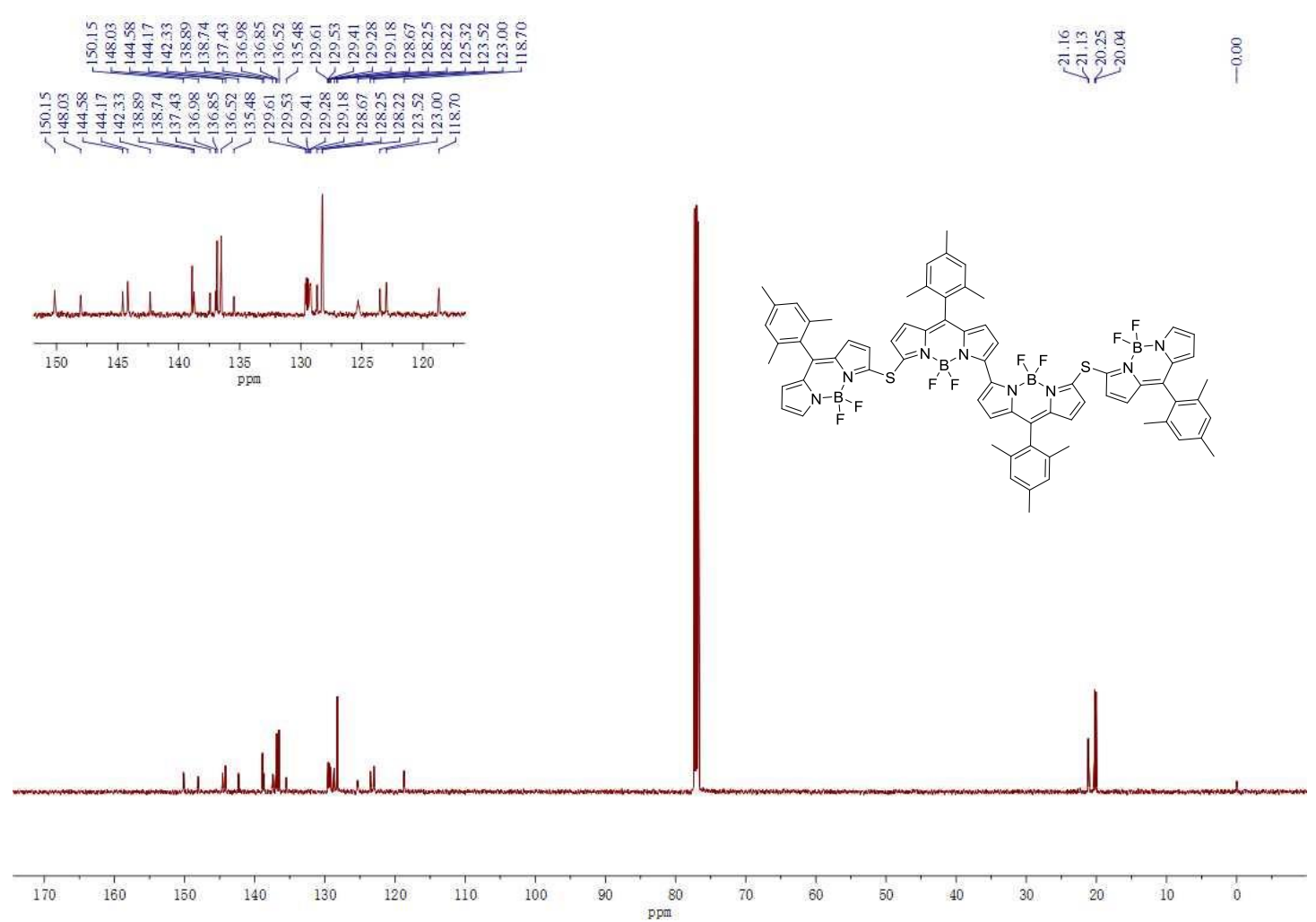

${ }^{13} \mathrm{C}$ NMR spectrum of $\mathbf{T 1}\left(\mathrm{CDCl}_{3}, 126 \mathrm{MHz}\right)$ 


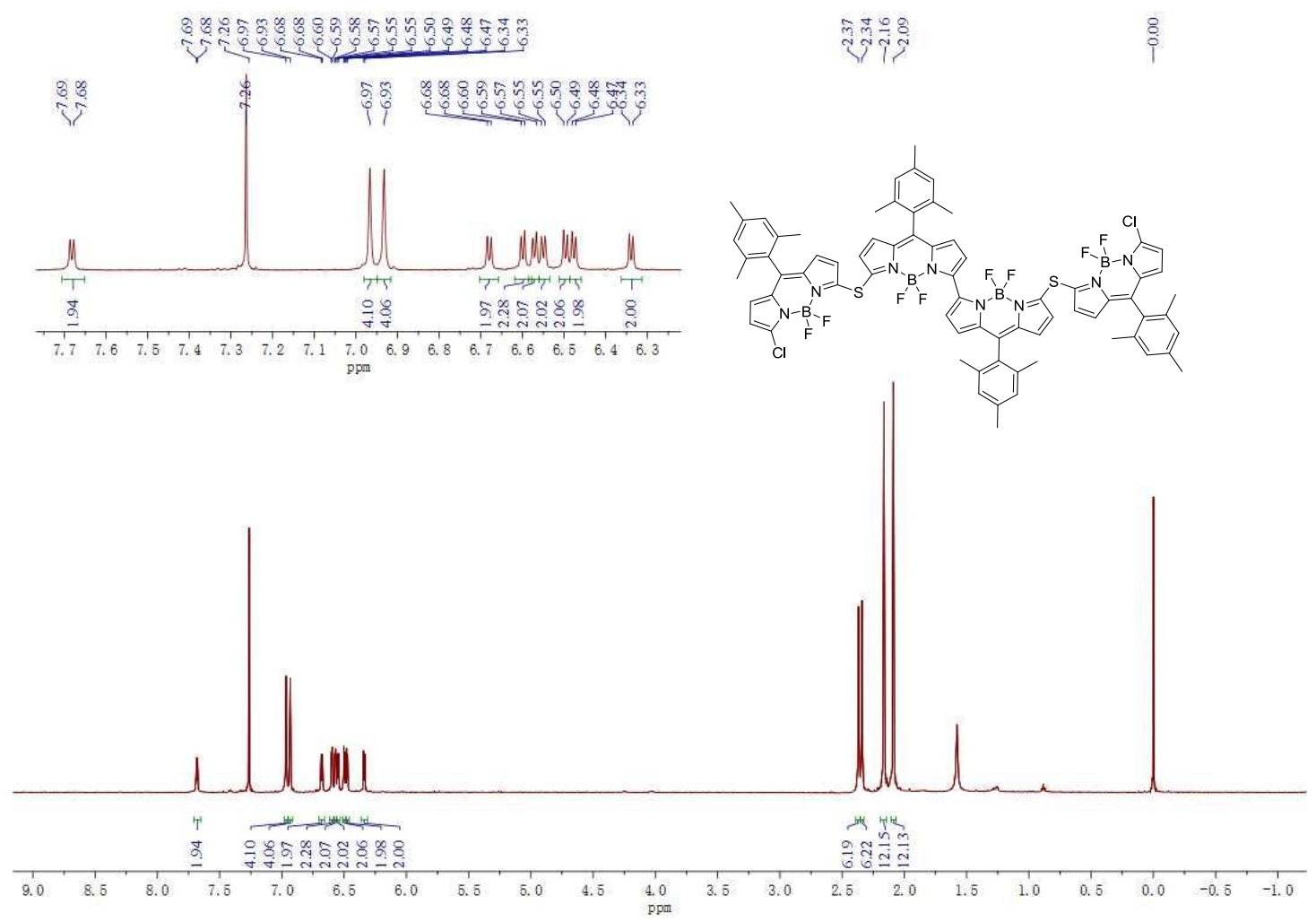

${ }^{1} \mathrm{H}$ NMR spectrum of $\mathbf{T} 2\left(\mathrm{CDCl}_{3}, 500 \mathrm{MHz}\right)$
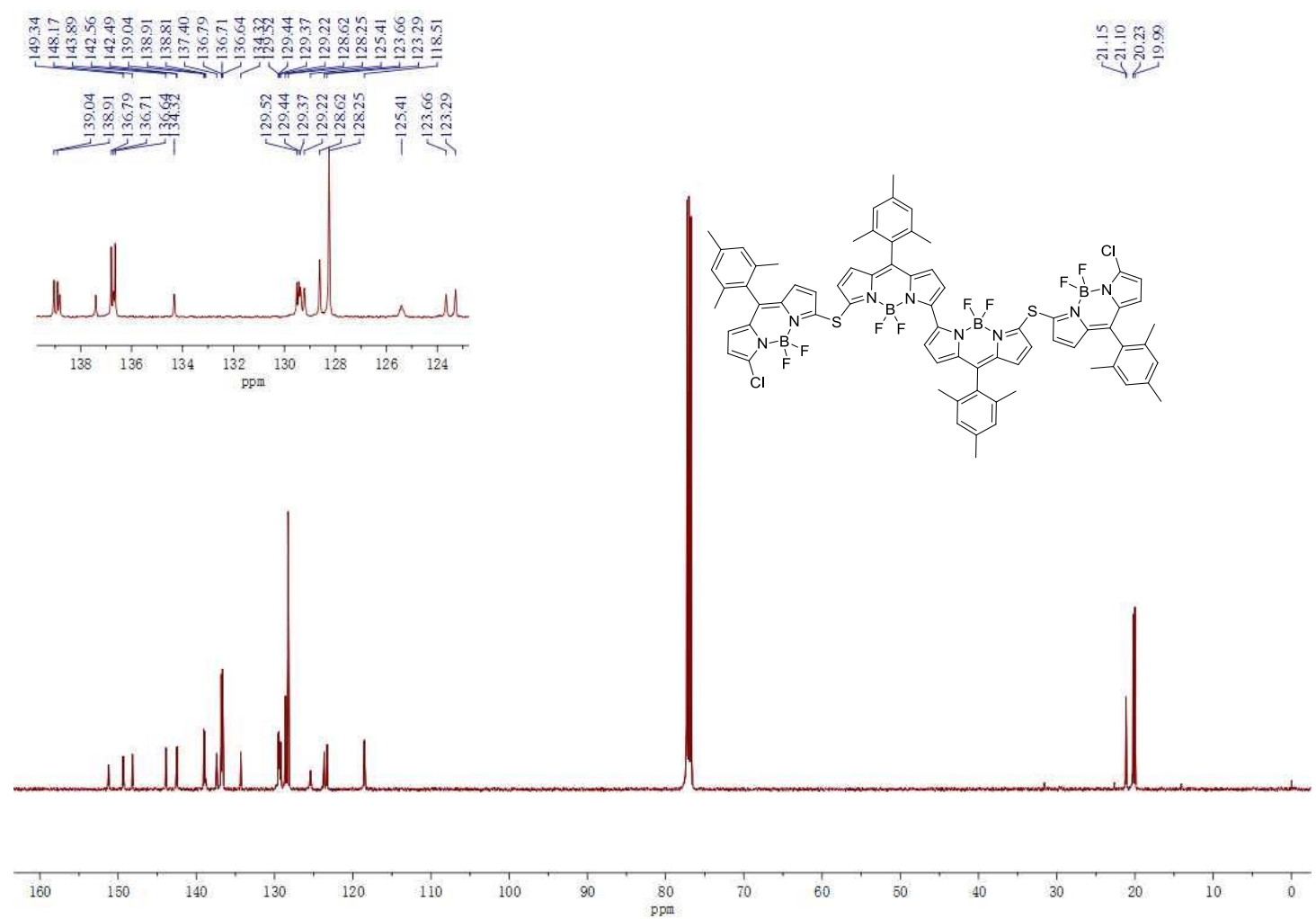

${ }^{13} \mathrm{C}$ NMR spectrum of $\mathbf{T} 2\left(\mathrm{CDCl}_{3}, 126 \mathrm{MHz}\right)$ 


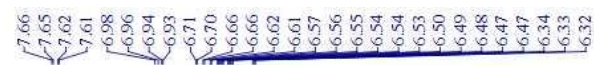

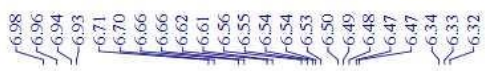
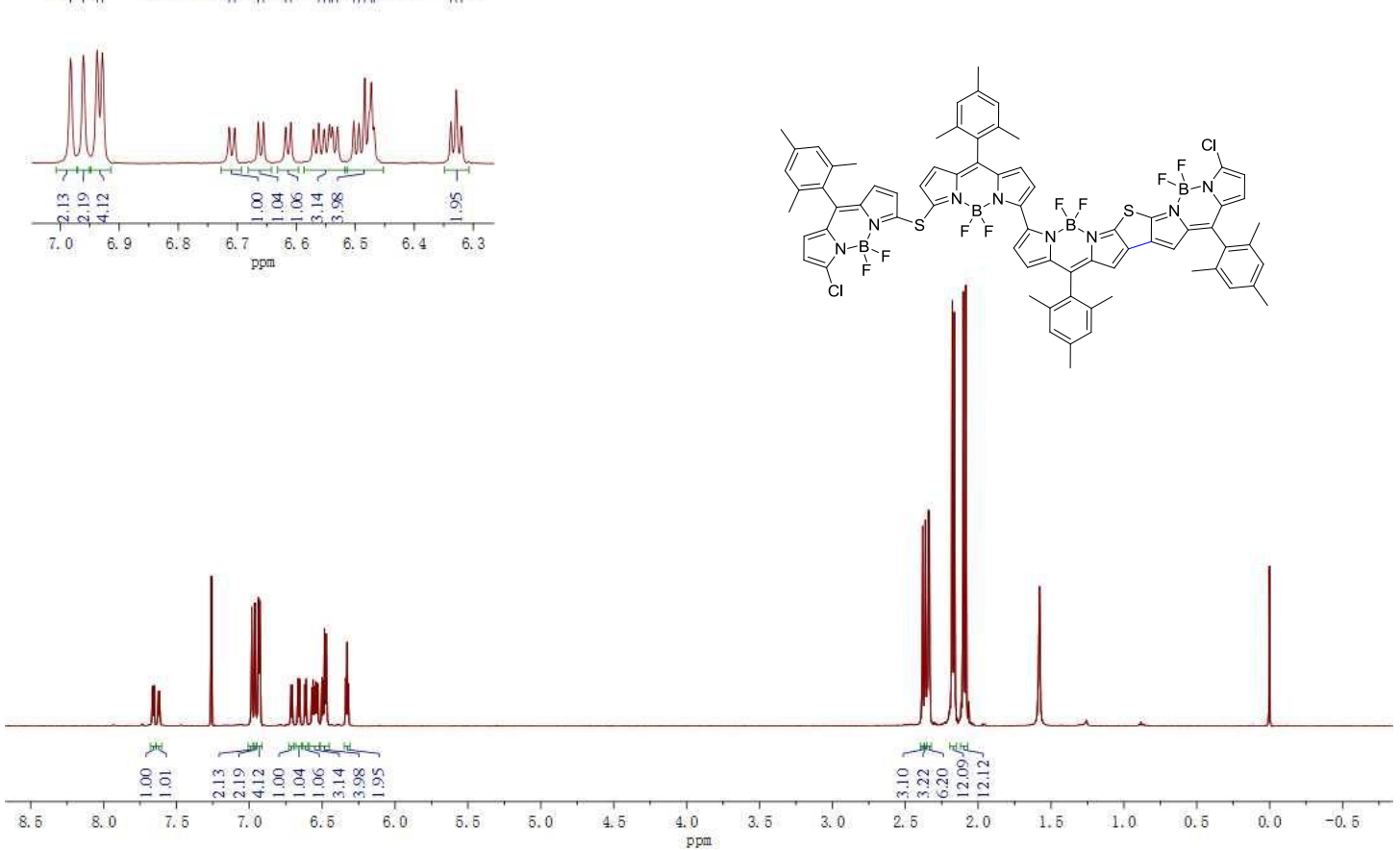

${ }^{1} \mathrm{H}$ NMR spectrum of $\mathbf{T 3}\left(\mathrm{CDCl}_{3}, 500 \mathrm{MHz}\right)$

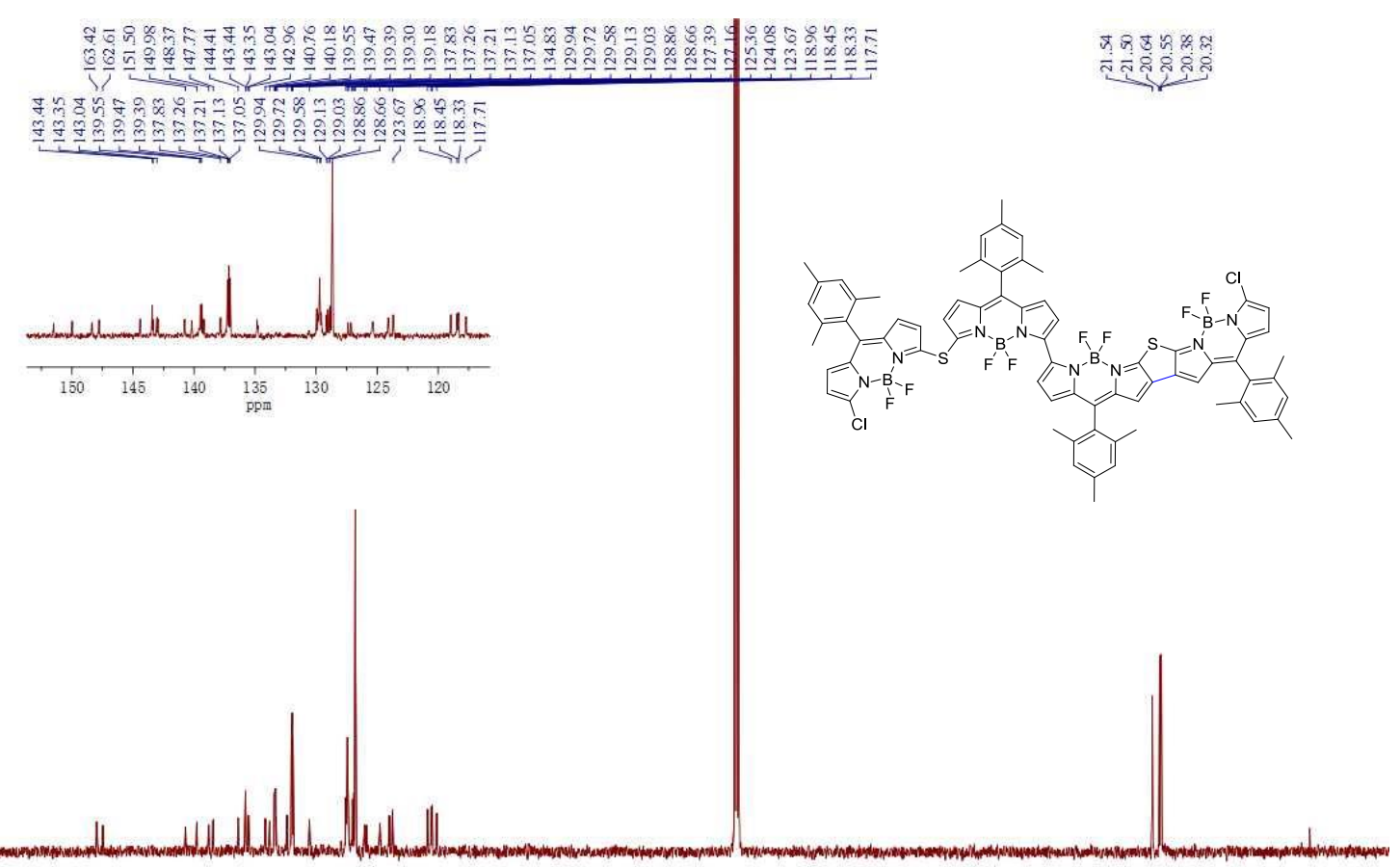

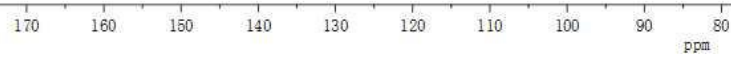

${ }^{13} \mathrm{C}$ NMR spectrum of $\mathbf{T 3}\left(\mathrm{CDCl}_{3}, 126 \mathrm{MHz}\right)$ 


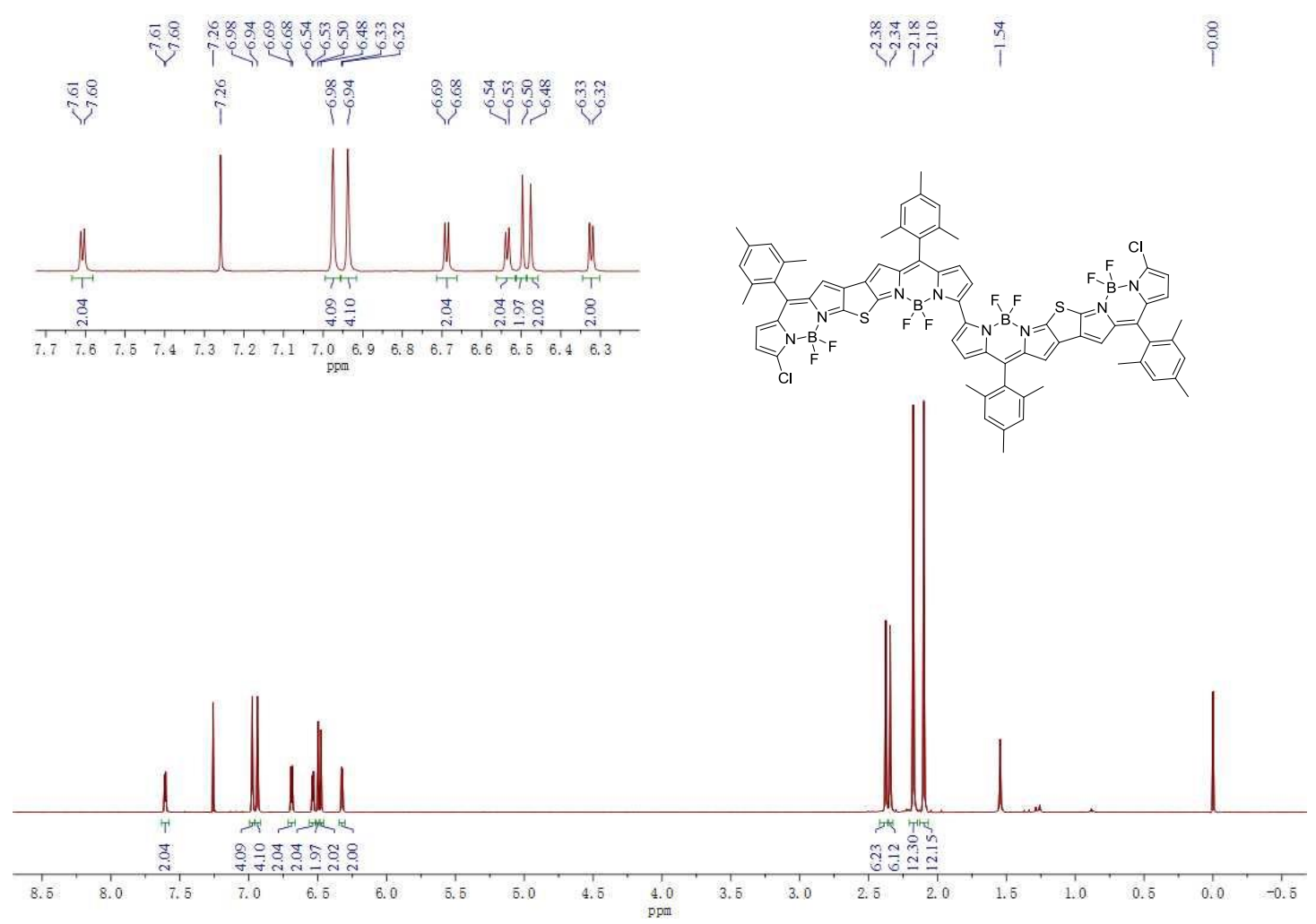

${ }^{1} \mathrm{H}$ NMR spectrum of $\mathbf{T} 4\left(\mathrm{CDCl}_{3}, 500 \mathrm{MHz}\right)$
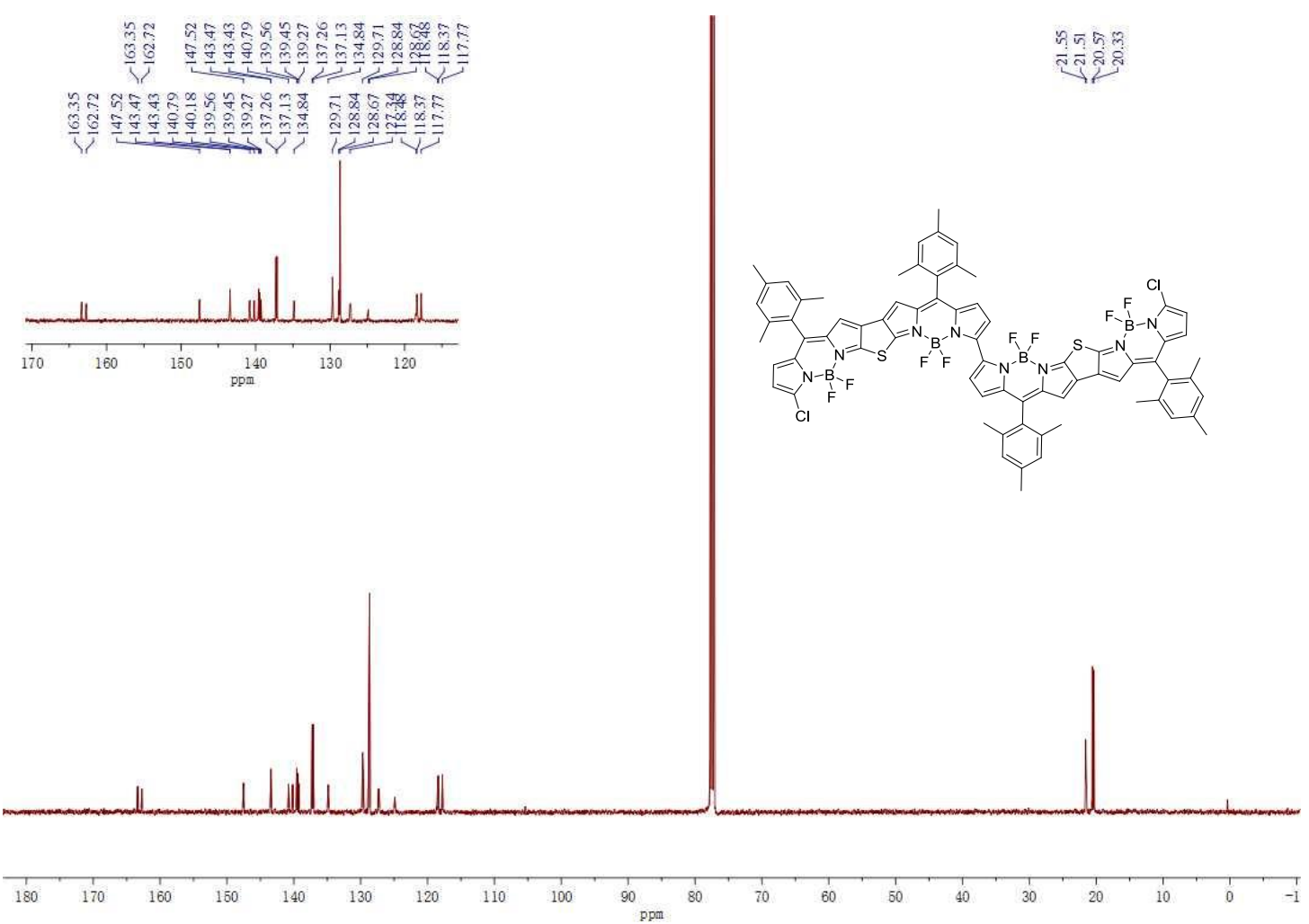

${ }^{13} \mathrm{C}$ NMR spectrum of $\mathbf{T} 4\left(\mathrm{CDCl}_{3}, 126 \mathrm{MHz}\right)$ 
9. High resolution mass spectroscopes for all new compound

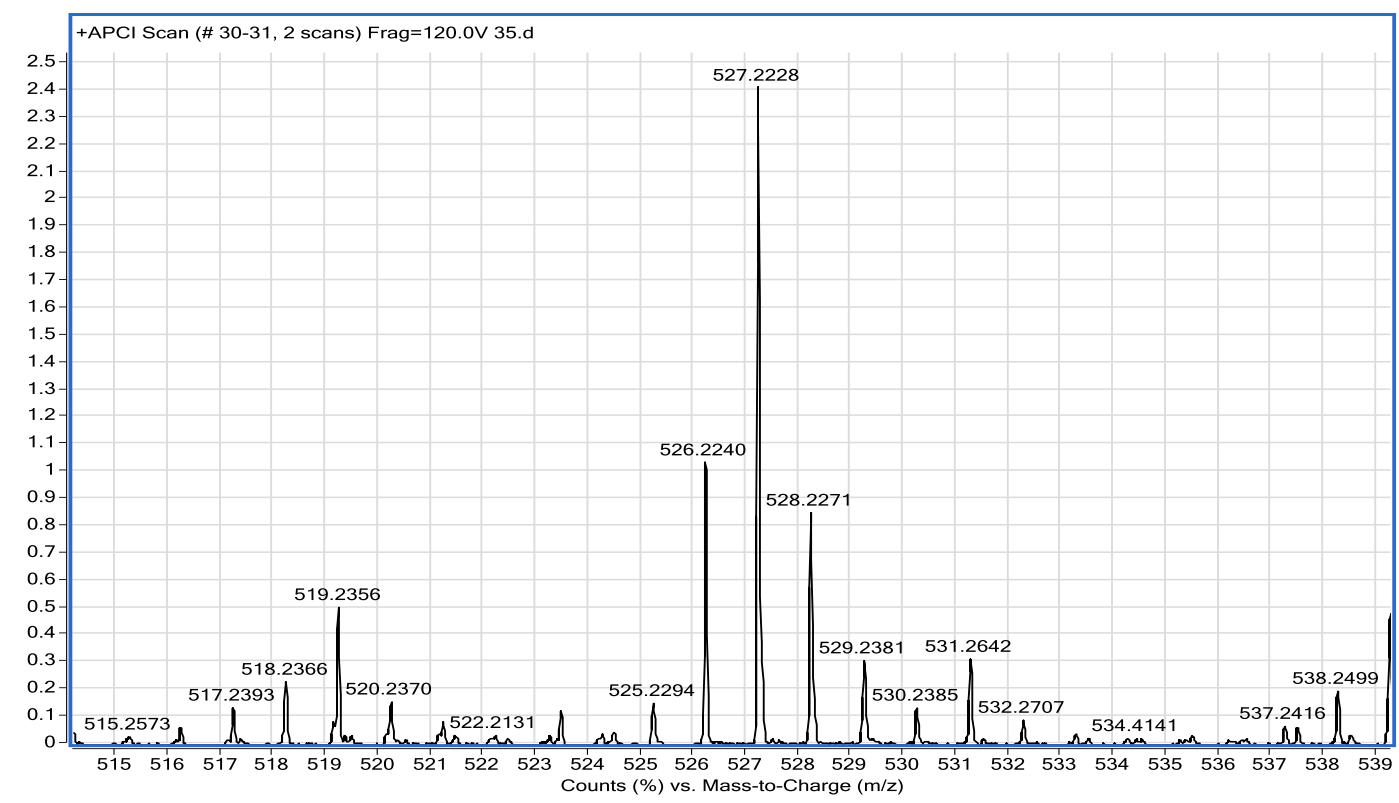

\section{HRMS for D1}

78_180828155926 \#19 RT: 0.25 AV: 1 NL: 7.54E7

T: FTMS + p ESI Full ms [150.00-2000.00]

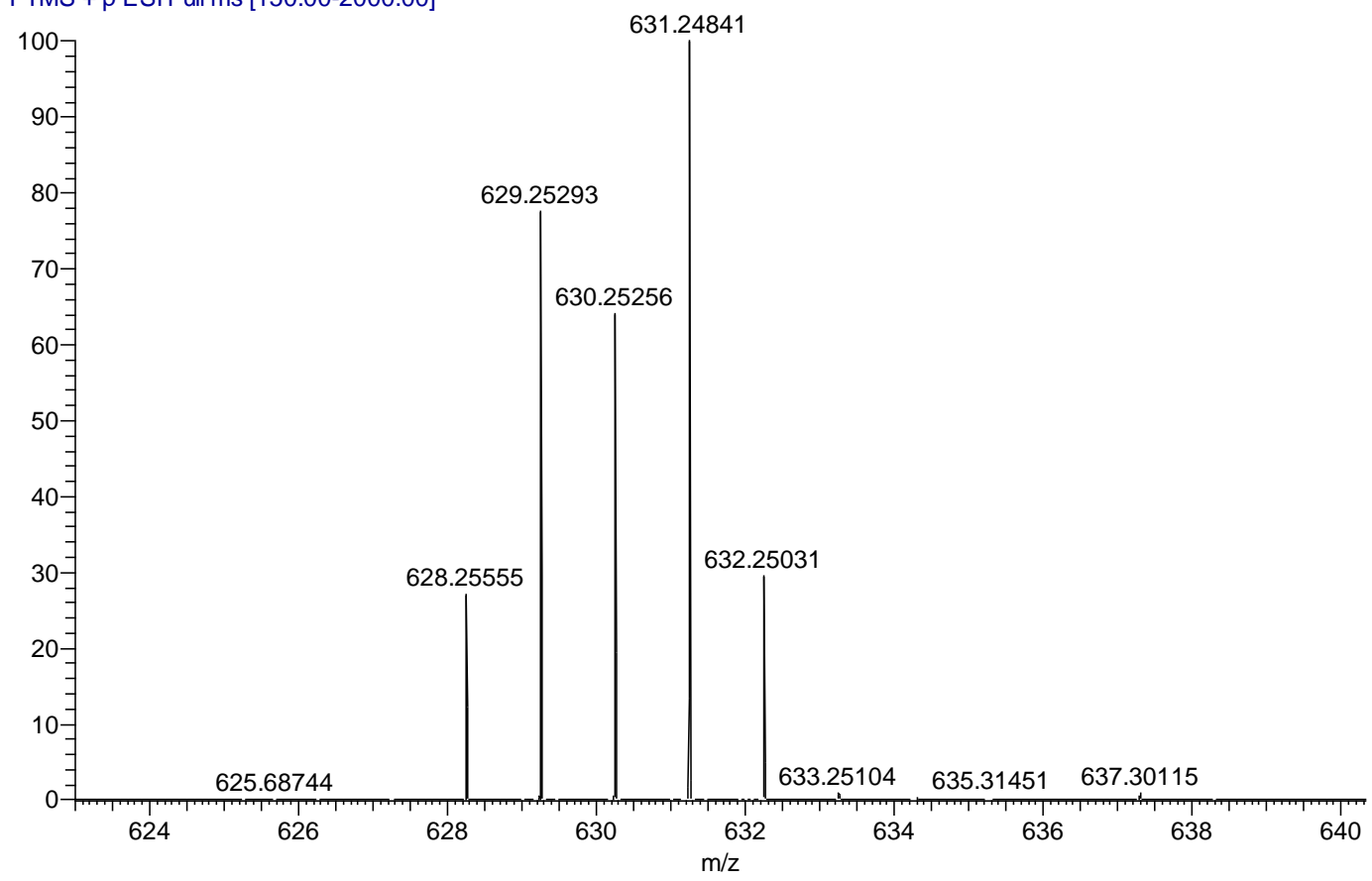

HRMS for D2 
79 \#31 RT: 0.42 AV: 1 NL: 1.87E6

T: FTMS + p ESIFull ms [150.00-2000.00]

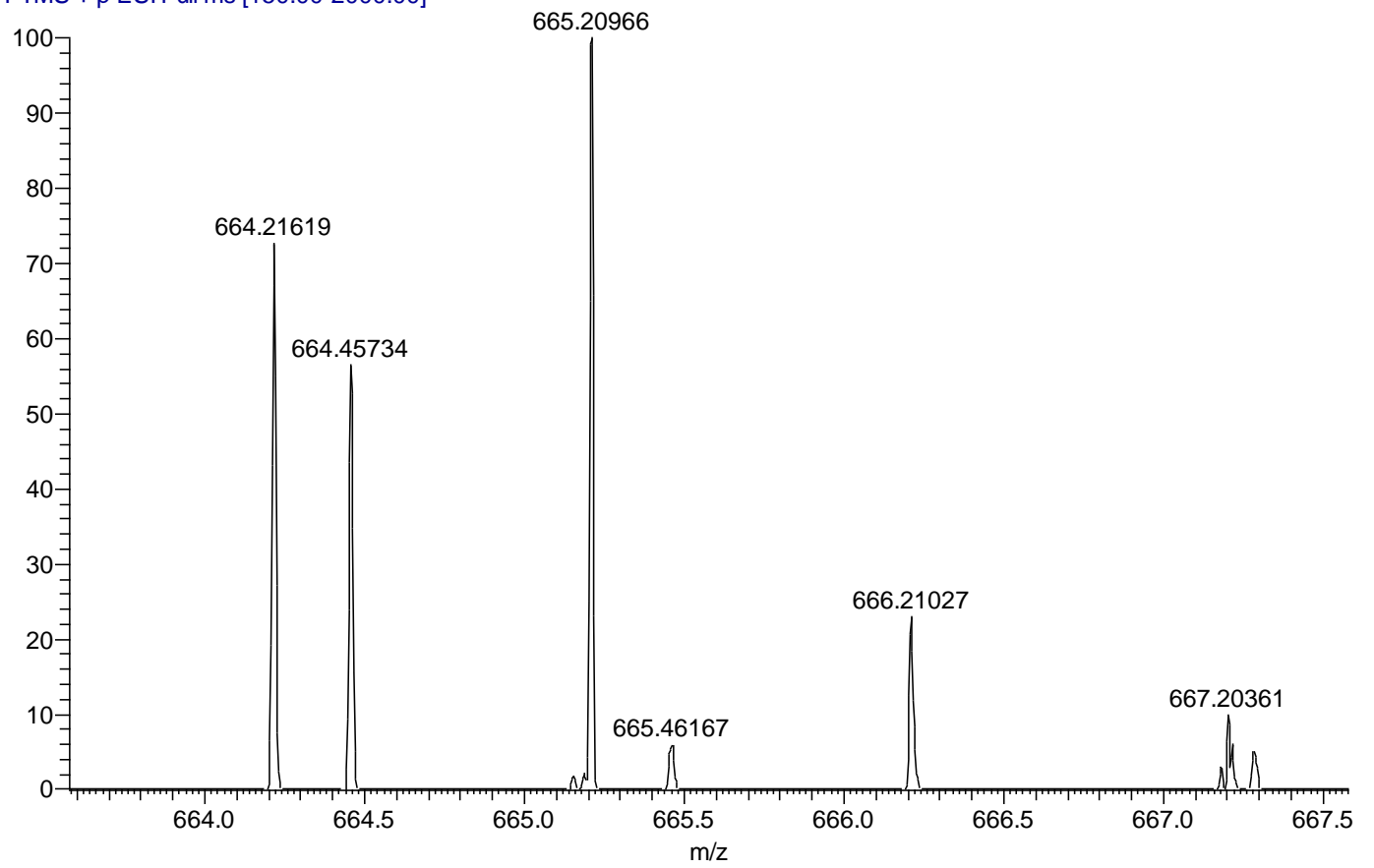

HRMS for D3

80 \#17 RT: 0.22 AV: 1 NL: 2.03E6

T: FTMS + p ESI Full ms [150.00-2000.00]

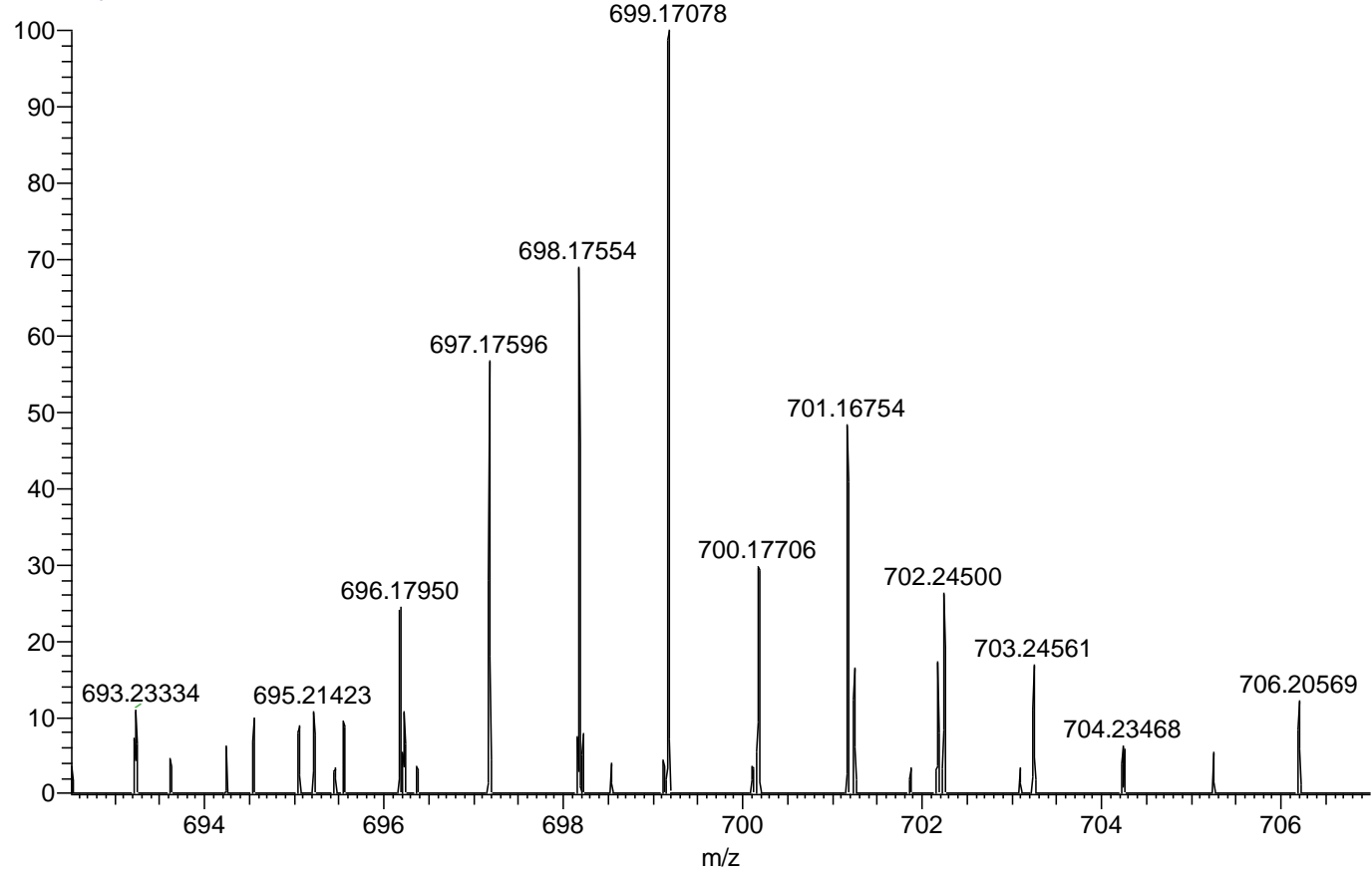

HRMS for D4 


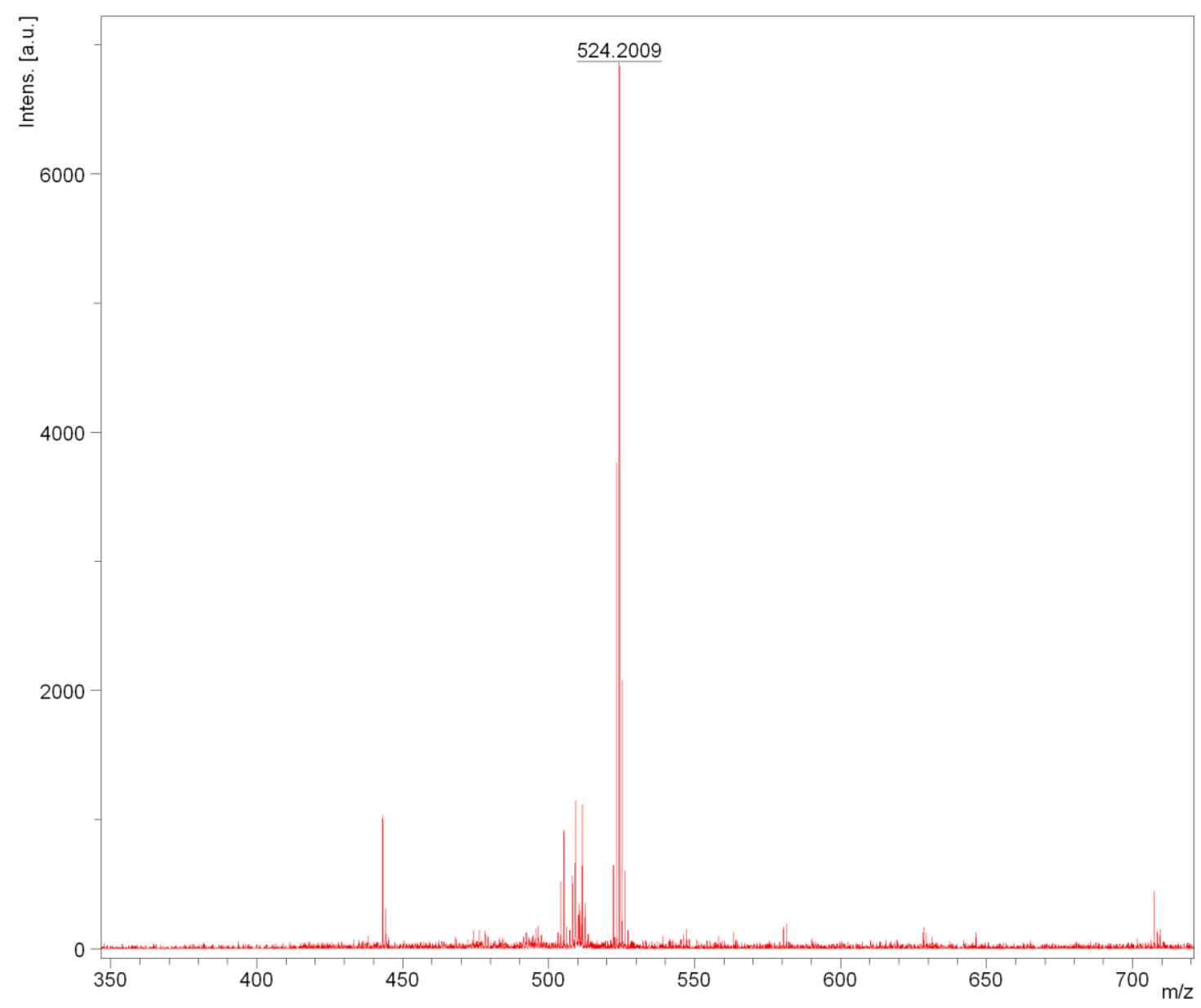

HRMS for FD1

82_180518162723 \#16 RT: 0.22 AV: 1 NL: $5.85 E 5$ T: FTMS + p ESI Full ms [150.00-2000.00]

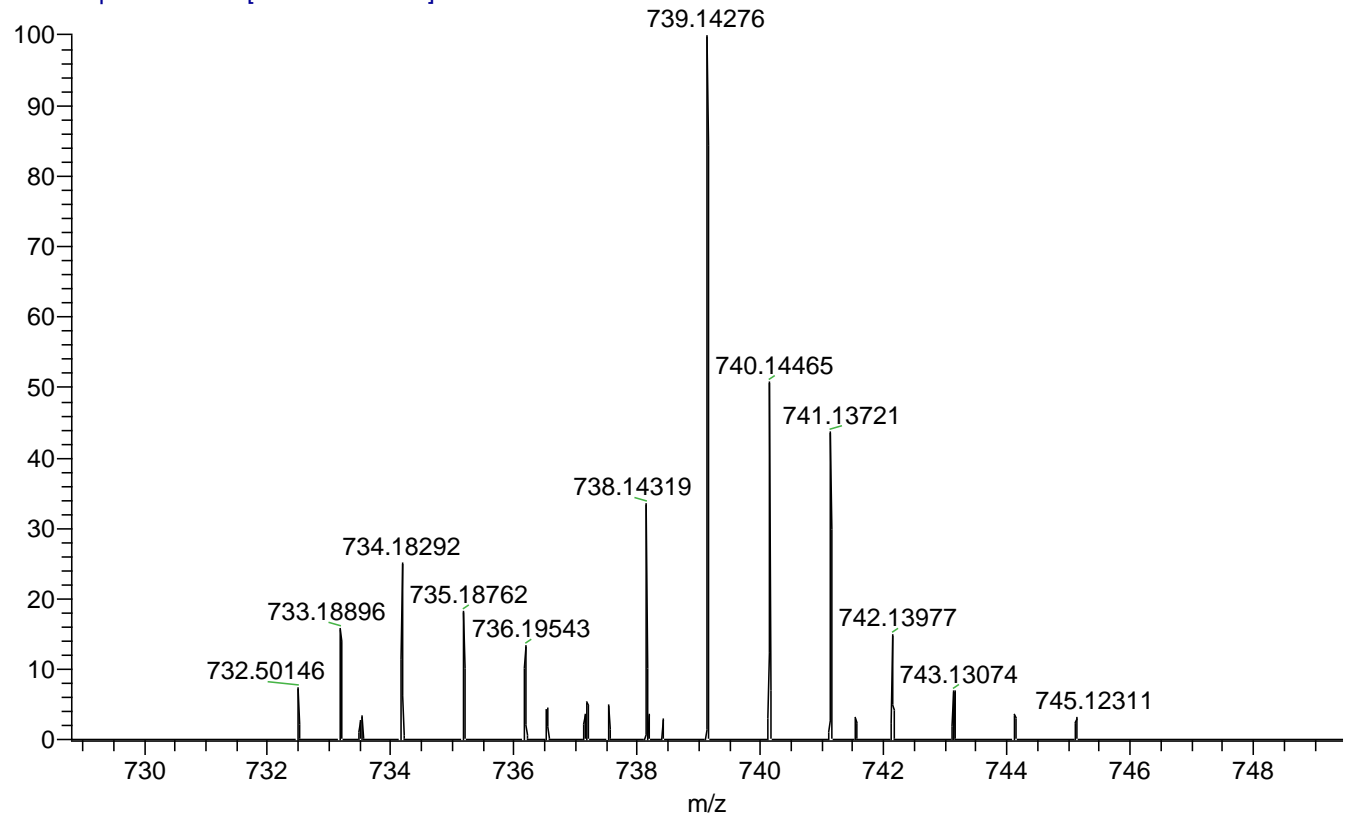

HRMS for FD2 


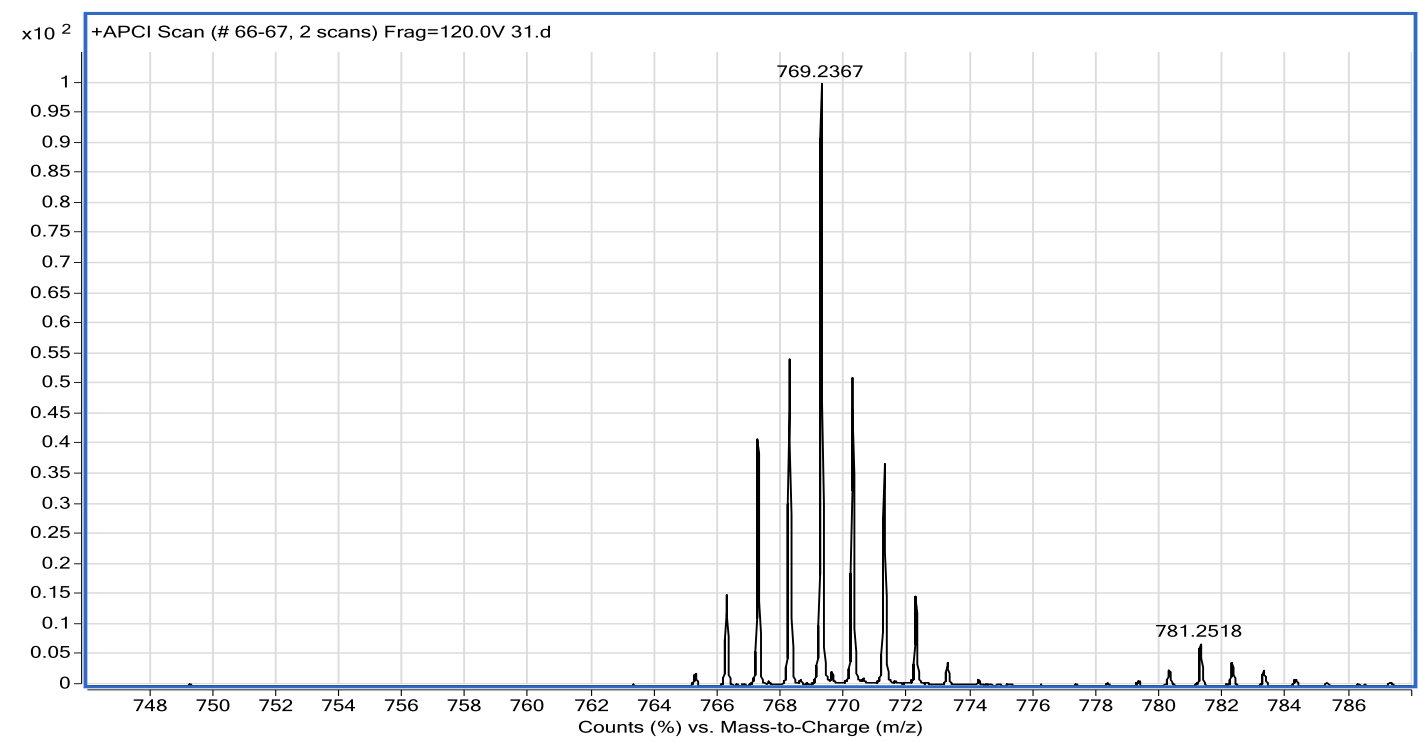

HRMS for FD3

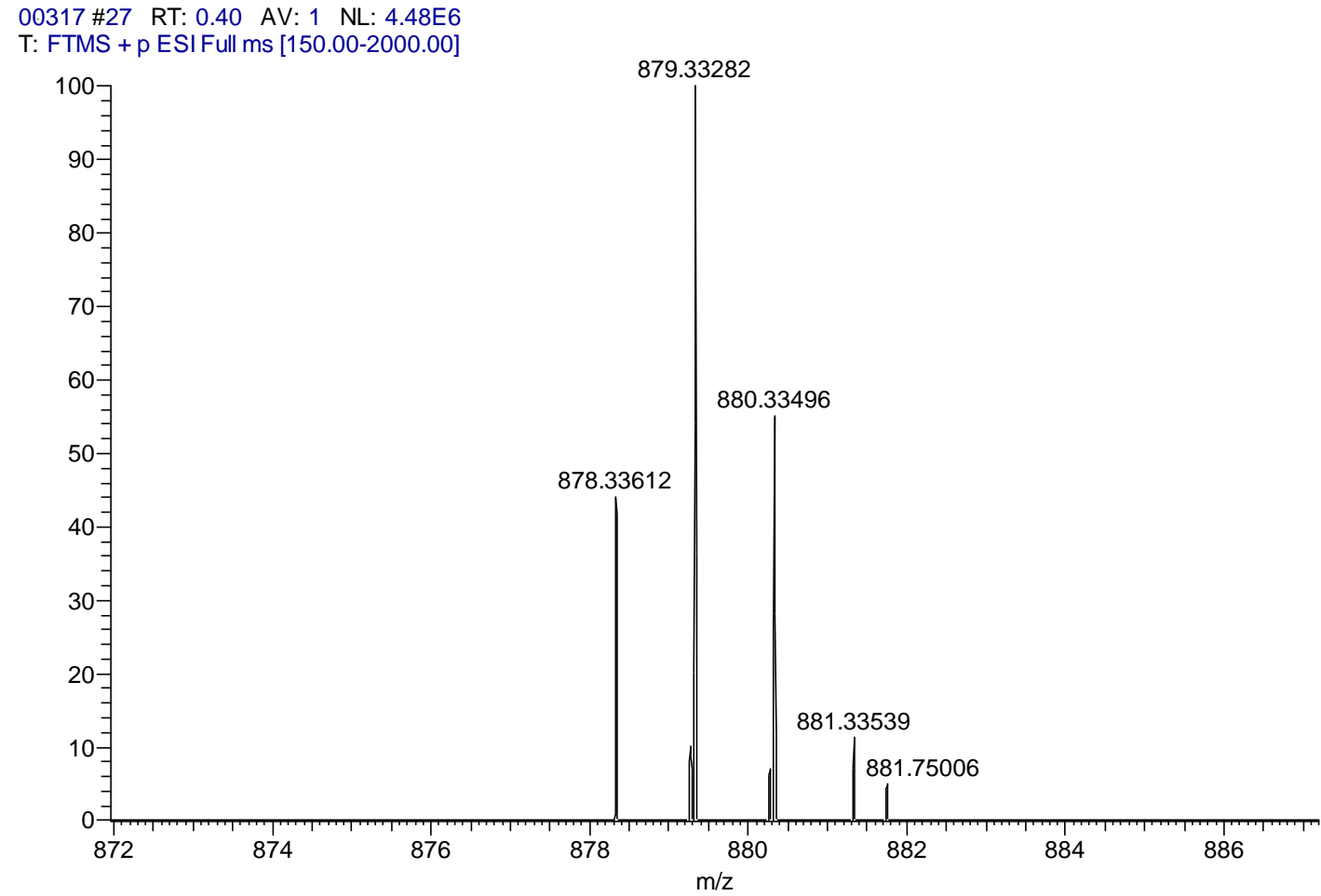

\section{HRMS for FD4}




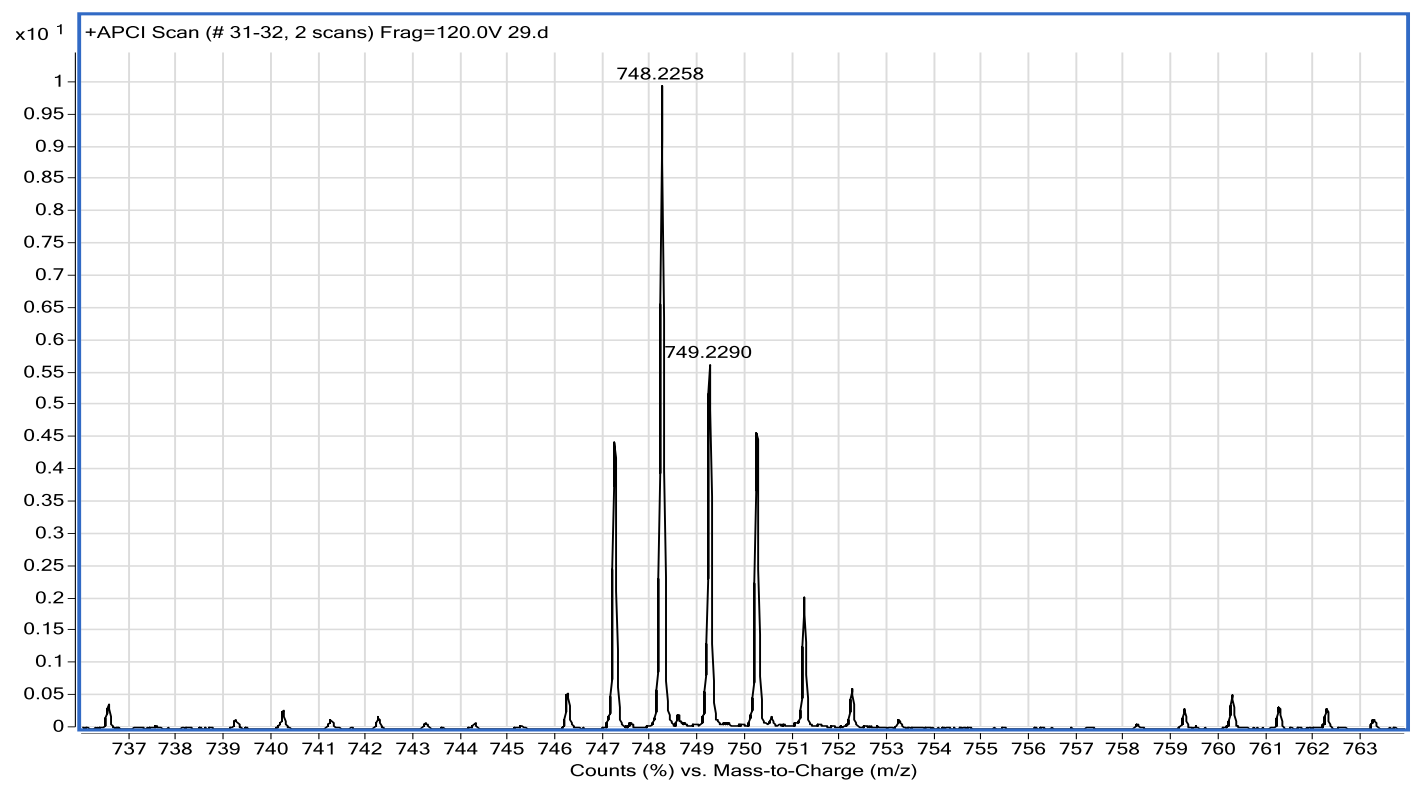

HRMS for FD5

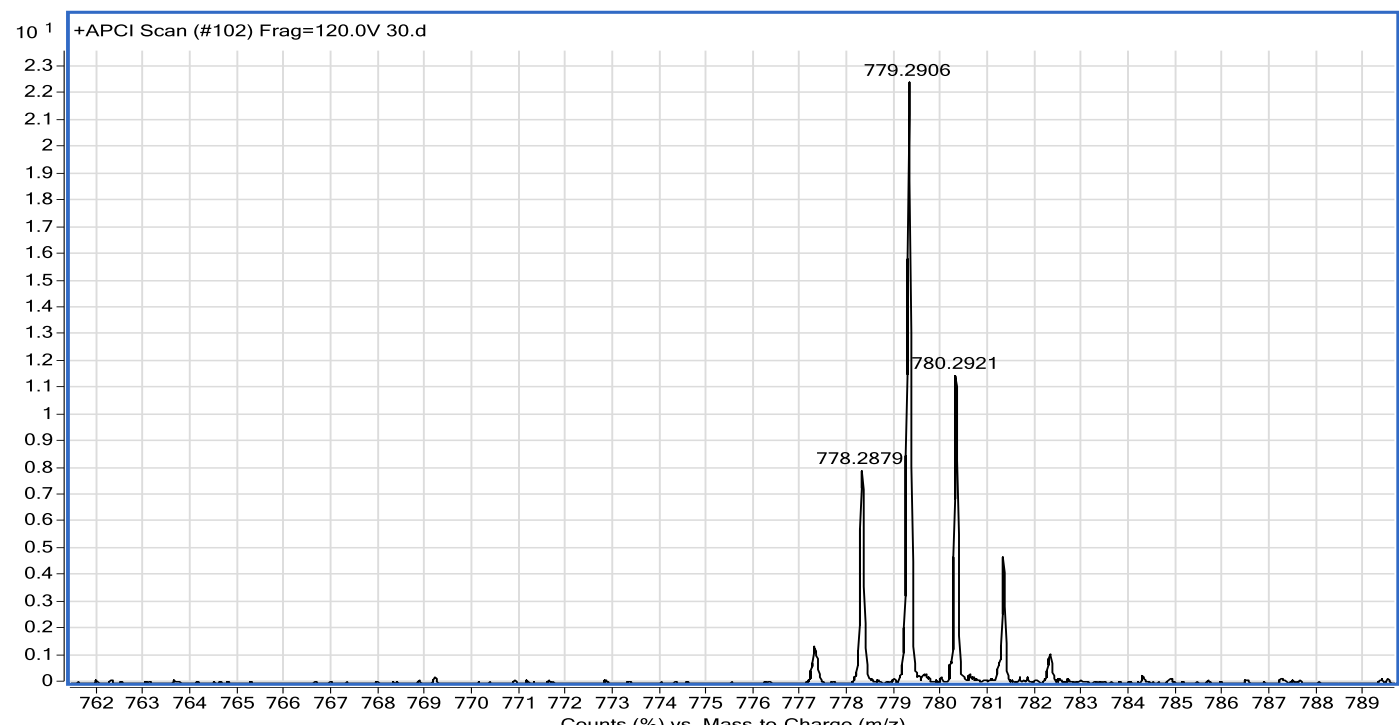

HRMS for FD6 


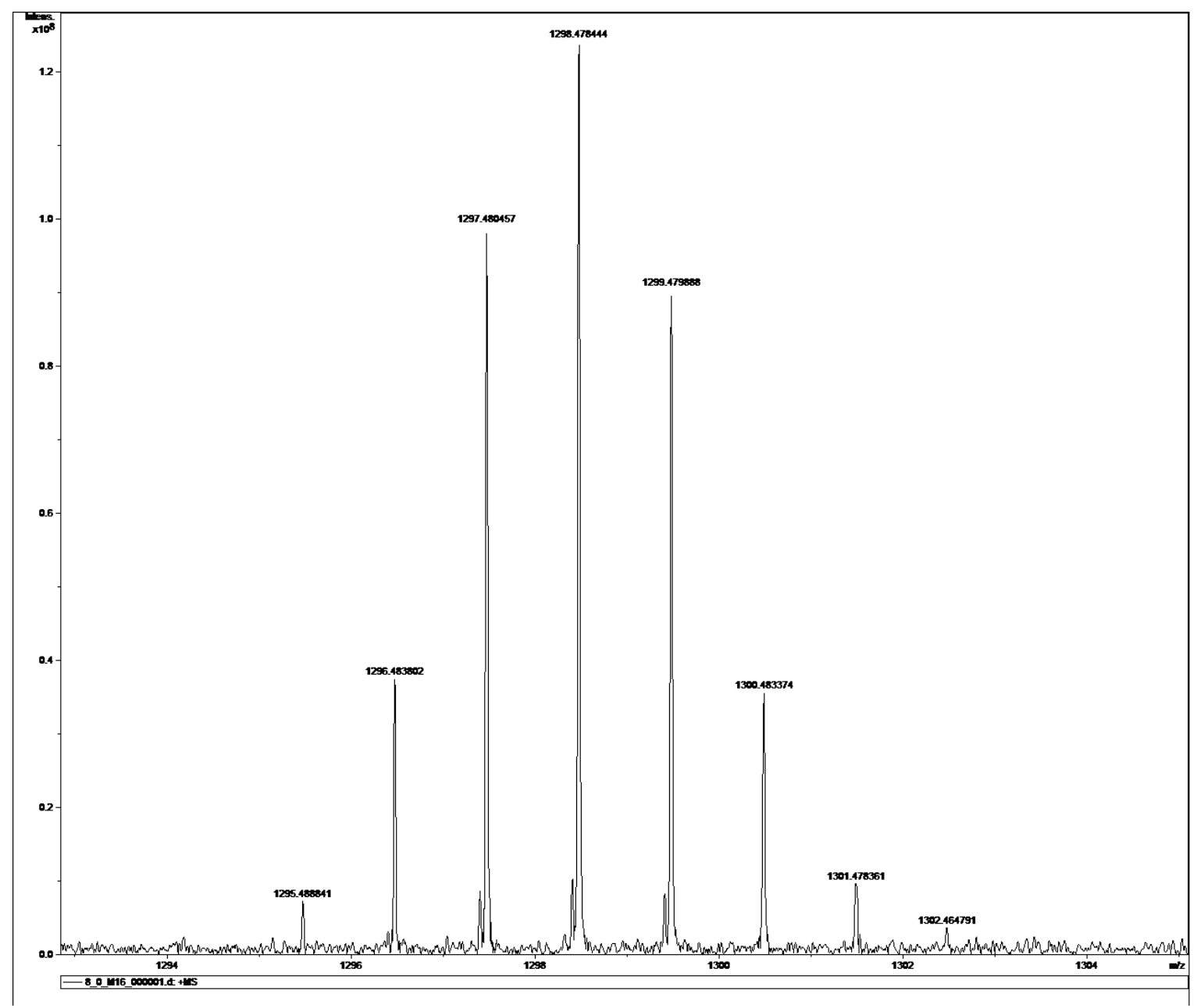

HRMS for T1 


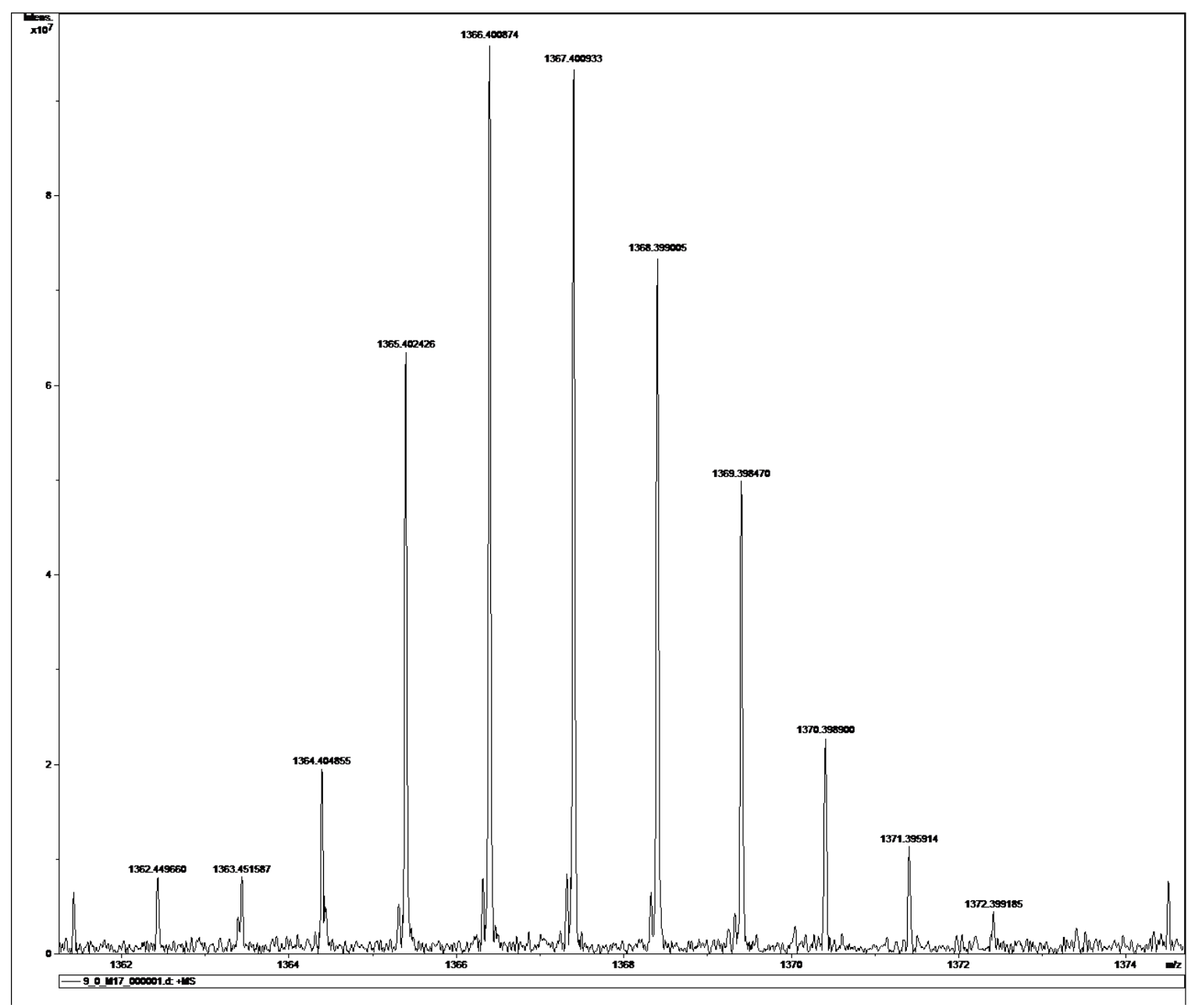

HRMS for T2 


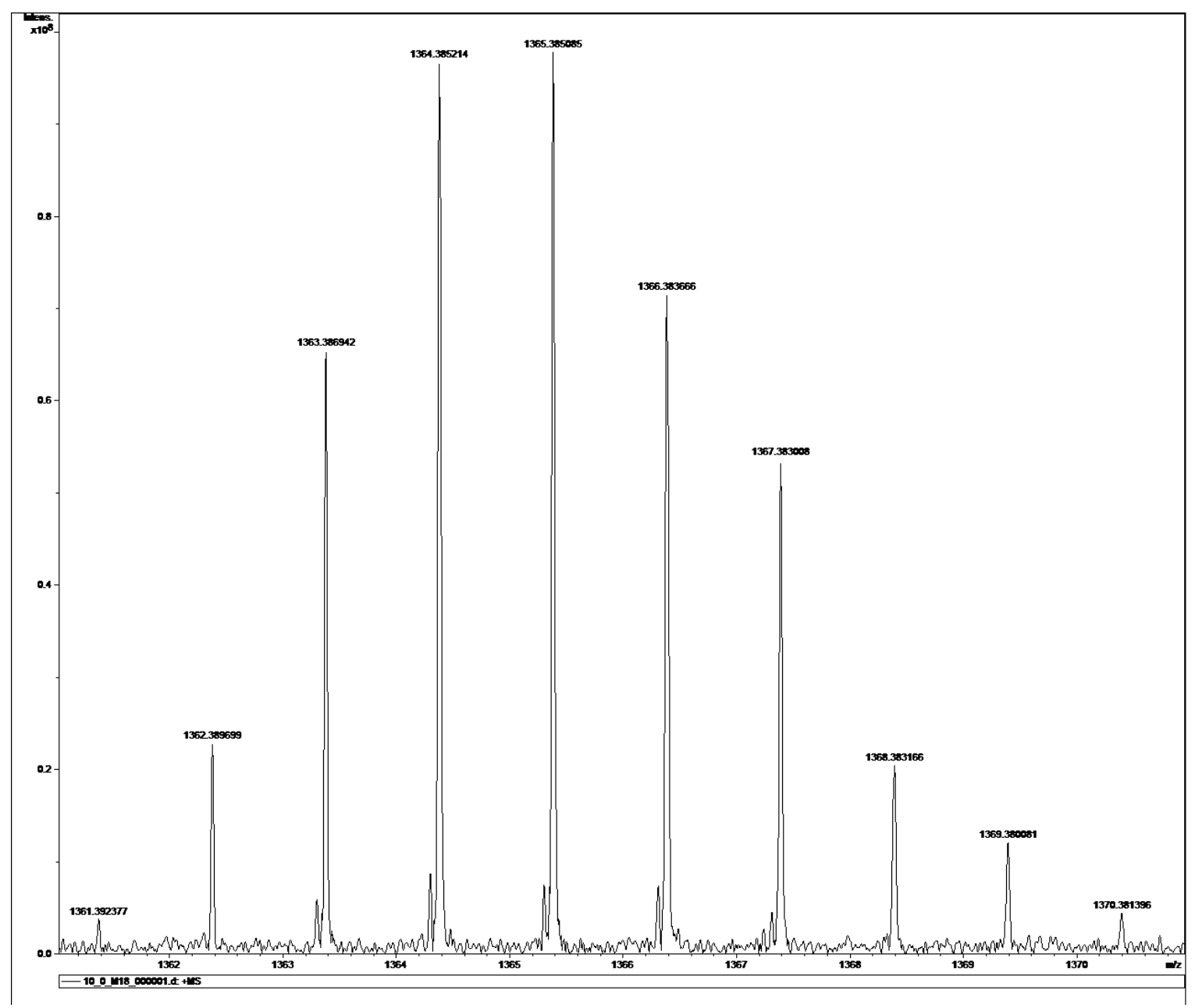

HRMS for T3 


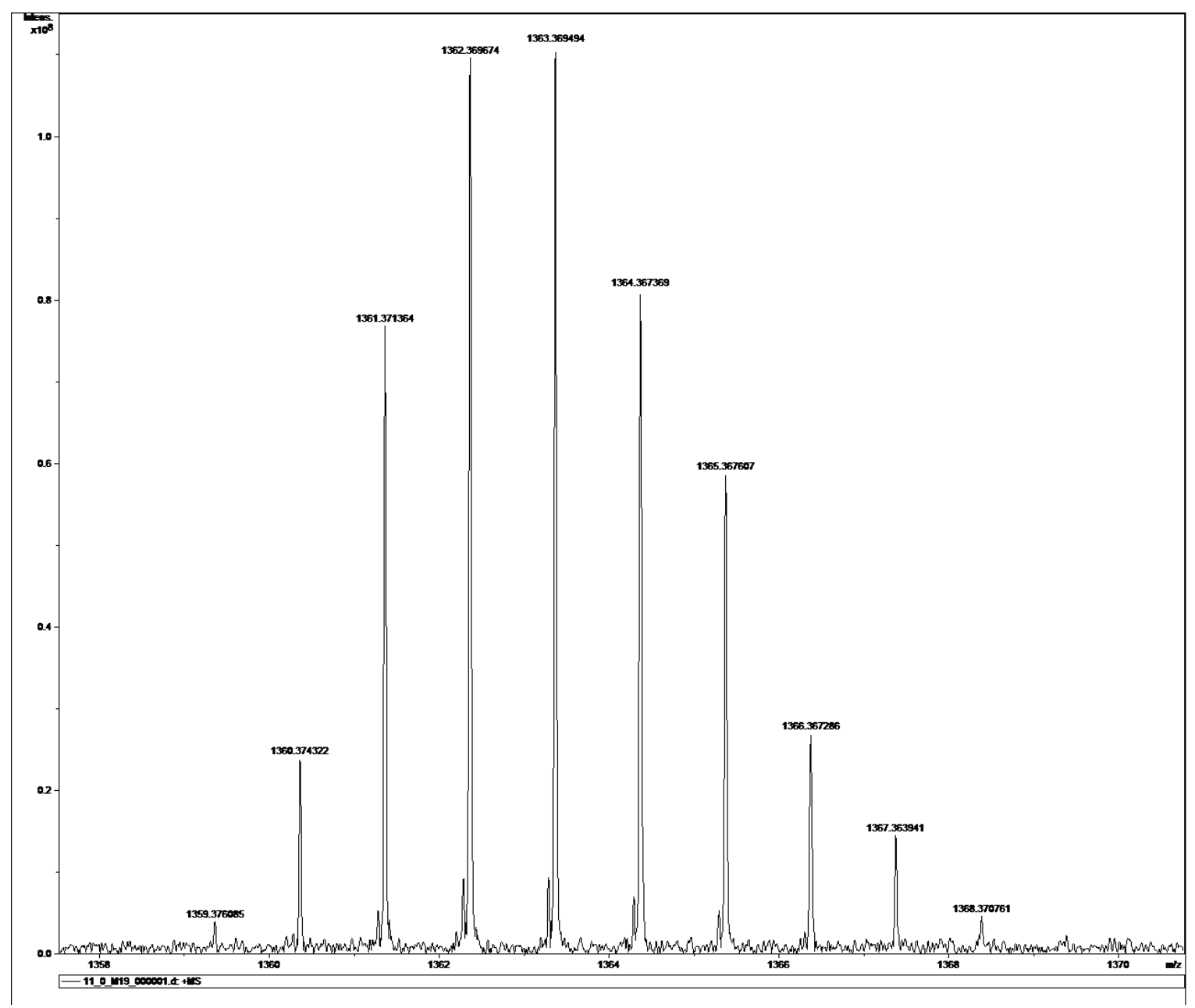

HRMS for T4 


\section{REFERENCES}

1) Isak, S.; Eyring, E. Fluorescence quantum yield of cresyl violet in methanol and water as a function of concentration. J. Phys. Chem. 1992, 96, 1738.

2) (a) Killoran, J.; Allen, L.; Gallagher, J. F.; Gallagher, W. M.; O'Shea. D. F. Synthesis of BF2 chelates of tetraarylazadipyrromethenes and evidence for their photodynamic therapeutic behavior. Chem. Commun. 2002, 1862. (b) Gorman, A.; Killoran, J.; O' Shea, C.; Kenna, T.; Gallagher, W. M.; O'Shea, D. F. In Vitro demonstration of the heavy-atom effect for photodynamic therapy. J. Am. Chem. Soc. 2004, 126, 10619.

3) Benson, R. C.; Kues, H. A. Fluorescence properties of indocyanine green as related to angiography. Phys. Med. Biol. 1978, 23, 159.

4) Software for the integration of CCD detector system Bruker analytical X-ray systems, Bruker axs, Madison, WI (after 2013).

5) APEX2 suite for crystallographic software, Bruker axs, Madison, WI.

6) Dolomanov, O.; BourhisL, J.; Gildea, R.; Howard, J.; Puschmann, H. Olex2: A complete structure solution, refinement and analysis program, J. Appl. Cryst, 2009, 42, 339.

7) Sheldrick, G.M. Crystal structure refinement with ShelXL, Acta Cryst, 2015, A71, 3.

8) Zhou, X.; Yu, C.; Feng, Z.; Yu, Y.; Wang, J.; Hao, E.; Wei, Y.; Mu, X.; Jiao, L. Highly regioselective $\alpha$-chlorination of the BODIPY chromophore with copper(II) chloride. Org. Lett. 2015, 17, 4632 .

9) Frisch, M. J.; Trucks, G. W.; Schlegel, H. B.; Scuseria, G. E.; Robb, M. A.; Cheeseman, J. R.; Scalmani, G.; Barone, V.; Mennucci, B.; Petersson, G. A.; Nakatsuji, H.; Caricato, M.; Li, X.; Hratchian, H. P.; Izmaylov, A. F.; Bloino, J.; Zheng, G.; Sonnenberg, J. L.; Hada, M.; Ehara, M.; Toyota, K.; Fukuda, R.; Hasegawa, J.; Ishida, M.; Nakajima, T.; Honda, Y.; Kitao, O.; Nakai, H.; Vreven, T.; Montgomery, J. A.; Peralta, J. E.; Ogliaro, F.; Bearpark, M.; Heyd, J. J.; Brothers, E.; Kudin, K. N.; Staroverov, V. N.; Keith, T.; Kobayashi, R.; Normand, J.; Raghavachari, K.; Rendell, A.; Burant, J. C.; Iyengar, S. S.; Tomasi, J.; Cossi, M.; Rega, N.; Millam, J. M.; Klene, M.; Knox, J. E.; Cross, J. B.; Bakken, V.; Adamo, C.; Jaramillo, J.; Gomperts, R.; Stratmann, R. E.; Yazyev, O.; Austin, A. J.; Cammi, R.; Pomelli, C.; Ochterski, J. W.; Martin, R. L.; Morokuma, K.; Zakrzewski, V. G.; Voth, G. A.; Salvador, P.; Dannenberg, J. J.; Dapprich, S.; Daniels, A. D.; Farkas, O.; Foresman, J. B.; Ortiz, J. V.; Cioslowski, J.; Fox, D. J. Gaussian 09, Revision D.01; Gaussian, Inc.: Wallingford, CT, 2013.

10) (a) Zong, L.; Zhang, H.; Li, Y.; Gong, Y.; Li, D.; Wang, J.; Wang, Z.; Xie, Y.; Han, M.; Peng, Q.; Li, X.; Dong, J.; Qian, J.; Li, Q.; Li, Z. Tunable aggregation-induced emission nanoparticles by varying isolation groups in perylene diimide derivatives and application in three-photon fluorescence bioimaging. ACS Nano, 2018, 12, 9532. (b) Zhang, Y.; Feng, L.; Wang, J.; Tao, D.; Liang, C.; Cheng, L.; Hao E.; Liu, Z. Surfactant-stripped micelles of near infrared dye and paclitaxel for photoacoustic imaging guided photothermal-chemotherapy. Small, 2018, 14, 1802991. 
11) (a) Ren, W.; Yan, Y.; Zeng, L.; Shi, Z.; Gong, A.; Schaaf, P.; Wang, D.; Zhao, J.; Zou, B.; Yu, H.; Chen, G.; Brown, E. M. B.; Wu, A. A near infrared light triggered hydrogenated black TiO2 for cancer photothermal therapy. Adv. Health. Mater., 2015, 4, 1526. (b) Liu, Y.; Ai, K.; Liu, J.; Deng, M.; He, Y.; Lu, L. Dopamine-melanin colloidal nanospheres: an efficient near-infrared photothermal therapeutic agent for in vivo cancer therapy. Adv. Mater., 2013, 25, 1353. 